\title{
18. PALYNOLOGY AND DINOFLAGELLATE BIOSTRATIGRAPHY OF DEEP SEA DRILLING PROJECT LEG 94, SITES 607 AND 611, NORTH ATLANTIC OCEAN1
}

\author{
Peta J. Mudie, Geological Survey of Canada, Atlantic Geoscience Centre, Dartmouth, N.S. ${ }^{2}$
}

\begin{abstract}
Cores from DSDP Holes $607,607 \mathrm{~A}, 611$, and $611 \mathrm{C}$ were sampled at about 1-m intervals for study of palynomorphs and palynodebris types. At both the subtropical Site 607 , west of the Azores $\left(41^{\circ} 07^{\prime} \mathrm{N}\right)$, and the high-latitude Gardar Drift Site $611\left(52^{\circ} 50^{\prime} \mathrm{N}\right)$, dinoflagellate cysts and pollen show cyclical variations in abundance, with peaks spaced 2 to $8 \mathrm{~m}$ apart downcore. This variation appears to correspond to climatic fluctuations with periods of 50,000 to 200,000 yrs. in the late Pliocene and Pleistocene, and to bottom sediment transport events or long-period climatic events during the late Miocene to early Pliocene. Well-preserved dinoflagellate cysts are present throughout the upper Miocene to Pleistocene sediments, but numbers of pollen and spores are too low to provide reliable biostratigraphic information.

Stratigraphic occurrences of dinoflagellate cysts were determined using 58 samples from Holes 607 and $607 \mathrm{~A}$ and 72 samples from Holes 611 and 611C. Lower upper Miocene (Subzone Ia), upper Miocene to lower Pliocene (Subzone Ib), lower to upper Pliocene (Zone II), upper Pliocene to middle Pleistocene (Subzone IIIa), and middle Pleistocene to Recent (Subzone IIIb) dinocyst zones were tentatively defined using ranges of diagnostic taxa present in European stratotypes and in DSDP reference sections; first occurrences of diagnostic taxa are not always synchronous, however, at the northern and southern sites. In the upper Miocene, Nematosphaeropsis sp. 1, N. oblonga n. sp., ?Cannosphaeropsis sp. 1, Operculodinium sp. 1, Pyxidiella sp. 1, and Impagidinium sp. A are important new taxa. In the lower to upper Pliocene, Impagidinium aliferum n. sp., Impagidinium sp. B., Impagidinium sp. C, Hystrichokolpoma sp. 1, and Impletosphaeridium spp. are useful guide fossils. The middle to upper Pleistocene is distinguished by several taxa with restricted ranges, including Multispinula sp. A, Glenodinium sp. A, and Piperodinium perplexum.
\end{abstract}

\section{INTRODUCTION}

On Leg 94 of the Deep Sea Drilling Project, upper Cenozoic sediments were drilled at six sites in the central North Atlantic, along a transect beginning in the subtropical waters west of the Azores and ending in the polar waters southeast of Greenland. Holes were continuously cored using the hydraulic piston corer (HPC) and extended core barrel (XCB) to obtain detailed records of the paleoceanographic and sedimentological histories of the sites. This chapter describes the dinoflagellate cysts and other palynomorphs from Site 607, near the south end of the transect, and from Site 611 at the north end of the transect. The site locations are shown in Figure 1; the salient site information is as follows:

Site 607, Holes 607 and $607 \mathrm{~A}$. Latitude $41^{\circ} 00.07^{\prime} \mathrm{N}$, longitude $32^{\circ} 57.44^{\prime} \mathrm{W}, 3427 \mathrm{~m}$ water depth, at the base of the upper western flank of the Mid-Atlantic Ridge, about $240 \mathrm{n}$. mi. northwest of the Azores.

Site 611 , Hole 611 . Latitude $52^{\circ} 50.47^{\prime} \mathrm{N}$, longitude $30^{\circ} 18.58^{\prime} \mathrm{W}, 3202.6 \mathrm{~m}$ water depth, at the crest of a sediment wave in the Gardar Drift on the southwestern flank of the Reykjanes Ridge.

Site 611 , Hole $611 \mathrm{C}$. Latitude $52^{\circ} 50.15^{\prime} \mathrm{N}$, longitude $30^{\circ} 18.10^{\prime} \mathrm{W}, 3227.6 \mathrm{~m}$ water depth, in the trough of a sediment wave about $0.75 \mathrm{n}$. mi. west of Hole 611 .

The objectives of the palynological studies proposed for Leg 94 included the following:

\footnotetext{
${ }^{1}$ Ruddiman, W. F., Kidd, R. B., Thomas, E., et al., Init. Repts. DSDP, 94: Washington (U.S. Govt. Printing Office).

Address: Geological Survey of Canada, Atlantic Geoscience Centre, P.O. Box 1006 , Dartmouth, N.S. B2Y 4A2, Canada.
}

1. To establish Neogene to Pleistocene dinoflagellate cyst zonations for subtropical Site 607 and subpolar Site 611.

2. To compare the dinoflagellate cyst stratigraphy from these holes with zonations established for other DSDP sites in the North Atlantic.

3. To compare the palynomorph and palynodebris characteristics of the northern and southern sites. The northern site presently lies in the path of strong westerly winds and has bottom topography dominated by large drift deposits; the southern site underlies the subtropical anticyclonic belt of variable winds, and here the bottom sediments are pelagic oozes draped over basement ridges.

\section{METHODS}

Palynological samples were taken at approximately $1-\mathrm{m}$ intervals. Sediment samples $\left(10-\mathrm{cm}^{3}\right.$ volume) representing 2-cm-long core intervals were disaggregated in $1 \%$ Calgon solution and sieved through screens with mesh openings of 150 and $10 \mu \mathrm{m}$. The coarse and fine fractions were saved for study of calcareous dinoflagellates. Two Eucalyptus tablets were added to the remaining sediment (10 to $150 \mu \mathrm{m}$ fraction) to provide a basis for estimating the palynomorph concentrations according to the method of Stockmarr (1971). These samples were chemically treated with $10 \% \mathrm{HCl}$, then with $52 \% \mathrm{HF}$. The organic residues were resuspended in Calgon and washed again on the $10-\mu \mathrm{m}$ screen to remove fine particulates that aggregate on the palynomorphs and obscure taxonomic features. The residues were finally stained with Safranin- 0 and mounted in glycerine gelatin.

This method resulted in adequate preparations for most of the samples. Many of the upper Pliocene and the Pleistocene samples, however, required further processing for removal of fine mineral debris. Repeated brief sonification, suspension in various detergents, and density separation in $\mathrm{ZnCl}$ improved the quality of these difficult samples; as noted by Manum (1976) and Mudie (1985), however, many of the preparations from sediments containing ice-rafted debris resisted all efforts to remove the clay-sized detritus. Oxidation with $\mathrm{HNO}_{3}$ also failed to remove pyrites and resulted in considerable loss of specimens. 


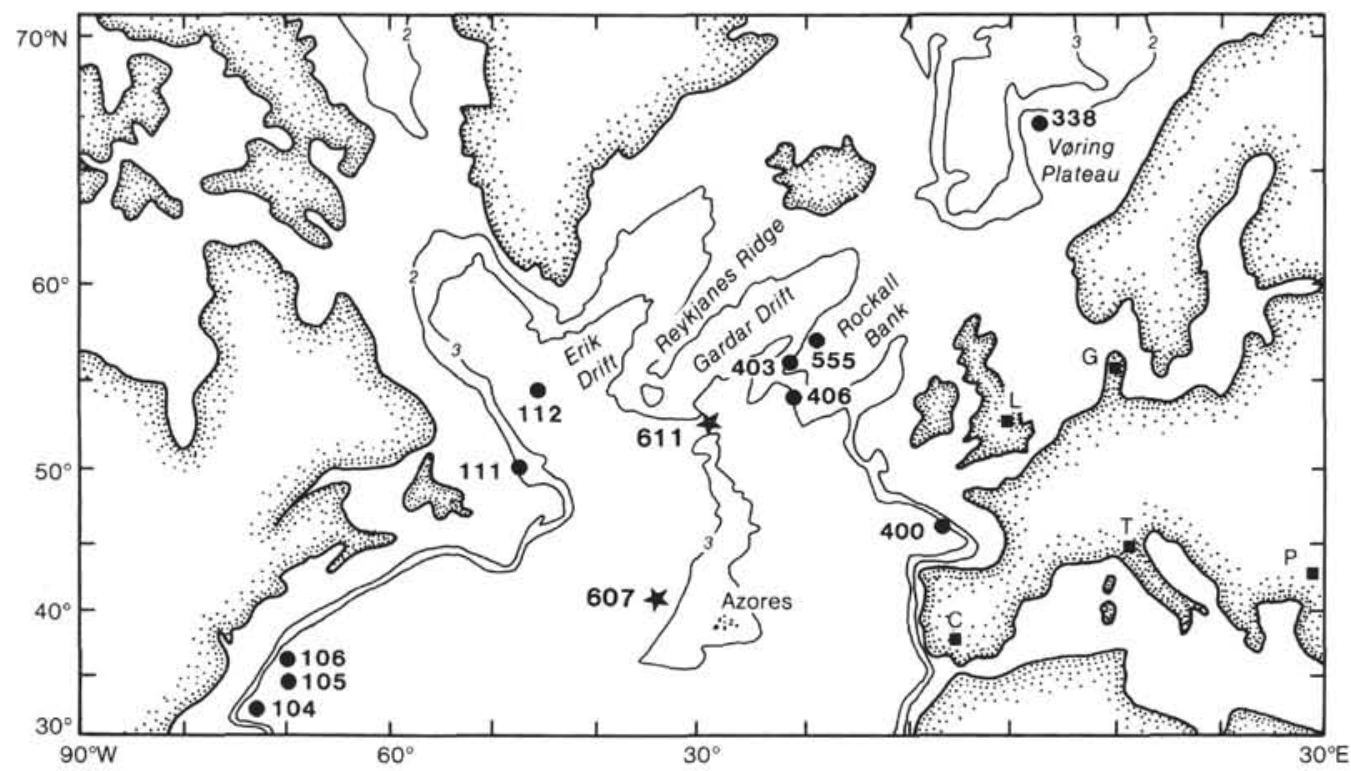

Figure 1. Map of the North Atlantic Ocean and surrounding continental regions, showing the locations of Leg 94 Sites 607 and 611, reference sections (squares), and other DSDP sites referred to in this chapter (large dots). Stratotype locations: $\mathrm{G}=$ Gram borehole; $\mathrm{C}=$ Carmona; $\mathrm{T}=$ Tabiano Bagni; $\mathrm{P}=$ Pannonian Basin; $\mathrm{L}=$ Ludham borehole. Bathymetric contours marked 2 and 3 indicate thousands of meters.

\begin{abstract}
Almost all of the palynological residues were very small and could be mounted on a single slide for examination with a Leitz Universal microscope and Orthoplan objectives. The entire content of each slide was first scanned at a magnification of $\times 160$ and all taxa were recorded. Taxonomic identifications were verified using magnifications of $\times 400$ and $\times 1000$. Subsequently, counts were made of traverses to obtain a total of up to 200 dinoflagellates.

The coarse fractions $(>150 \mu \mathrm{m})$ and very fine fractions $(<10 \mu \mathrm{m})$ from 10 samples of each hole were examined for calcareous dinoflagellates. The coarse fraction was dried and studied under a stereomicroscope. Smear slides of the fine sediment fraction $(<10 \mu \mathrm{m})$ were examined by transmitted-light microscopy. No calcareous dinoflagellates were found in any of these samples.
\end{abstract}

\section{RESULTS}

\section{Site 607, Holes 607 and 607A}

A total of 168 samples were examined from Holes 607 and 607A (Table 1). In Hole 607, 285 m of sediments were continuously cored: interbedded carbonate ooze and marls from 0 to $116 \mathrm{~m}$ sub-bottom depth, and foraminifer-nannofossil ooze from 117 to $285 \mathrm{~m}$. In Hole $607 \mathrm{~A}$ similar sediments were recovered to a depth of $311 \mathrm{~m}$, and samples from $282 \mathrm{~m}$ to the base of this hole were used to extend the palynostratigraphy. Details of the coring operations, lithology, and between-core correlation are reported by Ruddiman et al. (this volume).

Most samples from Site 607 contain well-preserved organic-walled palynomorphs; however, preservation was only moderately good at the base of Hole $607 \mathrm{~A}$ (Sections 26-2 and 26-3) and was moderate to poor in some samples from the upper Pliocene to lower Pleistocene intervals of Hole 607 (see Table 1). Dinoflagellate cysts were the dominant palynomorphs in all samples, with an average number of 195 cysts/g. Pollen and spores were less abundant, with an average of 44 grains/g. Small numbers of reworked Mesozoic to Paleogene palynomorphs (Table 1) occurred in many of the uppermost Pli- ocene and the Pleistocene samples. Rare reworked Paleogene dinocysts were found in many of the upper Miocene to lowermost Pliocene samples.

The main types of palynodebris (sensu Manum, 1976, and Habib, 1979) included (1) structureless amorphous organic debris (amorphogen of Bujak et al., 1977; see Plate 1, Fig. 1); (2) black (carbonized) organic fragments (Plate 1, Figs. 2 and 3); (3) pyritized diatoms (Plate 1, Figs. 11 and 13), silicoflagellates, and spherical algal spores (Plate 1, Fig. 9); (4) brown (lignified) wood fragments (Plate 1, Fig. 8); (5) translucent (nonlignified) plant fibers, tracheids (Plate 1, Fig. 6), and phytoliths; and (6) unicellular hyaline spores (Plate 1, Fig. 10). Other components of the palynological preparations (Table 1) are foraminiferal organic linings (Plate 1, Fig. 7), clay-sized mineral debris (Plate 1, Fig. 4), finely dispersed pyrite (Plate 1, Fig. 5), and volcanic glass, including translucent shards and black microtectites (Plate 1, Fig. 12).

Figure 2 shows that the average frequency of palynomorphs and palynodebris types tends to change systematically downhole at both Site 607 and Site 611 , thereby suggesting that the organic debris composition reflects regional rather than local changes in paleoenvironmental conditions and/or sediment sources. Figure 3, however, shows that the detailed graphs of selected components exhibit large-scale fluctuations in frequencies, with major peaks in dinoflagellate and pollen concentrations occurring together at roughly $10-\mathrm{m}$ intervals. Since the average sedimentation rate at Site 607 was about $44 \mathrm{~m} /$ m.y. (Site 607 report, this volume), these peaks correspond to time intervals of about 200,000 yrs., which lies within the range of periodicities of the eccentricity component of Milankovitch orbital cycles.

Fifty-eight of the most productive samples from Holes 607 and 607A were used to construct a tentative dinoflagellate biostratigraphy for Site 607 . Figure 4 shows the 
Table 1. Palynological data for samples (listed by section number) from Holes 607 and 607A.

\begin{tabular}{|c|c|c|c|c|c|c|c|c|c|c|c|c|c|c|c|c|c|c|}
\hline \multirow[b]{2}{*}{ Section } & Sub-bottom & & & & & & & Sp & & & & & & & & od & & \\
\hline & depth (m) & $\operatorname{Pr}$ & $\mathrm{Dr}$ & Dino & (S) & Pol & $\mathrm{Pt}$ & b & $\mathrm{t}$ & Diat & For & Amor & $\mathrm{Cl}$ & Coal & F & $\mathrm{Bn}$ & Py & M \\
\hline Hole 607 & & & & & & & & & & & & & & & & & & \\
\hline $1-1$ & 0.50 & & & 3423 & (15) & 290 & & & & & c & a & & & & & & \\
\hline $1-2$ & 2.00 & & $\mathrm{rP}$ & 74 & (16) & 2 & & & & & & & & & & & & \\
\hline $1-3$ & 3.50 & & & 294 & (4) & 25 & $\mathrm{r}$ & $\mathrm{r}$ & & & & f & $f$ & & & & & \\
\hline $1-4$ & 5.00 & & rD & 105 & (7) & 60 & & $\mathrm{r}$ & & & & c & & & & $\mathrm{r}$ & & \\
\hline $1-5$ & 6.50 & & & 102 & (7) & 16 & $\mathrm{r}$ & & & & & & a & c & & $\mathrm{r}$ & & \\
\hline $1-6$ & 8.00 & & & 86 & (7) & 20 & $\mathrm{r}$ & $\mathrm{r}$ & & & & f & c & $\mathrm{r}$ & & $\mathrm{r}$ & f & \\
\hline $2-1$ & 10.15 & & & 177 & (10) & 23 & & & & & & & & & & $r$ & & a \\
\hline $2-2$ & 11.60 & & $\mathrm{rP}$ & 285 & (21) & 85 & & f & & & & c & & & & & & $\mathrm{Vg}$ \\
\hline $2-3$ & 13.10 & & $\mathrm{aP} / \mathrm{rD}$ & 65 & (7) & 176 & & & & & & & & & & & & \\
\hline $2-4$ & 14.60 & & rD & 617 & (14) & 41 & & & & & a & a & a & & & r & & \\
\hline $2-5$ & 16.10 & & & 61 & (7) & 4 & & c & & & & & & & & & & \\
\hline $2-6$ & 17.60 & & & 1507 & (14) & 942 & & a & & & c & & a & & & & & \\
\hline $3-1$ & 19.70 & & $\mathrm{cP}$ & 55 & (4) & 69 & & & & & & va & va & c & & & & \\
\hline $3-2$ & 21.20 & & $\mathrm{cP}$ & 415 & (ii) & 101 & & $\mathrm{f}$ & & & & & & c & & c & & \\
\hline $3-3$ & 22.70 & & $\mathrm{rD}$ & 172 & (6) & 9 & & $\mathrm{r}$ & & & & c & & & $\mathrm{r}$ & c & & \\
\hline $3-4$ & 24.20 & & $\mathrm{rP}$ & 50 & (2) & 1 & & & & & & & & c & f & & & $\mathrm{Vg}$ \\
\hline $4-1$ & 25.70 & & $\mathrm{rP}$ & 111 & (8) & 32 & & & & & & & va & & & & & \\
\hline $4-2$ & 27.20 & & $\mathrm{rP}$ & 5 & (2) & 7 & & & & & & a & $r$ & f & & c & & \\
\hline $4-3$ & 28.70 & & fP & 1794 & (8) & 780 & & & & $f$ & & & va & a & & c & & $\mathrm{Vg}$ \\
\hline $4-4$ & 30.20 & & fP & 86 & (6) & 21 & & & & & & a & va & a & & & & \\
\hline $4-5$ & 31.70 & & $\mathrm{rP}$ & 29 & (5) & 0 & & $\mathrm{r}$ & & & & s & & & I & & & $\mathrm{Vg}$ \\
\hline $4-6$ & 33.20 & & & 0 & 0 & 0 & & & & & a & & & & & f & & \\
\hline $5-1$ & 35.30 & & & 0 & 0 & 0 & & & & & a & & & & & & & \\
\hline $5-2$ & 36.80 & & & 12 & (5) & 0 & & $r$ & & $\mathrm{r}$ & c & c & a & & a & a & a & \\
\hline $5-3$ & 38.30 & & & 1450 & (10) & 450 & & & & & f & a & a & & $\mathrm{r}$ & & & \\
\hline $5-4$ & 39.80 & & & 295 & (12) & 20 & & c & & & & & a & & $\mathrm{r}$ & & & \\
\hline $5-5$ & 41.30 & & & 0 & 0 & 0 & & & & & & & a & & & & & \\
\hline $5-6$ & 42.80 & & & 208 & (14) & 22 & & c & & & & a & & & $\mathrm{r}$ & & & \\
\hline $6-1$ & 44.90 & & & 214 & (8) & 31 & & & & & & f & & a & & & a & $\mathrm{Vg}$ \\
\hline $6-2$ & 46.40 & & & 299 & (10) & 6 & & $r$ & & $\mathrm{r}$ & & & a & a & $\mathrm{r}$ & & & \\
\hline $6-3$ & 47.90 & & & 92 & (4) & 1 & & f & $\mathrm{r}$ & $\mathrm{r}$ & a & & a & & & & & a \\
\hline 6-4 & 49.40 & & & 379 & (9) & 76 & & f & & $\mathrm{r}$ & & c & & c & $\mathrm{r}$ & $\mathrm{r}$ & & \\
\hline $6-5$ & 50.90 & & & 782 & (12) & 63 & & c & & & & & & a & & $\mathrm{r}$ & & a \\
\hline $6-6$ & 52.40 & & & 1300 & (7) & 79 & & & & & & & a & & & & & \\
\hline $7-1$ & 54.50 & M & & 130 & (2) & 0 & & & & & & & & & & $r$ & a & \\
\hline $7-2$ & 56.00 & & & 261 & (13) & 48 & & & & & & & a & $f$ & & & & a \\
\hline $7-3$ & 57.50 & & $\mathrm{rP}$ & 673 & (15) & 194 & & $\mathrm{r}$ & & & & a & a & & $r$ & & & \\
\hline $7-4$ & 59.00 & & & 528 & (10) & 0 & & & & $r$ & & & & & f & & & $\mathrm{Vg}$ \\
\hline $7-5$ & 60.50 & & & 1753 & (8) & 0 & & & & & & & a & & & & & va \\
\hline $7-6$ & 62.00 & & & 21 & (2) & 0 & & & & & & & a & c & & $r$ & & $a$ \\
\hline $8-1$ & 64.10 & & & 339 & (14) & 169 & & & & & & & va & & & c & & $\mathrm{Vg}$ \\
\hline $8-2$ & 65.60 & MP & & 223 & (6) & 20 & & & & & & & & & r & & a & a \\
\hline $8-3$ & 67.10 & & & 115 & (11) & 8 & & c & & & & & & a & $r$ & $\mathrm{r}$ & & c \\
\hline $8-4$ & 68.60 & & & 54 & (6) & 20 & & $\mathrm{r}$ & & & & $r$ & & & f & $\mathrm{r}$ & & \\
\hline $8-5$ & 70.10 & & rA & 244 & (14) & 11 & & & & $r$ & & & va & & c & $\mathrm{r}$ & & \\
\hline $8-6$ & 71.60 & & & 322 & (6) & 46 & & & & & & & & & & & a & a \\
\hline $9-1$ & 73.70 & & & 106 & (6) & 12 & $r$ & a & $r$ & & & $\mathrm{r}$ & & & c & $\mathrm{r}$ & a & \\
\hline $9-2$ & 75.20 & & $\mathrm{fD} / \mathrm{rA}$ & 60 & (10) & 2 & & c & $r$ & & & c & c & & f & & & $\mathrm{Vg}$ \\
\hline $9-3$ & 76.70 & & & 191 & (11) & 39 & & c & & & & f & a & & f & & & \\
\hline $9-4$ & 78.20 & & rD & 34 & (2) & 5 & $r$ & & & & & f & & & f & c & & \\
\hline $9-5$ & 79.70 & & & 0 & 0 & 0 & & & & & & & a & & & & & \\
\hline $9-6$ & 81.20 & & & 0 & (3) & 0 & & & & & & & a & & & & & \\
\hline $10-1$ & 83.30 & & & 53 & (6) & 12 & & & c & $r$ & & c & & & c & $\mathrm{r}$ & a & \\
\hline $10-2$ & 84.80 & & & 22 & (4) & 4 & & & & & & $\mathrm{r}$ & & & c & & & \\
\hline $10-3$ & 86.30 & & & 357 & (12) & 27 & & c & & & & & va & & $\mathrm{r}$ & & & \\
\hline $10-4$ & 87.80 & & & 215 & (3) & 18 & & & & & & f & & & & & & c \\
\hline $10-5$ & 89.10 & & $\mathrm{rP} / \mathrm{D}$ & 96 & (4) & 123 & & c & & & & a & & & & & & \\
\hline $10-6$ & 90.80 & & $\mathrm{cP} / \mathrm{D}$ & 307 & (23) & 51 & & & & & & & & c & c & & & $\mathrm{Vg}$ \\
\hline $11-1$ & 92.90 & & & 90 & (11) & 15 & & & & & & a & a & & c & & & \\
\hline $11-2$ & 94.40 & & & 88 & (7) & 81 & $r$ & & & & & & a & & c & & & $\mathrm{Vg}$ \\
\hline $11-3$ & 95.90 & & & 157 & (7) & 7 & & & & & & & a & a & c & & & a \\
\hline $11-4$ & 97.40 & & & 18 & (2) & 0 & & & & & & & a & a & f & & a & a \\
\hline $11-5$ & 98.90 & & & 54 & (11) & 5 & & f & & & & & c & & f & & & \\
\hline $11-6$ & 100.40 & & $\mathrm{rP}$ & 82 & (5) & 0 & & & & & & & & c & & & $\mathrm{a}$ & $\mathrm{Vg}$ \\
\hline $12-1$ & 102.5 & & & 75 & (12) & 32 & & & & $r$ & & & & & I & & va & \\
\hline $12-2$ & 104.00 & & & 4 & (3) & 0 & f & & & & & f & & & f & & & \\
\hline $12-3$ & 105.50 & & $\mathrm{rD}$ & 696 & (18) & 164 & & f & & & & & a & & & & & \\
\hline $12-4$ & 107.00 & & & 38 & (2) & 8 & & a & & $\mathrm{r}$ & & & & a & c & $\mathrm{r}$ & a & \\
\hline $12-5$ & 108.50 & & & 0 & 0 & 2 & & $\mathrm{r}$ & & & & & a & & & & & $\mathrm{Vg}$ \\
\hline $12-6$ & 110.00 & & $\mathrm{rP}$ & 113 & (12) & 5 & $r$ & & f & a & & & & a & c & & a & \\
\hline $13-1$ & 112.10 & & & 0 & 0 & 0 & & $r$ & & & & & c & & & & & \\
\hline $13-2$ & 113.60 & & & 0 & 0 & 0 & & & & & & & c & & & & & \\
\hline $13-3$ & 115.10 & & & 0 & 0 & 0 & & & & & & & c & & & & & \\
\hline $13-4$ & 116.60 & & & 0 & 0 & 0 & & & & & & & c & & & & & \\
\hline $13-5$ & 118.10 & & & 90 & (3) & 0 & & & & & & & a & & f & & & $\mathrm{Vg}$ \\
\hline
\end{tabular}


Table 1 (continued).

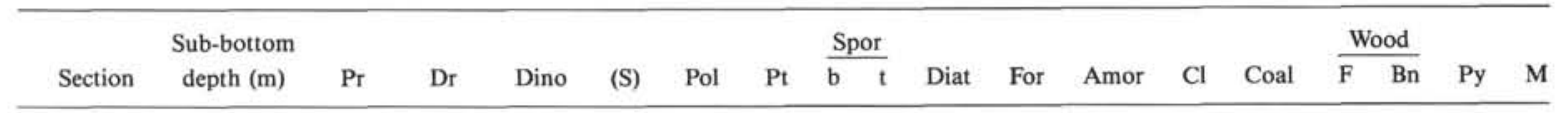

Hole 607 (Cont.)

\begin{tabular}{|c|c|c|c|c|c|c|c|c|c|c|c|c|c|c|c|c|c|c|}
\hline $13-6$ & 119.60 & & & 153 & (3) & 0 & & & & & & & $\mathrm{a}$ & & & & & $\mathrm{Vg}$ \\
\hline $14-1$ & 121.70 & & & 24 & (3) & 0 & $r$ & $\mathbf{a}$ & & & & & va & $\mathrm{a}$ & a & & $\mathrm{a}$ & \\
\hline $14-2$ & 123.20 & & & 203 & (12) & 0 & & & & $\mathrm{r}$ & & & $\mathrm{a}$ & $\mathrm{c}$ & c & & & $\mathrm{a}$ \\
\hline $14-3$ & 124.70 & & & 144 & (8) & 0 & & & & & & & $\mathrm{a}$ & a & c & & a & $\mathrm{Vg}$ \\
\hline $14-4$ & 126.20 & & & 234 & (9) & 12 & & & & & & & a & & f & & & \\
\hline $14-5$ & 127.70 & & $\mathrm{rP}$ & 55 & (13) & 4 & & a & & $\mathrm{c}$ & & & a & & c & & a & a \\
\hline $14-6$ & 129.20 & & & 45 & (5) & 22 & & & & & & $r$ & & & f & & & \\
\hline $15-1$ & 131.30 & & & 182 & (6) & 52 & $r$ & & & $\mathrm{r}$ & $r$ & & & c & & & a & a \\
\hline $15-2$ & 132.80 & & & 124 & (4) & 0 & & $\mathrm{f}$ & & & & r & & & & & & $\mathrm{Vg}$ \\
\hline $15-4$ & 135.80 & & & 78 & (5) & 0 & & & & & & f & & c & $\mathrm{r}$ & & a & a \\
\hline $16-1$ & 140.90 & & D & 161 & (13) & 27 & & & & & & $r$ & & $\mathrm{c}$ & $\mathrm{r}$ & & $\mathrm{a}$ & $\mathrm{Vg}$ \\
\hline $16-2$ & 142.40 & & & 116 & (6) & 0 & & & & & & c & & & $r$ & & & \\
\hline $16-3$ & 143.90 & & $\mathrm{rP}$ & 205 & (9) & 0 & & & & & & c & & & f & & a & a \\
\hline $18-1$ & 160.10 & $\mathrm{P}$ & & 59 & (4) & 0 & & & & & & f & & a & $\mathrm{r}$ & & a & \\
\hline $18-2$ & 161.60 & & & 163 & (5) & 0 & & & & & & f & & $\mathrm{a}$ & $\mathrm{r}$ & & $\mathbf{a}$ & Vg \\
\hline $18-3$ & 163.10 & & & 29 & (2) & 14 & $r$ & & & & & c & & & f & & a & \\
\hline $18-4$ & 164.60 & & & 44 & (3) & 0 & & $r$ & & & & & & & r & & $a$ & \\
\hline $18-5$ & 166.10 & & & 14 & (4) & 14 & & $\mathrm{r}$ & & & & $\mathrm{c}$ & & & $\mathrm{r}$ & & $\mathrm{c}$ & \\
\hline $18-6$ & 167.60 & & $\mathrm{rD}$ & 42 & (6) & 13 & & & $r$ & & & f & & & $\mathrm{r}$ & & $\mathrm{c}$ & $\mathrm{Vg}$ \\
\hline $19-1$ & 170.00 & & & 14 & (1) & 0 & & & & & $\mathrm{r}$ & & & & & & $\mathrm{a}$ & a \\
\hline $19-3$ & 172.70 & & & 25 & (4) & 25 & & & $\mathrm{r}$ & & & $\mathrm{c}$ & & a & c & & $\mathrm{a}$ & $\mathrm{Vg}$ \\
\hline $19-4$ & 174.20 & & & 51 & (5) & 7 & & & & & & & & & c & & a & $a$ \\
\hline $19-5$ & 175.70 & & $\mathrm{rD}$ & 7 & (5) & 0 & & & & & & a & & & c & $r$ & a & \\
\hline $19-6$ & 177.20 & & & 33 & (7) & 22 & & & & & & a & & c & c & & a & \\
\hline $20-1$ & 179.30 & & & 13 & (2) & 4 & & & & & & $r$ & & & c & & c & \\
\hline $20-2$ & 180.80 & & $\mathrm{rD}$ & 23 & (4) & 0 & $\mathrm{r}$ & & & & & f & & & & & $\mathrm{c}$ & \\
\hline $20-3$ & 182.30 & & & 16 & (2) & 0 & & & & & & & & & $r$ & & a & $\mathrm{a}$ \\
\hline $20-4$ & 183.80 & & & 40 & (6) & 13 & & & & & & c & & & c & & a & \\
\hline $20-5$ & 185.30 & & $\mathrm{rD}$ & 0 & 0 & 0 & & & & & & & & & & & & Vg \\
\hline $20-6$ & 186.80 & & & 45 & (7) & 0 & $r$ & $\mathrm{r}$ & & & & $\mathrm{c}$ & & $\mathrm{r}$ & f & $r$ & f & \\
\hline $21-1$ & 188.90 & & & 58 & (4) & 19 & & & & & & f & & $\mathrm{r}$ & f & & $\mathrm{a}$ & \\
\hline $21-2$ & 190.40 & & & 86 & (13) & 22 & & & & & & $\mathrm{c}$ & & & f & $r$ & $\mathrm{c}$ & $\mathrm{Vg}$ \\
\hline $21-3$ & 191.90 & & $\mathrm{rD}$ & 44 & (10) & 33 & $\mathrm{f}$ & c & & & & c & & c & & & a & \\
\hline $21-4$ & 193.40 & & & 81 & (9) & 7 & & & & & & $r$ & & $\mathrm{r}$ & f & & a & a \\
\hline $21-5$ & 194.90 & & & 59 & (11) & 8 & & a & & & & & & & $\mathrm{c}$ & & a & Vg \\
\hline $21-6$ & 196.40 & & & 99 & (8) & 40 & $r$ & & & & & f & & c & f & & a & \\
\hline $22-1$ & 198.50 & & & 22 & (2) & 33 & $\mathrm{r}$ & & & $r$ & & f & & $\mathrm{r}$ & f & & $\mathrm{c}$ & \\
\hline $22-2$ & 200.00 & & & 94 & (10) & 4 & & & & & & $r$ & & & & & & \\
\hline $22-3$ & 201.50 & & & 119 & (14) & 6 & $r$ & $r$ & & & & $r$ & & & f & & & \\
\hline $22-4$ & 203.00 & & & 62 & (8) & 7 & & a & & & & c & & & r & $r$ & a & \\
\hline $22-5$ & 204.50 & & & 32 & (4) & 6 & & $\mathrm{r}$ & & & & c & c & c & f & & a & \\
\hline $22-6$ & 206.00 & MP & $\mathrm{rD}$ & 2600 & (8) & 371 & & va & & & & f & & c & $\mathrm{f}$ & & a & \\
\hline $23-1$ & 208.10 & & & 192 & (17) & 8 & & $a$ & & & & a & & & 0 & & $a$ & Vg \\
\hline $23-2$ & 209.60 & & & 8 & (4) & 0 & & c & & & & $\mathrm{f}$ & & & c & & & $\mathrm{Vg}$ \\
\hline $23-3$ & 211.10 & & & 50 & (5) & 8 & & a & & & & & $\mathrm{a}$ & a & r & & & \\
\hline $23-4$ & 212.60 & & & 93 & (7) & 9 & & a & & & & c & & a & c & & & $\mathrm{Vg}$ \\
\hline $23-5$ & 214.10 & & & 27 & (3) & 0 & $r$ & & & $r$ & & c & & & a & $\mathrm{r}$ & $\mathrm{c}$ & \\
\hline $23-6$ & 215.60 & & & 44 & (17) & 2 & $r$ & & & & & & & & c & & & \\
\hline 24-1 & 217.70 & & & 12 & (3) & 12 & & & & & & va & & & a & & va & $\mathrm{Vg}$ \\
\hline $24-2$ & 219.20 & & & 38 & (5) & 0 & & $f$ & & & & $\mathrm{c}$ & & a & a & & c & $\mathrm{Vg}$ \\
\hline $24-3$ & 220.70 & & & 72 & (4) & 14 & & & & $r$ & & $r$ & & c & I & & & $\mathrm{Vg}$ \\
\hline $24-4$ & 222.20 & & & 57 & (5) & 10 & & & & $r$ & & $r$ & & & c & & & $\mathrm{Vg}$ \\
\hline $24-5$ & 223.70 & & & 1067 & (11) & 133 & & $\mathrm{a}$ & c & & & a & & & a & & a & a \\
\hline $24-6$ & 225.20 & & & 190 & (7) & 21 & $\mathrm{r}$ & $\mathrm{r}$ & & & & r & & & & & & \\
\hline $25-1$ & 227.30 & & $r P$ & 24 & (3) & 0 & & & f & & & $\mathrm{f}$ & & & c & & & \\
\hline $25-2$ & 228.80 & & & 45 & (4) & 0 & & c & & & $\mathbf{r}$ & c & & & $\mathrm{r}$ & & c & \\
\hline 26-1 & 236.90 & & & 27 & (4) & 0 & & & & & & a & & & $c$ & & & \\
\hline $26-2$ & 238.40 & & & 69 & (3) & 46 & & a & & r & & & & $\mathrm{c}$ & c & & & \\
\hline $26-3$ & 239.90 & & & 90 & (12) & 0 & & c & & & & c & & c & & & c & \\
\hline $26-4$ & 241.40 & & & 37 & (4) & 0 & & & & & & $\mathrm{c}$ & & a & f & & c & \\
\hline $27-1$ & 246.50 & & & 147 & (17) & 20 & & $r$ & & & & $\mathrm{c}$ & & & r & & a & \\
\hline $27-2$ & 248.00 & & & 373 & (10) & 13 & & va & & & & a & & & $\mathrm{c}$ & & a & \\
\hline $27-3$ & 249.50 & & & 364 & (12) & 18 & & $a$ & & & & va & & va & $r$ & & va & \\
\hline $27-4$ & 251.00 & & & 223 & (13) & 0 & & $\mathrm{a}$ & & & & & & & & & & \\
\hline $28-6$ & 264.00 & & & 232 & (9) & 15 & & va & & & & a & & a & $\mathrm{f}$ & & a & \\
\hline $29-2$ & 267.30 & & & 330 & (12) & 14 & $\mathrm{r}$ & $\mathrm{a}$ & $r$ & & & & & a & & & a & \\
\hline $29-3$ & 268.80 & & & 52 & (20) & 2 & & $\mathrm{a}$ & & c & & & a & & $r$ & & a & $\mathrm{Vg}$ \\
\hline $30-1$ & 275.34 & & $\mathrm{rP}$ & 87 & (14) & 5 & r & & & & & c & & & c & & & \\
\hline $30-2$ & 276.84 & & & 84 & (6) & 0 & & $\mathrm{c}$ & & & & & $r$ & & I & & $\mathrm{r}$ & \\
\hline $30-3$ & 278.34 & & & 61 & (11) & 0 & f & $f$ & & & & & $\mathrm{c}$ & $\mathrm{a}$ & c & & c & \\
\hline
\end{tabular}

Hole 607A 
Table 1 (continued).

\begin{tabular}{|c|c|c|c|c|c|c|c|c|c|c|c|c|c|c|c|c|c|c|}
\hline \multirow[b]{2}{*}{ Section } & \multirow{2}{*}{$\begin{array}{l}\text { Sub-bottom } \\
\text { depth (m) }\end{array}$} & \multirow[b]{2}{*}{$\operatorname{Pr}$} & \multirow[b]{2}{*}{ Dr } & \multirow[b]{2}{*}{ Dino } & \multirow[b]{2}{*}{ (S) } & \multirow[b]{2}{*}{ Pol } & \multirow[b]{2}{*}{$\mathrm{Pt}$} & \multicolumn{2}{|c|}{$\underline{\text { Spor }}$} & \multirow[b]{2}{*}{ Diat } & \multirow[b]{2}{*}{ For } & \multirow[b]{2}{*}{ Amor } & \multirow[b]{2}{*}{$\mathrm{Cl}$} & \multirow[b]{2}{*}{ Coal } & \multicolumn{2}{|c|}{ Wood } & \multirow[b]{2}{*}{ Py } & \multirow[b]{2}{*}{ M } \\
\hline & & & & & & & & b & $\mathrm{t}$ & & & & & & $\mathrm{F}$ & $\mathrm{Bn}$ & & \\
\hline \multicolumn{19}{|c|}{ Hole 607A (Cont.) } \\
\hline $24-3$ & 286.00 & & & 149 & (14) & 25 & & $f$ & & & & a & & & & & a & \\
\hline $24-5$ & 289.00 & & & 85 & (8) & 0 & & a & $r$ & & & r & & f & c & & & VG \\
\hline $24-6$ & 290.50 & & & 59 & (5) & 0 & & c & & & & $r$ & & a & c & & & \\
\hline $25-1$ & 292.60 & & & 0 & 0 & 0 & & & & & & & & & & & & $\mathrm{Vg}$ \\
\hline $25-2$ & 294.10 & & & 188 & (9) & 0 & & & & r & & $\mathrm{r}$ & & & & & & \\
\hline $25-3$ & 295.60 & & & 116 & (6) & 0 & & & & & & a & & & c & & a & \\
\hline $25-4$ & 297.10 & & & 133 & (5) & 44 & & $\mathrm{c}$ & & $\mathrm{r}$ & & $r$ & & & c & & & \\
\hline $25-6$ & 300.10 & & & 64 & (4) & 0 & & & & $\mathrm{r}$ & & c & & c & a & & a & \\
\hline $26-1$ & 302.20 & & & 30 & (3) & 10 & $r$ & & & & & c & & & va & & a & \\
\hline $26-2$ & 303.70 & & & 71 & (15) & 2 & $\mathrm{r}$ & f & & $\mathrm{r}$ & & & a & & a & & a & \\
\hline $26-3$ & 305.20 & & & 197 & (22) & 10 & & f & & & & & & & & & & a \\
\hline
\end{tabular}

Note: $\mathrm{Pr}=$ preservation state $(\mathrm{M}=$ moderate, $\mathrm{P}=$ poor, unspecified $=$ good $) . \mathrm{Dr}=$ relative abundance of reworked palynomorphs $(\mathrm{P}=$ pollen and spores; $\mathrm{D}=$ dinocysts; $\mathrm{A}=$ acritarchs); Dino = number of dinocysts $/ \mathrm{g} ;(\mathrm{S})=$ dinocysts species diversity; Pol = number of pollen and spores/g. Relative abundances: phytoliths $(\mathrm{Pt})$; algal spores (Spor) of the spherical black type (b) and the translucent type (t); pyritized diatom fragments (Diat); foraminifers (For); amorphogen (Amor); clay flocs $(\mathrm{Cl})$; carbonized organic fragments $(\mathrm{Coal})$; wood particles $(\mathrm{F}=\mathrm{nonligni}$ fied fibers; $\mathrm{Bn}=$ brown lignified fragments); pyrite $(\mathrm{Py})$; and mineral particles $(\mathrm{M})$, including volcanic glass $(\mathrm{Vg})$. Relative abundance scale: $\mathrm{r}=$ rare $(<5 \%) ; f=$ frequent $(5-15 \%) ; c=$ common $(>15-30 \%) ; a=$ abundant $(>30-50 \%)$; va $=$ very abundant $(>50 \%)$.

distributions of the 56 recognized taxa and their ranges relative to the chronostratigraphy reported for Site 607 (Site 607 report, this volume, fig. 6). The most common taxa throughout the holes are Nematosphaeropsis labyrinthea, Impagidinium aculeatum, I. paradoxum, I. patulum, Spiniferites ramosus, S. mirabilis, Tectatodinium pellitum, and Operculodinium centrocarpum, most of which have long-ranging (Paleogene or middle Miocene to Recent) distributions in the North Atlantic. Three informal dinocyst concurrent-range zones and two subzones can be delimited, however, on the basis of the concurrent ranges of certain species which have a first appearance datum (FAD) or last appearance datum (LAD) in Neogene or Pleistocene stratotypes or in other timeequivalent reference sections.

Dinocyst Zone I at Site 607 is distinguished primarily by the presence of Achomosphaera andalousiense, which is commonly associated with $A$. ramulifera, Tectatodinium simplex, Invertocysta spp. (I. lacrymosa and I. tabulata), Impagidinium velorum, Operculodinium crassum, ?O. echigoense, and $O$. wallii. Also common in this zone are several small species of Impagidinium (Impagidium sp. A and sp. B), Operculodinium sp. of Jan du Chêne (1977), Operculodinium sp. of Piasecki (1980), and Lingulodinium machaerophorum. Several less common species have distributions that are confined to or extend just above the boundary between dinocyst Zones I and II: Nematosphaeropsis oblonga, N. aquaeducta, Incertae sedis sp. I of Edwards (1984), and ?Operculodinium sp. 1.

Dinocysts Zone I is tentatively subdivided on the basis of several species that make their first appearances in Sample 607A-24-1, 49-51 cm. The base of Subzone 1b at Site 607 is marked by the first occurrences of $\mathrm{Amicu}$ losphaera umbracula, Nematosphaeropsis sp. 1, and Platycystidia(?) sp. 1 of Manum (1976). The following species have first occurrences just above the Subzone $1 \mathrm{a} / 1 \mathrm{~b}$ boundary: Spiniferites mirabilis, Corrudinium harlandii, Operculodinium longispinigerum, ?Cannosphaeropsis sp. 1, and Pyxidiella sp. 1. The FADs of Operculodi- nium centrocarpum, O. crassum, and Palaeostomocystis spp. of Baltes (1971) occur in the middle to upper part of Subzone 1b.

The base of Hole 607A (and nominal base of dinocyst Subzone 1a) has an early late Miocene (Tortonian) age of about 7.1 Ma, as determined by paleomagnetic data and microfossil zonations reported in the Site 607 chapter (this volume). The boundary between Subzones $\mathrm{la}$ and $\mathrm{lb}$ lies just above the boundary between the NeOgloboquadrina humerosa and Globorotalia conomiozea planktonic foraminiferal zones, and has an approximate age of $6.5 \mathrm{Ma}$, on the basis of magnetostratigraphic data from Site 607. The top of Subzone $1 \mathrm{~b}$ is placed at Sample 607-23-6, 49-51 cm. This boundary corresponds to the LADs of the following species: Achomosphaera andalousiense, Nematosphaeropsis sp. 1, Platycystidia(?) sp. of Manum (1976), Pyxidiella sp. 1, and ?Cannosphaeropsis sp. 1. The boundary at Site 607 corresponds closely to the top of the coccolith Zone NN13, and it lies within the $\mathrm{C} 2$ chronozone, which has an age of 4.57 to $4.77 \mathrm{Ma}$ (Clement and Robinson, this volume). Hence, dinocyst Subzone 1b at Site 607 spans the Miocene/Pliocene boundary and includes the Messinian and lower Zanclean (Tabianian) stages of the Mediterranean stratotypes. It is also noteworthy that the LADs of $L$. machaerophorum, $O$. wallii, and ?O. echigoense occur at the Miocene/Pliocene boundary (top of the Messinian), in Sample 607-27-2, 49-51 cm.

Dinocyst Zone II at Site 607 is distinguished primarily by the presence of Amiculosphaera umbracula, Achomosphaera ramulifera, and Corrudinium harlandii, all of which have their LADs at the boundary between Zones II and III, in Sample 607-10-6, 49-51 cm. These species frequently occur together with Spiniferites splendidus and Impagidium aliferum, which have their FADs just above the Zone I/II boundary and which disappear just below the Zone II/III boundary. The FAD of Impagidinium japonicum also occurs just above the Zone I/II boundary, in Sample 607-23-6, 49-51 cm, and the LAD of this species occurs just above the Zone II/III boundary. Oth- 

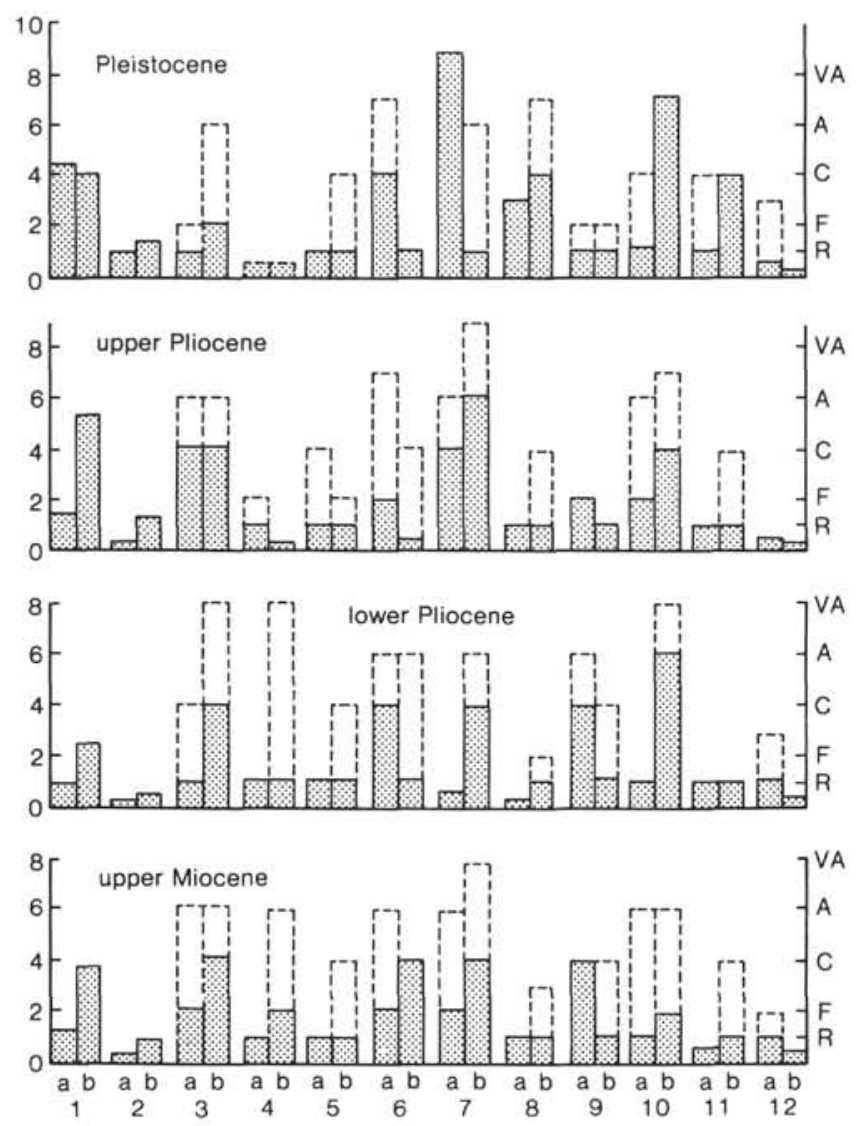

Figure 2. Bar graphs showing average abundance and maximum amount (dashed line) of palynomorphs and palynodebris types at Site 607 (bar a) and Site 611 (bar b) for the Pleistocene and Neogene. The following numbers identify the bar pairs: 1 . Number of dinocysts/g $\times 10^{2} .2$. Number of pollen, fern, and moss spores $/ \mathrm{g} \times 10^{2} .3-12$. Relative abundances of (3) black algal spores, (4) translucent spores, (5) pyritized diatoms, (6) amorphogen, (7) clay flocs, (8) lignified wood fragments, (9) nonlignified fibers and phytoliths, (10) carbonized organic fragments, (11) reworked palynomorphs, and (12) volcanic glass. Vertical scale at left indicates number of palynomorphs per gram $\times 10^{2}$. Vertical scale at right indicates visual abundance, where $\mathrm{R}=\operatorname{rare}(<5 \%) ; \mathrm{F}=$ frequent $(5-15 \%) ; \mathrm{C}=$ common $(>15-30 \%) ; \mathrm{A}=$ abundant $(>30-50 \%) ; \mathrm{VA}=$ very abundant $(>50 \%)$.

er common species in Zone II are Invertocysta spp. (mostly I. lacrymosa), Operculodinium sp. of Jan du Chêne, and Operculodinium sp. of Piasecki (1980), all of which have their LADs just below the top of Zone II. Labyrinthodinium truncatum, Operculodinium crassum, and $O$. longispinigerum all have last occurrences near the middle of Zone II, at or just above the lower/upper Pliocene boundary. At Site 607, the following species have their LADs in the lower part of Zone II: Nematosphaeropsis oblonga, Incertae sedis sp. I of Edwards (1984), Tectatodinium simplex, Operculodinium crassum, and Palaeostomocystis spp. of Baltes (1971). Several taxa have their FADs in the upper part of Zone II: Polysphaeridium zoharyi, Spiniferites bentori, and $S$. cf. pseudofurcatus, Selenopemphix nephroides, Operculodinium psilatum, and Brigantedinium spp.

Dinocyst Zone II in Hole 607 has an age corresponding to the interval extending from the top of NN13 in the lowermost Pliocene to the middle of NN18 and the planktonic foraminiferal Zone PL6 in the upper Pliocene. This places the age of the Zone II/III boundary between $1.90 \mathrm{Ma}$ (top of NN18) and $2.20 \mathrm{Ma}$ (base of PL6) at Site 607. The boundary between the lower and upper Pliocene (3.4 Ma) in Zone II of Site 607 is notably marked by the LAD of Operculodium longispinigerum and the FAD of Polysphaeridium zoharyi. It is also notable that the top of the Gauss chronozone ( 2.5 $\mathrm{Ma}$ ) in Hole 607 corresponds closely to the LADs of two common late Miocene taxa, Operculodinium sp. of Jan du Chêne (1977) and Operculodinium sp. of Piasecki (1980), and to the FAD of the peridinioid genera Brigantedinium and Selenopemphix.

Dinocyst Zone III at Site 607 is notable for its relatively low diversity of species, which are dominated by Impagidinium aculeatum, I. patulum, Tectatodinium pellitum, Spiniferites ramosus, S. mirabilis, and Brigantedinium spp. The FADs of Operculodinium psilatum, $\mathrm{Fi}$ lisphaera filifera, Impagidinium strialatum, and Spiniferites membranaceus occur at or near the base of Zone III, in Sections 607-10-6, 607-10-5, 607-9-3, 607-9-2, respectively. Zone III of Site 607 is tentatively subdivided on the basis of the extinction of several long-ranging Neogene species in the lower Pleistocene interval and the restricted occurrence of four late Pleistocene genera in the upper Pleistocene. Subzone IIIa is marked by the LADs of Impagidinium japonicum, Impagidinium sp. $\mathrm{B}$, and Impagidinium sp. C. Filisphaera filifera has a restricted range in Subzone IIIa, and its LAD marks the boundary between Subzones IIIa and IIIb in Sample $607-6-5,49-51 \mathrm{~cm}$. The following species have FADs at or near the middle of Zone IIIa: Impagidinium strialatum (Section 607-9-3), Operculodinium israelianum (Section 607-8-6), Brigantedinium auranteum, and Zygabikodinium lenticulatum (Section 607-7-5).

The base of Subzone IIIb in Hole 607 is marked by the first occurrences of Ataxiodinium choanum and Piperodinium perplexum in Sample 607-6-4, 49-51 cm and the FAD of Brigantedinium simplex in Sample 607-5-3, $49-51 \mathrm{~cm}$. The FAD of Votadinium calvum is in Sample 607-2-6, 49-51 cm. Multispinula minuta and Glenodinium sp. A are restricted to the top of Subzone IIIb in Section 607-1-1.

Subzone IIIa in Hole 607 extends from the middle of Zone NN18 to the middle of Zone NN19, which is correlated to the Jaramillo Chron. The Jaramillo Chron spans the time interval from 0.98 to $0.91 \mathrm{Ma}$, and its base marks the age of the top of the Calabrian Stage. Thus, dinocyst Zone IIIa in Hole 607 spans the Pliocene/Pleistocene boundary and includes the Calabrian Stage. It is notable that the range of Filisphaera filifera corresponds closely to the Calabrian Stage (1.7-0.98 Ma) in Hole 607. Zone IIIb in Hole 607 corresponds to the top of the Matuyama and the entire Bruhnes Chronozones.

\section{Site 611, Holes 611 and 611C}

A total of 219 samples were examined from Holes 611 and $611 \mathrm{C}$ (Table 2). In Hole $611,125.8 \mathrm{~m}$ of marly calcareous nannofossil oozes, alternating with calcareous or terrigenous muds, were continuously cored. In 

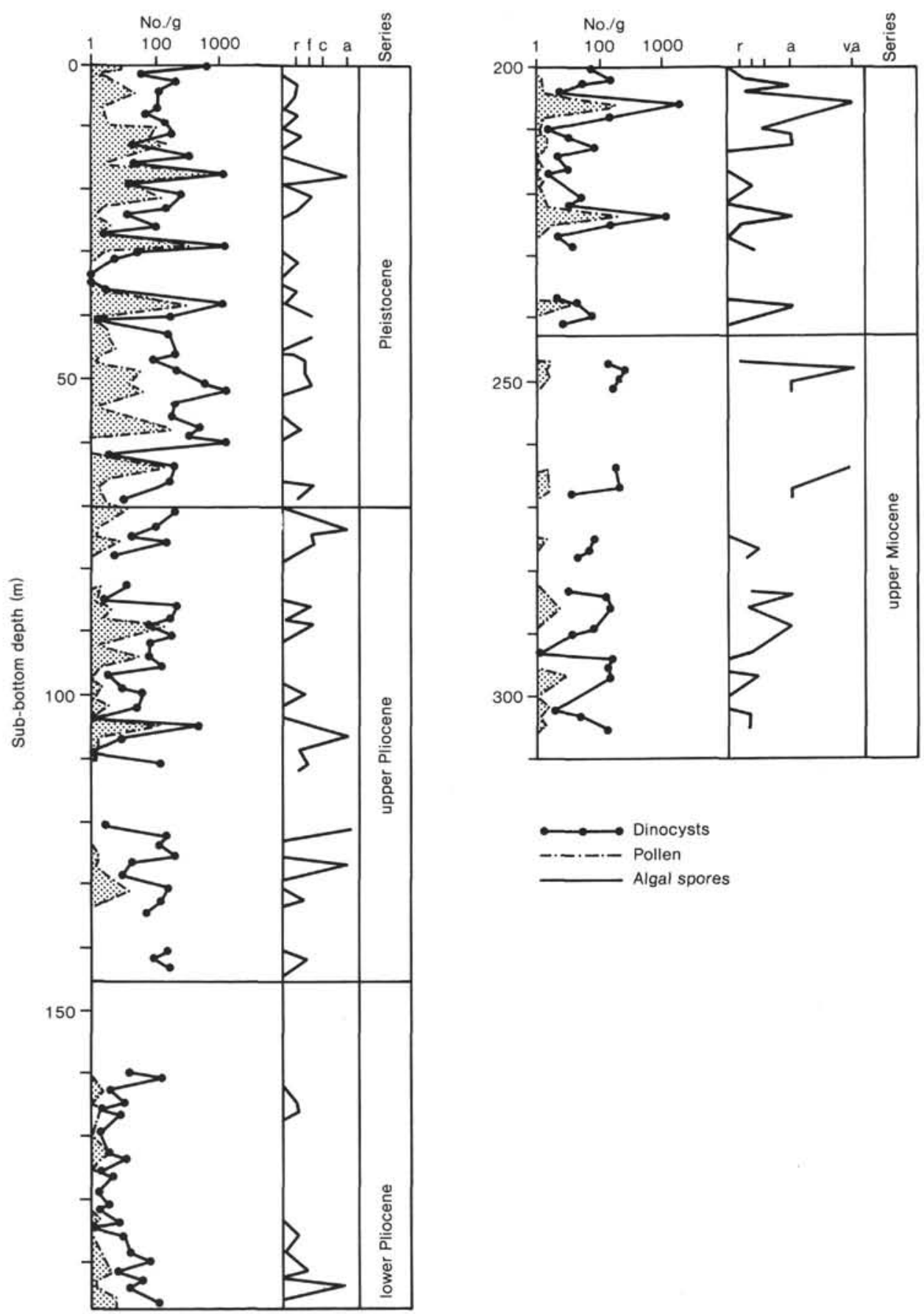

Figure 3. Dinocyst and pollen concentrations (number per gram) and relative abundances of algal spores plotted against depth and series in Holes 607 and 607A. Note that the palynomorph abundance scale is logarithmic; relative abundances are $\mathrm{r}=\operatorname{rare}(<5 \%) ; \mathrm{f}=$ frequent $(5-15 \%) ; \mathrm{c}=$ common $(>15-$ $30 \%) ; \mathrm{a}=$ abundant $(>30-50 \%) ; \mathrm{va}=$ very abundant $(>50 \%)$.

Hole $611 \mathrm{C}$, similar sediments were cored to a depth of $114 \mathrm{~m}$, followed downsection by siliceous-calcareous oozes and calcareous mud to $149 \mathrm{~m}$, then siliceous calcareous oozes and calcareous oozes to $451 \mathrm{~m}$, and nannofossil chalk with marly intervals to the base at $511.6 \mathrm{~m}$. Palynological samples from Hole 611 were examined down to $124.19 \mathrm{~m}$ (Section 611-14-6), and samples from $124.49 \mathrm{~m}$ (Section 611C-15-5) to $510.09 \mathrm{~m}$ (Section 611C47-6) from Hole $611 \mathrm{C}$ were used for this report.
As at Site 607, most samples from Site 611 contained well-preserved organic-walled palynomorphs (Table 2), although several intervals of moderate to poor preservation were found in the Pleistocene sediments and a few intervals of moderate to poor preservation were scattered through the lower Pliocene and Miocene sediments. The frequency of dinocysts (average number $=367 / \mathrm{g}$ ) at Site 611 was consistently higher than at Site 607, except in the Pleistocene interval (see Fig. 2). Likewise, the 


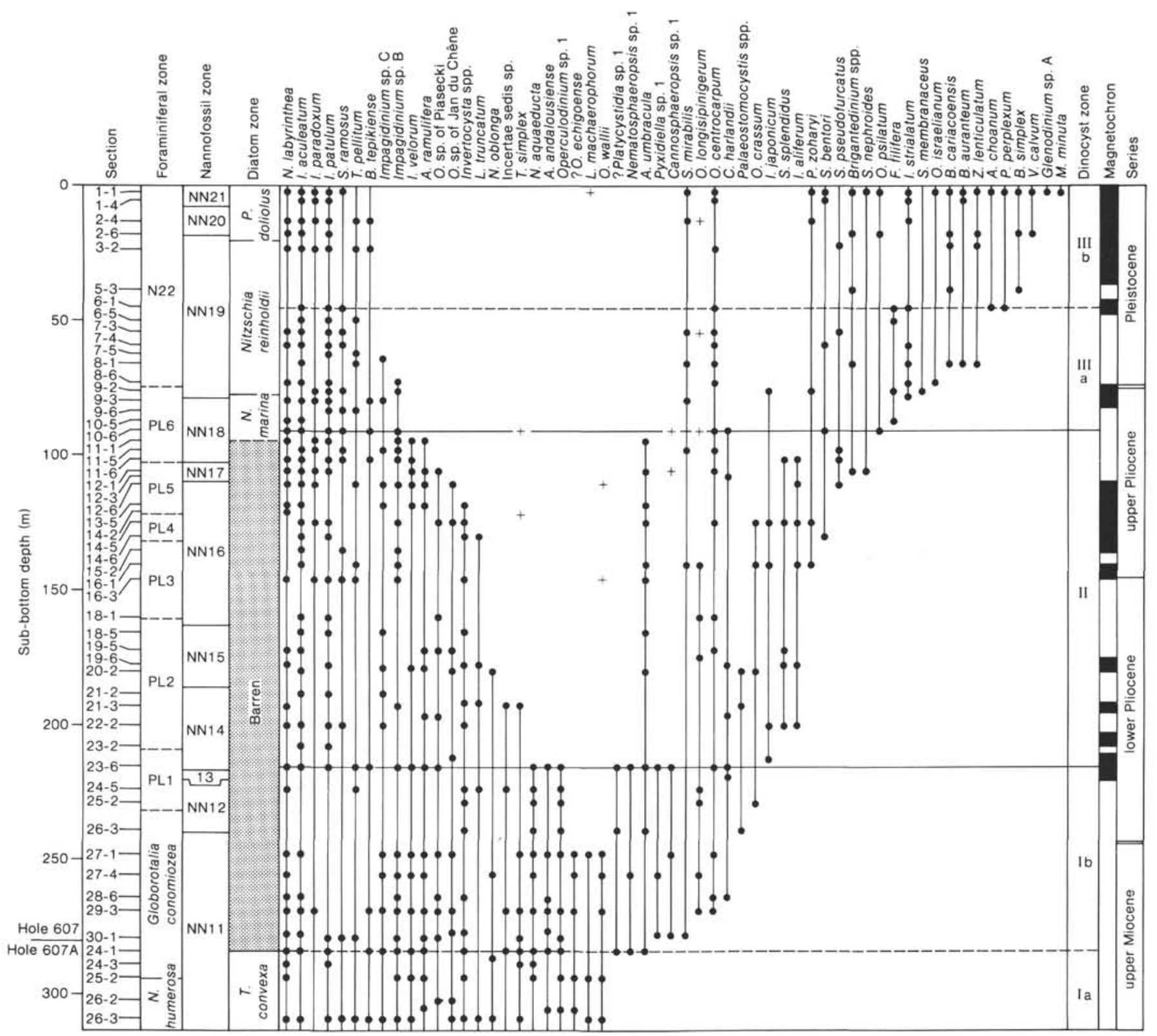

Figure 4. Range and distribution chart of dinocysts recovered from Neogene to Pleistocene sediments in Holes 607 and $607 \mathrm{~A}$. Dots indicate presence of well-preserved specimens; + indicates rare, poorly preserved specimens, probably reworked. Foraminiferal, calcareous nannofossil, and diatom zones, and magnetostratigraphy and age determinations, are from Leg 94 site reports (this volume). Magnetochrons: shaded = normal polarity; unshaded $=$ reversed polarity.

frequency of pollen and spores (average number $=83$ / g) was mostly much higher at Site 611 . In addition, the number of reworked Mesozoic to Paleogene pollen, dinoflagellates, and acritarchs was consistently higher throughout the upper Pliocene and the Pleistocene of Site 611, and reworked Paleogene dinoflagellates were more common in the Miocene and lower Pliocene (see Table 2).

The types of palynodebris found at Site 611 were the same as those described earlier for Site 607 (Plate 1). Figure 2 illustrates the salient differences between the dominant categories at the two sites: carbonized organic fragments and clay-impregnated organic flocs were generally common to abundant throughout Holes 611 and $611 \mathrm{C}$, and lignified wood fragments were more frequent- ly present than at Site 607 . Algal spores (both black and translucent types) and peaks of pyritized siliceous microfossils were generally more common in the upper Miocene/lower Pliocene intervals. In contrast, amorphogen and plant fibers tended to be less abundant throughout the pre-Pleistocene sediments at Site 611 .

The detailed graphs of palynomorph frequencies for Site 611 (Fig. 5) show that relatively large-amplitude fluctuations in dinoflagellate, pollen, and spore frequencies generally persist throughout the Pliocene and upper Miocene intervals at Site 611. Futhermore, regular oscillations of pollen and spore frequencies persist to the base of Hole 611C (late Miocene, NN9; Site 611 report, this volume). Data in Table 2 also show that there is a consis- 
Table 2. Palynological data for samples (listed by section number) from Holes 611 and $611 \mathrm{C}$.

\begin{tabular}{|c|c|c|c|c|c|c|c|c|c|c|c|c|c|c|c|c|c|c|}
\hline & Sub-bottom & & & & & & & $\mathrm{Sr}$ & & & & & & & & od & & \\
\hline Section & depth $(m)$ & Pr & Dr & Dino & (S) & Pol & $\mathrm{P} t$ & $b$ & 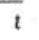 & Diat & For & Amor & $\mathrm{Cl}$ & Coal & $\mathrm{F}$ & $\mathrm{Bn}$ & Py & M \\
\hline Hole 611 & & & & & & & & & & & & & & & & & & \\
\hline $1-1$ & 0.49 & & $\mathrm{cP}$ & 394 & (9) & 139 & & & & & & & & $\mathrm{a}$ & $\mathrm{r}$ & c & & \\
\hline $2-1$ & 1.59 & P & $\mathrm{cP} / \mathrm{D}$ & 234 & (7) & 426 & & & & & & & & a & & & & $\mathrm{a}$ \\
\hline $2-3$ & 4.49 & & $\mathrm{cP}, \mathrm{fD}$ & 0 & 0 & 0 & & & & & & & & a & & a & & a \\
\hline $2-4$ & 5.99 & & $\mathrm{rA}$ & 5 & (2) & 0 & & & c & & & a & & & $\mathrm{r}$ & & f & a \\
\hline $2-5$ & 7.49 & & $\mathrm{rP} / \mathrm{D}$ & 495 & (8) & 151 & & a & & & & c & & & c & $\mathrm{r}$ & & \\
\hline $2-6$ & 8.99 & $\mathrm{P}$ & $\mathrm{cP}, \mathrm{rD}$ & 96 & (6) & 113 & & c & $\mathrm{r}$ & & & & & a & & $\mathrm{a}$ & & \\
\hline $3-1$ & 11.09 & & $\mathrm{rP} / \mathrm{D}$ & 0 & 0 & 29 & & & c & & $\mathrm{r}$ & & & $f$ & $\mathrm{r}$ & c & & \\
\hline $3-2$ & 12.59 & M & $\mathrm{cP}, \mathrm{rD}, \mathrm{A}$ & 67 & (5) & 84 & & & $\mathrm{r}$ & & & & & $\mathrm{a}$ & & c & & \\
\hline $3-3$ & 14.09 & & $\mathrm{rP}$ & 65 & (4) & 0 & & & & & & & & & c & $r$ & & \\
\hline $3-4$ & 15.59 & & $\mathrm{rP}$ & 8 & (1) & 16 & & f & & & & & & a & $\mathrm{r}$ & c & & a \\
\hline $3-5$ & 17.09 & & & 144 & (2) & 0 & & & & & & & & a & & $\mathrm{f}$ & & a \\
\hline $3-6$ & 18.59 & & $\mathrm{rP} / \mathrm{D}$ & 625 & (4) & 20 & & & & & & & & a & & & & a \\
\hline $4-1$ & 20.69 & & & 0 & 0 & 0 & & $\mathrm{r}$ & & & & & & a & & & & va \\
\hline $4-2$ & 22.19 & & $\mathrm{rP}$ & 238 & (15) & 8 & & $f$ & & & & & & & $\mathrm{r}$ & f & & \\
\hline $4-3$ & 23.69 & & & 93 & (2) & 46 & & & & & & & & $\mathrm{c}$ & & & & va \\
\hline 4-4 & 25.19 & & & 289 & (2) & 0 & & & & & & & & va & & & & va \\
\hline $4-5$ & 26.69 & & $\mathrm{cP}$ & 846 & (15) & 78 & & f & $\mathrm{r}$ & & & & & c & & c & & \\
\hline $4-6$ & 28.19 & & $\mathrm{cP}$ & 152 & (5) & 130 & & a & & & & & & & & & & a \\
\hline $5-1$ & 30.29 & & $\mathrm{cP}, \mathrm{rD}$ & 2286 & (10) & 986 & & a & & & & & & a & & c & & a \\
\hline $5-2$ & 31.79 & & $\mathrm{aP}, \mathrm{rD}$ & 133 & (9) & 133 & & & & & & & & c & & c & & \\
\hline $5-3$ & 33.29 & M & $\mathrm{cP}, \mathrm{rD}$ & 2011 & (15) & 203 & & c & & & & a & & c & $\mathrm{r}$ & c & & a \\
\hline $5-4$ & 34.79 & & & 1438 & (11) & 20 & & & & & $\mathrm{r}$ & & & & & & & a \\
\hline $5-5$ & 36.29 & & & 193 & (4) & 105 & & & & & & & & a & & c & & a \\
\hline $5-6$ & 37.79 & & $\mathrm{rA}$ & 722 & (10) & 77 & & $\mathrm{r}$ & & & & & & & & & & \\
\hline $6-2$ & 41.39 & & & 52 & (1) & 0 & & a & & & & & & c & & a & & a \\
\hline $6-3$ & 42.89 & & $\mathrm{rP} / \mathrm{a}$ & 1476 & (11) & 132 & & & & & & & & $\mathrm{r}$ & & c & & \\
\hline $6-4$ & 44.39 & & & 269 & (18) & 128 & & & & & & & & a & & c & & va \\
\hline $6-5$ & 45.89 & & & 10 & (1) & 10 & & $\mathrm{r}$ & & & & & & a & & & & a \\
\hline $6-6$ & 47.39 & & $\mathrm{cP}$ & 1300 & (4) & 1300 & & $a$ & & f & & f & & a & & $f$ & $\mathrm{f}$ & va \\
\hline $7-1$ & 49.49 & & & 141 & (17) & 11 & f & $\mathrm{r}$ & & & & & $a$ & c & f & & & a \\
\hline 7.2 & 50.99 & & & 153 & (5) & 0 & & & & & & f & & & & & & \\
\hline $7-3$ & 52.49 & & & 542 & (4) & 325 & & & & f & & & va & va & & & & \\
\hline $7-4$ & 53.99 & & & 216 & (1) & 0 & & & & & & & & $\mathrm{a}$ & & & $\mathrm{r}$ & $\mathrm{Vg}$ \\
\hline 7.5 & 55.49 & & & 48 & (3) & 0 & & & & & & $\mathrm{r}$ & & c & $\mathrm{r}$ & & & \\
\hline $7-6$ & 56.99 & & & 236 & (2) & 118 & & & & & & & va & & $\mathrm{r}$ & & $\mathrm{vc}$ & \\
\hline $8-1$ & 59.09 & & $\mathrm{fP}, \mathrm{rA}$ & 218 & (13) & 75 & & & & & & & & c & $\mathrm{r}$ & c & & \\
\hline $8-2$ & 60.59 & & & 0 & 0 & 279 & & & & & & & & c & & & a & a \\
\hline $8-3$ & 62.09 & & $\mathrm{rP} / \mathrm{D}$ & 54 & (1) & 108 & & & & & & & & & $\mathrm{r}$ & c & va & va \\
\hline $8-4$ & 63.59 & & $\mathrm{cP}$ & 0 & 0 & 0 & & & & & & & & va & & & va & va \\
\hline $8-5$ & 65.09 & & & 26 & (2) & 0 & & & & & & a & & & & & & a \\
\hline $8-6$ & 66.59 & & $\mathrm{fp} / \mathrm{A}$ & 772 & (8) & 14 & & $\mathrm{r}$ & & & & & & $r$ & & a & & $\mathrm{a}$ \\
\hline $9-1$ & 68.69 & & & 40 & (2) & 0 & & & & & & & & a & & & a & a \\
\hline $9-2$ & 70.19 & & $\mathrm{rP}$ & 77 & (7) & 33 & & a & & & & & & a & $\mathrm{r}$ & f & & va \\
\hline $9-3$ & 71.69 & & fP & 97 & (13) & 23 & & & & & & & va & $\mathrm{c}$ & $\mathrm{r}$ & & & va \\
\hline $9-4$ & 73.19 & & $\mathrm{rP}$ & 450 & (10) & 82 & & c & & $\mathrm{r}$ & & & & a & & & a & va \\
\hline $9-5$ & 74.69 & & & 9 & (1) & 0 & & & & c & & & & a & & & a & a \\
\hline $10-1$ & 78.29 & & & 534 & (5) & 41 & & a & & & & a & & & & & va & a \\
\hline $10-2$ & 79.79 & & $\mathrm{rP}$ & 1536 & (14) & 30 & & & & & & & & & & & & a \\
\hline $10-3$ & 81.29 & & $\mathrm{rP} / \mathrm{D}$ & 390 & (19) & 67 & & c & & f & & & & a & $\mathrm{r}$ & & & a \\
\hline $10-4$ & 82.79 & & & 146 & (4) & 104 & & c & & & & & & & & & a & a \\
\hline $10-5$ & 84.29 & & & 147 & (10) & 79 & $\mathrm{r}$ & c & & $\mathrm{r}$ & $\mathrm{f}$ & & a & a & $\mathrm{r}$ & & $a$ & \\
\hline $10-6$ & 85.79 & & $\mathrm{rP}$ & 27 & (1) & 190 & $\mathrm{r}$ & & & & & & & a & & $\mathrm{a}$ & & va \\
\hline $11-1$ & 87.89 & & & 57 & (3) & 20 & & a & & & & & & a & $r$ & $r$ & a & va \\
\hline $11-2$ & 89.39 & & $\mathrm{rP}$ & 18 & (2) & 26 & & & & & & & & a & $\mathrm{r}$ & & a & va \\
\hline $11-3$ & 90.89 & & rP & 99 & (10) & 17 & & & & & & & a & & $\mathrm{r}$ & $\mathrm{r}$ & c & a \\
\hline $11-4$ & 92.39 & & $\mathrm{rP} / \mathrm{D}$ & 51 & (5) & 132 & & $a$ & & & $\mathrm{r}$ & & & a & & c & a & a \\
\hline $12-2$ & 98.99 & & cP & 0 & 0 & 68 & & & & & & & va & & & $r$ & a & va \\
\hline $12-3$ & 100.49 & & $\mathrm{rD}$ & 867 & (7) & 102 & & & & f & & & & & & f & a & va \\
\hline $12-4$ & 101.99 & & $\mathrm{rP}$ & 251 & (7) & 0 & & c & & $\mathrm{r}$ & & & & & $\mathrm{r}$ & a & a & a \\
\hline $12-5$ & 103.49 & & cP & 969 & (7) & 31 & & f & & a & f & & & a & c & & & a \\
\hline 13-1 & 107.09 & & $\mathrm{cP}$ & 0 & 0 & 236 & & f & & & $\mathrm{r}$ & & & va & & $\mathrm{f}$ & & va \\
\hline 13-2 & 108.59 & & & 118 & (3) & 79 & & $\mathrm{r}$ & & & & & c & va & c & c & a & va \\
\hline $13-3$ & 110.09 & & $\mathrm{rP}$ & 323 & (7) & 44 & & a & & & & & & va & $\mathrm{r}$ & $\mathrm{r}$ & va & va \\
\hline $13-4$ & 111.59 & & cP & 2663 & (8) & 129 & & & & c & & & a & c & $\mathrm{r}$ & c & & $\mathrm{Vg}$ \\
\hline $14-1$ & 116.69 & & $\mathrm{rP}$ & 0 & (6) & 0 & & & & & & & va & a & & c & a & \\
\hline $14-2$ & 118.19 & & & 108 & (9) & 22 & & & & & & & a & & & & & \\
\hline $14-3$ & 119.69 & & & 693 & (2) & 0 & & & & & & & & a & & c & & a \\
\hline $14-4$ & 121.19 & & & 473 & (8) & 79 & a & & & & & & & c & $\mathrm{r}$ & & a & a \\
\hline $14-5$ & 122.69 & & & 1144 & (6) & 364 & & c & & $f$ & & & a & a & & & & \\
\hline $14-6$ & 124.19 & & $\mathrm{cP}$ & 1300 & (5) & 1647 & & & & & & & & a & & c & a & a \\
\hline Hole $611 \mathrm{C}$ & & & & & & & & & & & & & & & & & & \\
\hline $15-2$ & 119.99 & & & 468 & (8) & 337 & & a & & & & & $\mathrm{a}$ & & $\mathrm{r}$ & & a & \\
\hline $15-3$ & 121.49 & & $\mathrm{rD}$ & 475 & (7) & 429 & & & & c & & & va & & $\mathrm{r}$ & & & \\
\hline $15-4$ & 122.99 & & & 707 & (15) & 208 & & & & f & f & & & a & & & & a \\
\hline
\end{tabular}




\section{P. J. MUDIE}

Table 2 (continued).

\begin{tabular}{|c|c|c|c|c|c|c|c|c|c|c|c|c|c|c|c|c|c|c|}
\hline & Sub-bottom & & & & & & & Sp & & & & & & & & ood & & \\
\hline Section & depth (m) & $\operatorname{Pr}$ & $\mathrm{Dr}$ & Dino & (S) & Pol & $\mathrm{Pt}$ & $\mathrm{b}$ & $\mathrm{t}$ & Diat & For & Amor & $\mathrm{Cl}$ & Coal & $\mathrm{F}$ & $\mathrm{Bn}$ & Py & M \\
\hline
\end{tabular}

Hole 611C (Cont.)

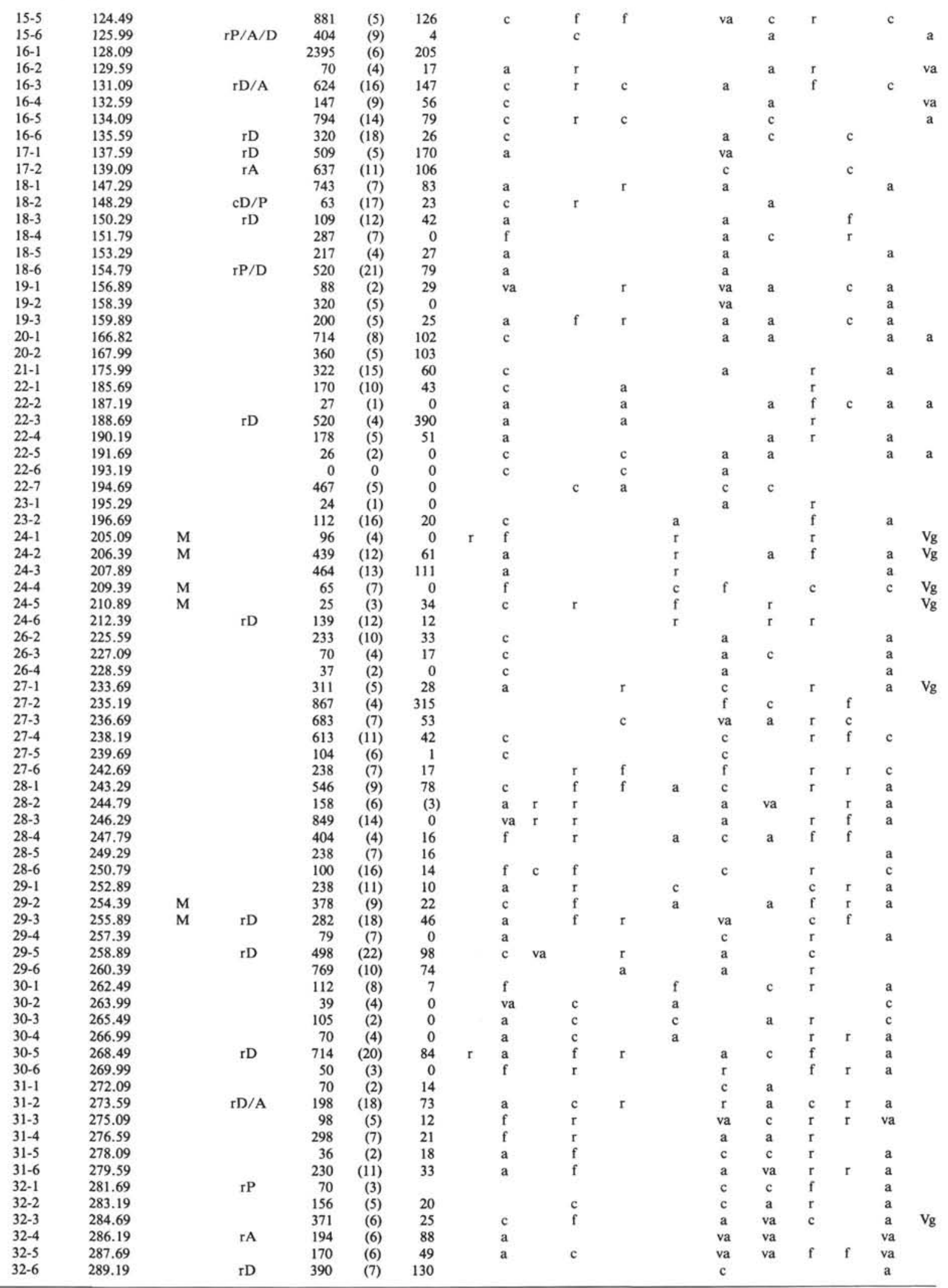


Table 2 (continued).

\begin{tabular}{|c|c|c|c|c|c|c|c|c|c|c|c|c|c|c|c|c|c|c|}
\hline \multirow[b]{2}{*}{ Section } & \multirow{2}{*}{$\begin{array}{c}\text { Sub-bottom } \\
\text { depth (m) }\end{array}$} & \multirow[b]{2}{*}{$\mathrm{Pr}$} & & & & & & $\mathrm{Sp}$ & & & & & & & & od & & \\
\hline & & & $\mathrm{Dr}$ & Dino & (S) & Pol & $\mathrm{Pt}$ & $\mathrm{b}$ & $\bar{t}$ & Diat & For & Amor & $\mathrm{Cl}$ & Coal & $\bar{F}$ & $\overline{B n}$ & Py & M \\
\hline Hole $611 \mathrm{C}($ & ont.) & & & & & & & & & & & & & & & & & \\
\hline $33-1$ & 291.29 & & & 212 & (20) & 36 & & a & & $\mathrm{r}$ & & & & a & & & a & \\
\hline $33-2$ & 292.79 & & & 127 & (8) & 22 & & $\mathrm{r}$ & & & & & a & & & $\mathrm{r}$ & & \\
\hline $33-3$ & 294.29 & & & 63 & (4) & 0 & & c & & $\mathrm{r}$ & & & $\mathrm{c}$ & c & c & $\mathrm{r}$ & a & \\
\hline $33-4$ & 295.79 & & & 71 & (3) & 0 & & c & & $\mathrm{r}$ & & & f & c & $\mathrm{r}$ & $\mathrm{r}$ & c & \\
\hline $33-5$ & 297.29 & & & 67 & (5) & 0 & & c & f & $\mathrm{f}$ & & & $f$ & & r & & c & \\
\hline $33-6$ & 298.79 & & & 104 & (5) & 30 & & c & & $\mathrm{r}$ & & & c & c & f & $\mathrm{r}$ & c & \\
\hline $35-1$ & 310.60 & & & 231 & (8) & 0 & & & & c & & & $\mathrm{f}$ & & $\mathrm{r}$ & $\mathrm{r}$ & c & \\
\hline $35-2$ & 311.99 & & & 24 & (2) & 0 & & f & $r$ & r & & & c & c & c & & c & \\
\hline $35-3$ & 313.49 & & $\mathrm{fD} / \mathrm{rA}$ & 722 & (18) & 77 & & a & & a & $\mathrm{r}$ & a & a & $\mathrm{r}$ & c & $r$ & & \\
\hline $36-1$ & 320.29 & & & 206 & (11) & 29 & & & c & r & & $\mathrm{c}$ & & & & & & \\
\hline $36-2$ & 321.79 & & & 274 & (10) & 106 & & c & c & $\mathrm{r}$ & & & c & c & c & & c & \\
\hline $36-3$ & 323.29 & & & 21 & (1) & 21 & $\mathrm{r}$ & & c & c & & c & & a & $\mathrm{r}$ & & c & \\
\hline $36-4$ & 324.79 & & rD & 130 & (15) & 9 & $\mathrm{r}$ & c & c & f & & a & & & $r$ & & a & \\
\hline $36-5$ & 326.29 & & & 86 & (4) & 86 & & & c & r & $f$ & & c & a & $\mathrm{r}$ & & a & \\
\hline $36-6$ & 327.79 & & & 230 & (8) & 16 & & f & c & c & & & c & va & c & f & c & \\
\hline $37-1$ & 329.69 & & $\mathrm{rD}$ & 214 & (12) & 71 & & & c & & & & & & f & & a & \\
\hline $37-2$ & 331.19 & & & 42 & (2) & 0 & & f & c & & & c & & c & c & & & \\
\hline $37-3$ & 332.69 & & $\mathrm{rD}$ & 143 & (4) & 54 & & a & & c & & & a & a & f & & & \\
\hline $37-4$ & 334.19 & & & 41 & (6) & 0 & & & c & c & & & f & a & & & & \\
\hline $38-1$ & 339.29 & & & 229 & (14) & 10 & & & a & c & & & & c & a & & c & \\
\hline $38-2$ & 340.79 & & & 97 & (10) & 0 & & & a & c & & & c & a & r & & & \\
\hline $38-3$ & 342.29 & & & 50 & (7) & 0 & $r$ & & c & f & & & a & a & c & & a & \\
\hline $38-4$ & 343.79 & & & 37 & (6) & 0 & & $\mathrm{r}$ & a & $\mathrm{r}$ & & a & a & a & f & & & $\mathrm{Vg}$ \\
\hline $38-5$ & 345.29 & & & 115 & (11) & 0 & & a & c & r & & & a & & a & & a & \\
\hline $38-6$ & 346.79 & & & 808 & (10) & 231 & & a & & c & $\mathrm{r}$ & & a & a & r & c & & \\
\hline $39-1$ & 348.89 & & rD & 359 & (19) & 0 & & & a & & & & a & & & & a & \\
\hline $39-2$ & 350.41 & & & 287 & (11) & 30 & & c & & f & & & a & a & $\mathrm{r}$ & c & & \\
\hline $39-3$ & 351.83 & & & 148 & (3) & 118 & & $\mathrm{f}$ & & c & & & a & a & $\mathrm{r}$ & c & & \\
\hline $39-4$ & 353.33 & & & 321 & (6) & 0 & $r$ & c & & & & & a & & f & $\mathrm{r}$ & & \\
\hline $39-6$ & 356.49 & & $\mathrm{rD}$ & 196 & (17) & 11 & $\mathrm{r}$ & a & & $\mathrm{f}$ & & & & & & & & \\
\hline $40-2$ & 359.93 & & & 333 & (9) & 30 & & $r$ & & & & & a & c & c & & a & \\
\hline $41-1$ & 367.88 & & & 111 & (5) & 0 & & c & & c & & & c & c & c & & c & \\
\hline $41-2$ & 369.59 & & & 271 & (9) & 15 & & a & & & & & a & a & c & f & & \\
\hline $41-3$ & 370.85 & MP & $\mathrm{rP}$ & 221 & (5) & 83 & & c & & & & & a & c & $\mathrm{r}$ & & c & \\
\hline $41-4$ & 372.57 & & & 310 & (9) & 0 & & a & & $r$ & & & a & a & $\mathrm{r}$ & & & \\
\hline $41-5$ & 374.53 & & & 160 & (11) & 8 & & a & & & & c & & & & & & \\
\hline $41-6$ & 375.47 & & & 106 & (4) & 15 & & c & & c & & & va & va & $r$ & & & \\
\hline $42-1$ & 377.69 & & & 203 & (3) & 0 & & c & & & & f & & c & $\mathrm{r}$ & & c & \\
\hline $42-2$ & 379.19 & & & 178 & (7) & 0 & & c & $\mathrm{r}$ & a & & c & & & $\mathrm{r}$ & & & \\
\hline $42-3$ & 380.71 & P & & 279 & (9) & 0 & & a & $\mathrm{r}$ & a & & & a & a & & & & \\
\hline $42-4$ & 382.21 & & & 308 & (8) & 68 & & f & & & & c & & c & $r$ & & & \\
\hline $42-5$ & 383.71 & & & 35 & (3) & 0 & & c & & f & & $\mathrm{f}$ & & c & $\mathrm{f}$ & & & \\
\hline $42-6$ & 385.21 & & & 141 & (8) & 0 & & a & & c & & & & & & a & a & \\
\hline $43-2$ & 388.81 & & & 155 & (8) & 28 & $r$ & & & & & & a & c & & & & \\
\hline $43-3$ & 390.47 & & & 25 & (3) & 6 & & f & & f & & & a & a & c & & & \\
\hline $43-4$ & 391.59 & & & 115 & (8) & 0 & $r$ & $\mathrm{r}$ & & f & & & & a & c & & & \\
\hline $43-6$ & 394.74 & & & 112 & (9) & 32 & & c & & $\mathrm{r}$ & & & a & c & r & & a & \\
\hline $44-1$ & 435.42 & & $\mathrm{rD}$ & 93 & (20) & 7 & & a & c & & & & & a & $\mathrm{f}$ & & a & \\
\hline $44-2$ & 436.63 & & & 77 & (7) & 22 & & $\mathrm{f}$ & r & $r$ & & & a & a & c & f & & \\
\hline $44-3$ & 438.30 & & $\mathrm{rP}$ & 708 & (15) & 58 & & f & & & & & va & & r & & & \\
\hline $44-4$ & 439.72 & & & 154 & (8) & 0 & & f & & c & & & $\mathrm{a}$ & a & c & & & \\
\hline $44-5$ & 441.28 & & $\mathrm{cD}$ & 774 & (24) & 109 & $r$ & & & & & & & & c & & & $\mathrm{Vg}$ \\
\hline $44-6$ & 442.80 & & & 666 & (18) & 79 & & f & & & & & c & c & $r$ & & & \\
\hline $45-1$ & 445.20 & & $\mathrm{rD}$ & 600 & (23) & 188 & & a & & & & & a & & & a & a & \\
\hline $45-2$ & 446.66 & & & 561 & (11) & 30 & & c & & c & & & a & a & $r$ & & & \\
\hline $45-3$ & 447.72 & & & 295 & (7) & 0 & & & c & $\mathrm{r}$ & & & $\mathrm{a}$ & $\mathrm{a}$ & $\mathrm{r}$ & & a & \\
\hline $45-4$ & 449.50 & & & 378 & (12) & 66 & & c & & & & & va & va & c & & & \\
\hline $45-5$ & 451.21 & & & 339 & (7) & 85 & & a & & c & & & $\mathrm{a}$ & va & f & & & \\
\hline $45-6$ & 452.53 & & $\mathrm{cD}$ & 1394 & (20) & 236 & & c & & f & & & a & $\mathrm{a}$ & & & a & \\
\hline $46-1$ & 495.29 & & & 493 & (6) & 0 & & r & & $\mathrm{r}$ & & & & va & $r$ & c & va & \\
\hline $46-2$ & 496.39 & & $\mathrm{rD}$ & 1206 & (16) & 264 & & a & & & & & a & & & & a & \\
\hline $46-3$ & 497.89 & & & 352 & (5) & 96 & & va & & & & & va & a & & & a & \\
\hline $46-4$ & 499.39 & & $\mathrm{rD}$ & 138 & (14) & 13 & & c & & & & & a & c & c & & & \\
\hline $46-6$ & 502.39 & & $\mathrm{rD}$ & 209 & (10) & 110 & & c & & & & & a & c & c & & & \\
\hline $47-1$ & 502.69 & & & 643 & (11) & 134 & & c & & c & $r$ & c & & $\mathrm{a}$ & c & c & c & \\
\hline $47-2$ & 504.19 & & & 2773 & (9) & 500 & & $\mathrm{f}$ & & & $\mathrm{r}$ & & a & va & & & a & \\
\hline $47-3$ & 505.69 & & $\mathrm{rD}$ & 299 & (9) & 0 & & a & & $\mathrm{r}$ & a & & a & $\mathrm{a}$ & c & $r$ & a & \\
\hline $47-4$ & 507.19 & P & $\mathrm{rD}$ & 1777 & (12) & 130 & & a & & & & & a & c & r & & a & \\
\hline $47-5$ & 508.69 & & & 494 & (19) & 39 & & a & & & & & & a & & & a & \\
\hline $47-6$ & 510.09 & & $\mathrm{rP}$ & 809 & (18) & 224 & & a & c & & & & & & c & & & \\
\hline
\end{tabular}

Note: $\mathrm{Pr}=$ preservation state $(\mathrm{M}=$ moderate, $\mathrm{P}=$ poor, unspecified $=$ good $) . \mathrm{Dr}=$ relative abundance of reworked palynomorphs $(\mathrm{P}=$ pollen and spores; $\mathrm{D}=$ dinocysts; $\mathrm{A}=$ acritarchs); Dino = number of dinocysts $/ \mathrm{g}$. Relative abundances: phytoliths $(\mathrm{Pt})$; algal spores $(\mathrm{Spor})$ of the spherical black type (b) and the translucent type (t); pyritized diatom fragments (Diat); foraminifers (For); amorphogen (Amor); clay flocs (Cl); carbonized organic fragments $(\mathrm{Coal})$; wood particles $(\mathrm{F}=$ nonlignified fibers; $\mathrm{Bn}=$ brown lignified fragments); pyrite (Py); and mineral particles $(\mathrm{M})$, including volcanic glass $(\mathrm{Vg})$. Relative abundance scale: $\mathrm{r}=\operatorname{rare}(<5 \%) ; \mathrm{f}=$ frequent $(5-15 \%) ; \mathrm{c}=\operatorname{common}(>15-30 \%) ; \mathrm{a}=$ abundant $(>30-50 \%) ; \mathrm{va}=$ very abundant $(>50 \%)$. 

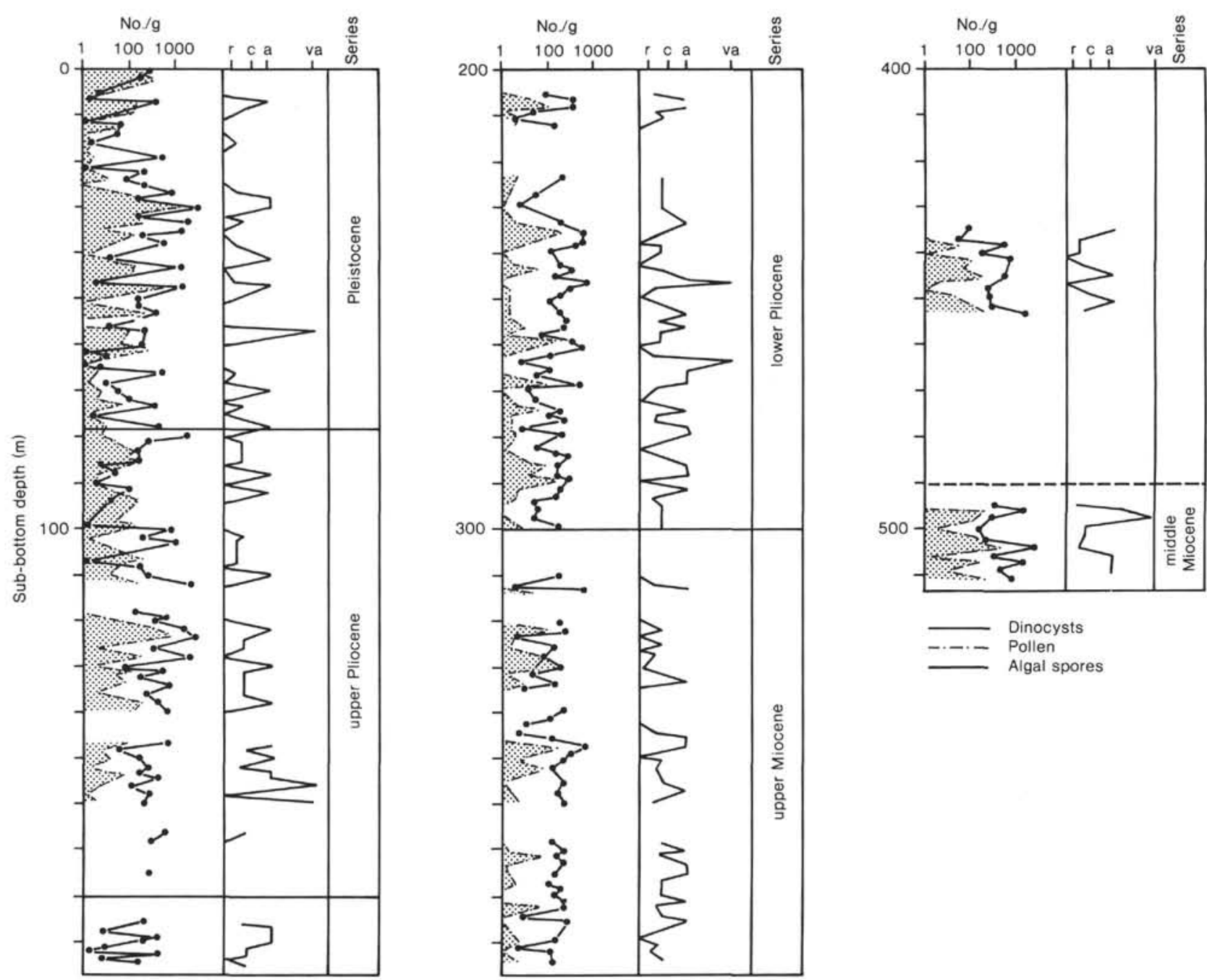

Figure 5. Dinocyst and pollen concentrations (number per gram) and relative abundances of algal spores plotted against depth and series in Holes 611 and $611 \mathrm{C}$. Note that the palynomorph abundance scale is logarithmic; relative abundances are $r=\operatorname{rare}(<5 \%) ; f=$ frequent $(5-15 \%) ; \mathrm{c}=$ common $(>15-30 \%) ; \mathrm{a}=$ abundant $(>30-50 \%) ; \mathrm{va}=$ very abundant $(>50 \%)$.

tent pattern of dinoflagellate frequency oscillations in the upper Pliocene interval for which overlapping samples from the drift crest (Hole 611, Sections 14-3 to 146) and trough (Hole 611C, Sections 15-1 to 15-5) were available. In contrast, the pattern shown by pollen and spores is much more erratic, and more investigation is needed to determine whether there is a tendency for pollen to be winnowed from the sediment wave crests and redeposited in the troughs.

Seventy-two of the most productive samples from Holes 611 and $611 \mathrm{C}$ were used to construct a tentative dinoflagellate biostratigraphy for Site 611. Figure 6 shows the distributions of the 67 recognized taxa and their ranges relative to the chronostratigraphy reported for Site 611 (Site 611 report, this volume, fig. 13). Three informal dinocyst concurrent-range zones and two subzones were delimited for Site 611 , using the criteria described earlier for Site 607. The major components of the concurrent-range zones at Site 611 and 607 are the same, but there are some conspicuous differences in details, which are described in the following discussion.
Dinocyst Zone I in Hole $611 \mathrm{C}$ is distinguished primarily by the presence of an association of species similar to that which characterizes Zone I of Site 607; these species include Achomosphaera andalousiense, Tectatodinium simplex, Invertocysta spp., ?Operculodinium echigoense, Lingulodinium machaerophorum, and, in the upper part of Zone I, Operculodinium crassum. The boundary between Zones I and II in Hole $611 \mathrm{C}$ is marked by the LADs of Nematosphaeropsis aquaeducta and Nematosphaeropsis sp. 1, in Sample 611C-26-2, 48-50 cm. The LADs of $A$. andalousiense, Labyrinthodinium truncatum, and ?Cannosphaeropsis $\mathrm{sp} .1$ occurs just below the Zone I/II boundary in Section $611 \mathrm{C}-27-4$ or $611 \mathrm{C}$ 27-5.

Dinocyst Zone I in Hole $611 \mathrm{C}$ can be subdivided only tentatively because of poor recovery of sediments between $495 \mathrm{~m}$ (Section 611C-46-1) and $452 \mathrm{~m}$ (Section 611C-45-6). The boundary between Subzones Ia and Ib in Hole $611 \mathrm{C}$ is placed in Sample 611C-45-6, 62-64 cm, because this level marks the FADs of several taxa-Amiculosphaera umbracula, Pyxidiella sp. 1, and Nemato- 


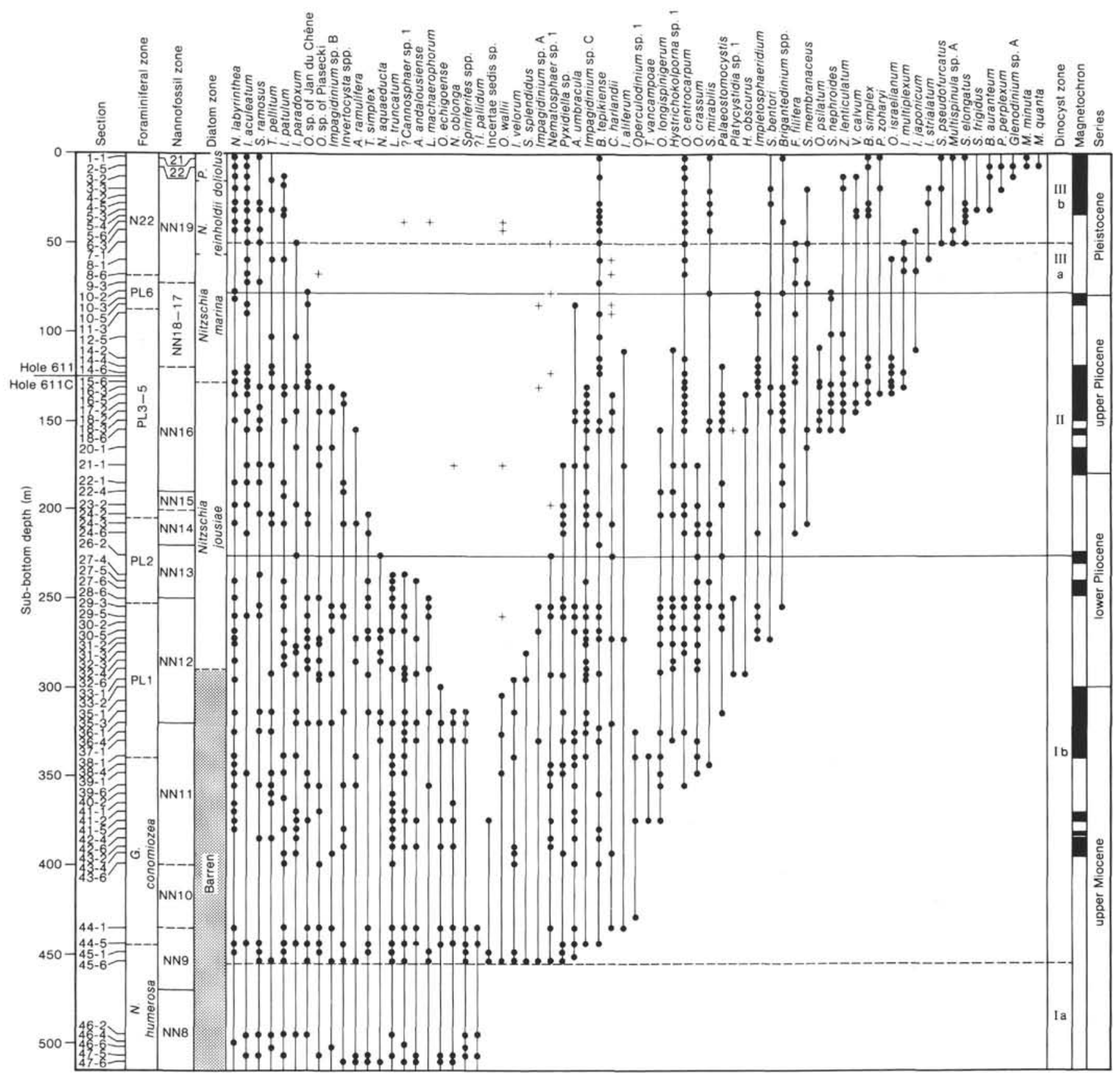

Figure 6. Range and distribution chart of dinoflagellates recovered from Neogene to Pleistocene sediments in Holes 611 and $611 \mathrm{C}$. Dots indicate presence of well-preserved specimens; + indicates rare, poorly preserved specimens, probably reworked. Foraminiferal, calcareous nannofossil, and diatom zones, and magnetostratigraphy and age determinations, are from Leg 94 site reports (this volume). Magnetochrons: shaded = normal polarity; unshaded $=$ reversed polarity.

sphaeropsis sp. 1- that first appear near the base of the planktonic foraminiferal Globoratalia conomiozea Zone in Hole $611 \mathrm{C}$, and because these dinoflagellates mark the boundary between Subzones Ia and Ib at a similar datum in Hole 607A. At Site 611, the following taxa also have their FADs at or just above the Subzone $\mathrm{Ia} / \mathrm{Ib}$ boundary: Incertae sedis sp. I of Edwards (1984), Operculodinium wallii, Impagidinium velorum and I. aliferum, Spiniferites splendidus, Impagidinium sp. A., Impagidinium sp. C, Bitectatodinium tepikiense, and Corrudinium harlandii. Three taxa have restricted ranges in
Subzone Ib of Hole 611C: Incertae sedis sp. I of Edwards (1984), Tuberculodinium vancampoae, and Platycystidia(?) sp. 1 of Manum (1976). As in Subzones Ib of Site 607, Operculodinium wallii, ?O. echigoense, and $L$. machaeropherum also have their LADs near the Miocene/Pliocene boundary in Hole 611C. But at Site 611, unlike Site 607, Nematosphaeropsis oblonga, and Impagidinium velorum also disappear close to the Miocene/ Pliocene boundary in Subzone Ib of Hole 611C.

Numerous small, taxonomically obscure cysts have their FADs in the middle of Subzone Ib in Hole 611C, and 
they subsequently become important constituents of the Zone II (upper Pliocene) assemblages at Site 611; these cyst forms are referred to here as Hystrichokolpoma sp. 1 and Impletospheridium spp. Other taxa which have their FADs near the top of Subzone Ib in Hole 611C include Palaeostomocystis spp. of Baltes (1971), Hystrichosphaeropsis obscurum, S. bentori, and Brigantedinium spp.

The base of Hole 611C (and nominal base of dinocyst Subzone Ia) has an age of about $11 \mathrm{Ma}$, and the age of the boundary between Subzones Ia and Ib at Site 611 is probably about $9.5 \mathrm{Ma}$ on the basis of its occurrence in the middle of coccolith Zone NN9. As at Site 607, Subzone Ib of Hole 611C spans the Miocene/Pliocene boundary and terminates at the top of Zone NN13, which is dated in Hole $611 \mathrm{C}$ by the top of the Nunivak Subchronozone as $4.10 \mathrm{Ma}$. The age of the Zone I/II boundary at Site 611 is therefore about $0.5 \mathrm{~m}$.y. younger than at Site 607.

Dinocyst Zone II at Site 611 is distinguished primarily by the presence of Amiculosphaera umbracula, Corrudinium harlandii, Impagidinium aliferum, and Operculodinium sp. of Jan du Chêne (1977), all of which have their LADs at or near the Zone II/III boundary, placed in Sample 611-10-3, 48-50 cm. Other frequently occurring taxa are Filisphaera filifera and Spiniferites membranaceus, both of which have their FADs just above the Zone I/II boundary. Common taxa in the lower half of Zone II also include Achomosphaera ramulifera, Invertocysta spp. (mainly $I$. lacrymosa), Operculodinium sp. of Piasecki (1980), O. longispinigerum, Impagidinium sp. B, Impagidinium sp. C, and Palaeostomocystis spp. of Baltes (1971), all of which have their LADs near the middle of Zone II (in Samples 611C-18-6, 48-50 cm, to $611 \mathrm{C}-15-2,48-50 \mathrm{~cm})$. As in Zone II at Site 607, Operculodinium crassum, and $O$. longispinigerum have their LADs in Zone II near the boundary between the lower and upper Pliocene (Sample 611C-21-1, 48-50 cm). Other taxa that disappear near this boundary in Hole $611 \mathrm{C}$ are Hystrichokolpoma sp. 1 and Pyxidiella sp. 1.

Several peridinioid taxa make their first appearances in the upper half of Zone II, beginning in Sample 611C$17-2,48-50 \mathrm{~cm}$, corresponding to the Kaena Subchron, the base of which has an age of $2.99 \mathrm{Ma}$. These taxa include Brigantedinium simplex, Zygabikodinium lenticulatum, Votadinium calvum, and Selenopemphix nephroides. The LAD of $S$. nephroides occurs at the Zone II/III boundary at Site 611. The first appearance of Polysphaeridium zoharyi (Sample 611C-16-3, 48-50 cm) occurs near the top of Zone II, as at Site 607. At Site 611 , however, the FAD of $P$. zoharyi is accompanied by the FADs of Operculodinium israelianum and Impagidinium multiplexum; the latter two species either appear at the Pliocene/Pleistocene boundary in Hole $607(O$. israelianum) or were not found at the southern site ( $I$. multiplexum).

Dinocyst Zone II at Site 611 extends from the top of the Nunivak Subchron (4.10 Ma) to the top of the Olduvai Subchron $(1.66 \mathrm{Ma})$, which lies at the Pliocene/ Pleistocene boundary; hence, the top of the palynozone apparently has a slightly younger age than at Site 607 , where the dinocyst Zone II/III boundary lies below the Olduvai Subchron and has an age of between 1.90 and 2.20 Ma.

Dinocyst Zone III at Site 611 has a relatively low diversity of species, dominated by Nematosphaeropsis labyrinthea, Impagidinium aculeatum, Operculodinium centrocarpum, Bitectatodinium tepikiense, Spiniferites mirabilis, Brigantedinium spp., and $B$. simplex. Zone III is tentatively subdivided into lower and upper Pleistocene subzones on the basis of the LADs of several diagnostic Neogene taxa in Sample 611-7-1, 48-50 cm and the FADs of characteristic subarctic-arctic Pleistocene to Recent taxa (Harland, 1983; Mudie and Short, 1985), for example, Spiniferites elongatus and Multispinula sp. A. The boundary between Subzones IIIa and IIIb is marked by the LADs of Impagidinium paradoxum, I. multiplexum, and Filisphaera filifera in Sample 611-7-1, $48-50 \mathrm{~cm}$. At Site 611, the Subzone IIIa/IIIb boundary lies just above the base of nannofossil Zone NN19, and it has an extrapolated age of about 1.5 Ma. Operculodinium israelianum has its last occurrence in Subzone IIIa at Site 611.

The base of Subzone IIIb at Site 611 is marked by the FADs of Spiniferites cf. pseudofurcatus, Multispinula sp. A, and Spiniferites elongatus. The FAD of Impagidinium strialatum occurs just below the Subzone IIIa/b boundary, and the LAD of this species occurs near the middle of Subzone IIIb (in Section 611-8-1). The following species also have their LADs near the middle of Subzone IIIb: Tectatodinium pellitum and Impagidinium patulum (Section 611-3-1), Spiniferites membranaceus (Section 611-3-3), and Zygabikodinium lenticulatum (Section 611-3-1). The LAD of Votadinium calvum occurs in Section 611-5-3, which also marks the FADs of Spiniferites frigidus and Brigantedinium auranteum. Four taxa have restricted distributions at the top of Subzone IIIb: Piperodinium perplexum, Glenodinium sp. A, Multispinula minuta, and M. quanta.

\section{COMPARISONS}

There is little published work on the Neogene and Pleistocene cysts from deep-sea sediments with which to compare the biostratigraphic data from Sites 607 and 611. Figure 1, however, shows the locations of European stratotypes, parastratotypes, and DSDP sites in the North Atlantic for which dinoflagellate stratigraphies have been described. Comparisons can also be made with Neogene stratotypes in central Honshu, Japan, and with Neogene to Pleistocene DSDP holes in the Bering Sea.

Habib (1972) described the most common dinoflagellates in upper Cenozoic sediments from Holes 104, 105, and 106 of DSDP Leg 11. Most of these dinoflagellates were long-ranging (Eocene-Pleistocene) species, found at Sites 607 and 611, including Achomosphaera ramulifera, Operculodinium centrocarpum, Leptodinium ( = Impagidinium) aculeatum, L. patulum, L. paradoxum, Nematosphaeropsis labyrinthea, Spiniferites ramosus, and Tectatodinium pellitum. Habib (1972) noted, however, that Hystrichosphaeropsis obscurum appeared to have its LAD in upper Miocene sediments. The sporadic occurrence of this taxon in lower Pliocene sediments of 
Site 611 may therefore indicate reworking. Habib (1972) also noted that Achomosphaera ramulifera appeared to be a useful guide species because it did not occur in sediments younger than Pliocene/lower Pleistocene. This observation is further supported by the ranges of $A$. ramulifera at Sites 607 and 611.

Piasecki (1980) described the dinoflagellates from the Hodde and Gram formations in Jylland, western Denmark. Planktonic foraminifers near the top of the Gram Formation in the Gram borehole apparently correspond to Zones N15 to N16 (upper Miocene); these data and molluscan faunas were used to assign middle and late Miocene ages to the Hodde and Gram formations, respectively. The dinoflagellate flora in the Gram borehole clearly represents a littoral environment, and typical deep-sea taxa such as Nematosphaeropsis labyrinthea and Impagidinium spp. are not present; therefore, only limited comparison with the Leg 94 sites is possible. Piasecki (1980) also noted that comparison of the Danish dinocyst flora and the Neogene dinocyst stratigraphy (Harland, 1978) from the northwest European continental shelf and adjacent areas revealed (1) the absence of some Miocene guide species from the Hodde and Gram formations, and (2) some differences between the ranges of taxa in the continental shelf holes and the Danish reference sections.

At the Danish middle to upper Miocene type locality, however, it is notable that Nematosphaeropsis aquaeducta Piasecki and Labyrinthodinium truncatum Piasecki have their LADs at the middle/upper Miocene boundary, and that Achomosphaera andalousiense Jan de Chêne has its FAD near this boundary both in Denmark and on the northwest European shelf. Operculodinium centrocarpum, Operculodinium sp. of Piasecki (1980), Lingulodinium machaerophorum, Spiniferites pseudofurcatus, and Hystrichosphaeropsis obscurum range throughout the Miocene in the Gram borehole. Tectatodinium pellitum and T. psilatum sensu Piasecki $1980(=T$. simplex [Harland] Edwards) have their FADs below the middle/ upper Miocene boundary, and their ranges extend almost to the top of the section.

Jan du Chêne (1977) studied the dinoflagellates in the middle and upper Miocene sediments from Carmona, Arroyo Galapagar, and Vejer de la Frontera in Spain, north of the Strait of Gibraltar. The Carmona site is the stratotype for the Andalousian Stage, which was defined by Perconig (1964) as the equivalent of the Messinian marine beds. Perconig (1973), however, later expanded the definition of the Andalousian Stage to include nannofossil Zones NN10, NN11, and the base of NN12, and the Andalousian Stage was assigned an age range of 10 to $4 \mathrm{Ma}$, thereby including most of the middle to upper Miocene Tortonian Stage and the whole upper Miocene Messinian Stage. According to Berggren (1981), the uppermost Andalousian Stage would now also include part of the lower Pliocene Zanclean Stage, which has an age of 5.4 to $3.4 \mathrm{Ma}$.

The dominant dinocysts throughout the Miocene Carmona beds are Operculodinium centrocarpum, Lingulodinium machaerophorum, and Spiniferites spp. Typical deep-sea taxa are also represented by $N$. labyrinthea, sev- eral Leptodinium (= Impagidinium) species, and Thalassiphora sp. cf. T. pelagica Habib 1971 (= Invertocysta lacrymosa Edwards 1984). The holotype of Achomosphaera andalousiense is restricted to sediments of middle late Miocene age at Carmona. Hystrichosphaeropsis obscurum and $A$. ramulifera are occasionally present. $O p$ erculodinium sp. of Jan du Chêne (1977) and cf. Pyxidiella sp. of Jan du Chêne (1977) are present throughout the Tortonian and Andalousian stages at Carmona; the latter taxon is similar to Pyxidiella sp. 1 at Sites 607 and 611.

Habib (1971) described the dinoflagellates across the Miocene/Pliocene boundary in the Tabianian stratotype at Tabiano Bagnia, northern Italy. He reported that the most common species included $O$. centrocarpum s.l., Achomosphaera ramulifera s.l., and Spiniferites cf. ramosus, and that the deep-sea taxa $N$. labyrinthea, Leptodinium aculeatum, $L$. patulum, $L$. paradoxum, and Thalassiphora $\mathrm{cf}$. pelagica (= Invertocysta lacrymosa Edwards) were sporadically present. It is notable that Habib's illustrations of $O$. centrocarpum s.l. show cysts that are more similar to $O$. echigoense Matsuoka and $O$. crassum Harland than to the holotype and paratypes of O. centrocarpum (Deflandre and Cookson) Wall, 1967.

Baltes (1971) studied the dinoflagellates in the lower Pliocene deposits (Lower Pontian Stage) of Romania. These sediments were deposited in a shallow marine littoral basin overlying lacustrine/fluviatile sediments, and few of the species present have been reported from studies of deep-sea sediments. Of interest, however, is the occurrence of several spherical cysts with quadrate to hexagonal archeopyles (Baltes, 1971, pl. 4, figs. 8-10) which resemble some common Pleistocene to Recent Brigantedinium spp., and the presence of Palaeostomocystis spp. (Baltes, 1971, pl. 5, figs. 3-12), which appear to have transapical archeopyles and resemble the peridinioid genus Votadinium Reid. Some of the Brigantedinium spp. and Palaeostomocystis spp. in the upper Miocene to lower Pliocene sediments from Site 611 appear to be similar to these Pontian Stage taxa.

Dinoflagellates in lower Pleistocene sediments from the Royal Society borehole at Ludham, Norfolk, England, were studied by Wall and Dale (1968). Sediments at the base of the hole (foraminiferal Zones LI, LII and LII) were distinguished by the presence of Leptodinium (= Impagidinium) multiplexum, which has its LAD in Zone LIII at the top of the Ludhamian Stage. Pollen from the Ludham borehole indicates that the English Ludhamian Stage is equivalent to the Netherlands Tiglian Stage; according to Harland et al. (1982), the Ludhamian Stage corresponds to the Jaramillo Subchron (0.94$0.88 \mathrm{Ma}$ ) and to the lower part of the lower middle Pleistocene Emilianian Stage of southern Europe. The distribution of $I$. multiplexum in DSDP Hole 611 is in good agreement with this age.

Harland (1979) erected a tentative dinoflagellate zonation for middle Miocene to Recent sediments from DSDP Site 400 , which lies in $4399 \mathrm{~m}$ of water at the base of the continental slope in the Bay of Biscay. He compared the taxa in 27 samples used for this stratigraphy with those from Miocene to Pleistocene sediments 
on the Rockall Plateau (DSDP Sites 403-406) reported by Costa and Downie (1979); with those from middle Miocene sediments of the Vøring Plateau (DSDP Site 338), reported by Manum (1976); and with those from wells on the Nova Scotian Shelf, reported by Williams (1975).

At Site 400 , the middle/upper Miocene boundary marks the top of Harland's dinocyst Zone IIa, which is characterized by the disappearance of the middle Miocene species Pentadinium taeniagerum and Palaeocystodinium golzowense. The absence of these taxa at Site 611 supports a latest middle Miocene age for the base of Hole 611C. Amiculosphaera umbracula has its FAD at the base of Zone IIa at Site 400, but it does not appear below the middle upper Miocene at Sites 607 and 611 .

Harland's dinocyst Zone IIb extends from the middle/upper Miocene boundary to the top of Zone NN13 in Hole 400A; hence, it spans the Miocene/Pliocene boundary and corresponds to the same time interval as dinocyst Zone Ib at Sites 611 and 607. Many of the dinocyst taxa in Zone IIb at Site 400 are the same as at Sites 611 and 607; conspicuous differences are the absence of Nematosphaeropsis spp., lower diversity of Impagidinium spp., and higher diversity of Spiniferites spp. at Site 400. Nematosphaeropsis labyrinthea and Impagidinium spp. are abundant, however, in the upper Miocene sediments described by Edwards (1984) for DSDP Leg 81 Sites 552, 554, and 555 on the Rockall Plateau, thereby suggesting that the Bay of Biscay site represents late Miocene to early Pliocene oceanic conditions somewhat different from those in the region south of the Norwegian Sea (Rockall Plateau and Gardar Ridge) or on the west flank of the Mid-Atlantic Ridge near the Azores (Site 607).

Harland's dinocyst Zone III at Site 400 extends from NN13 to the middle of NN19 (middle Pleistocene), and it is therefore similar in age to dinocyst Zones II and IIIa at Sites 607 and 611. The base of Zone III at Site 400 is marked by the FADs of $N$. labyrinthea, Spiniferites belerius, and Tectatodinium pellitum. As in Zone II of Sites 607 and 611, O. crassum Harland, Spiniferites splendidus, ?Ellipsoidinium sp. (= Corrudinium harlandii Matsuoka), and Thalassiphora delicta sensu Harland (= Invertocysta lacrymosa Edwards) have their LADs in Zone III at Site 400. Amiculosphaera umbracula has its LAD at the top of Zone III at Site 400, whereas its LAD at Sites 611 and 607 is at the top of Zone II, in the upper Pliocene. Protoperidinium subinerme $(=$ Selenopemphix nephroides) also has its FAD in the upper Pliocene at Sites 400, 611, and 607.

Harland's (1979) dinocyst Zone IV (middle Pleistocene to Recent) at Site 400 contains assemblages similar to those in the upper Pleistocene Zone IIIb at Sites 607 and 611. Notable differences, however, are the absence of Trinovantedinium capitatum and Stelladinium stellatum and the persistent presence of Tectatodinium pellitum in the upper Pleistocene sediments at Sites 607 and 611. Zone IIIb at Sites 611 and 607, however, includes two middle to late Pleistocene taxa not reported by Harland (1979), viz. B. auranteum and Zygabikodinium lenticulatum, and two cysts of uncertain affinity, Piperodi- nium perplexum and Glenodinium sp. A; all of these taxa are common in Recent sediments of the western North Atlantic or Canadian Arctic Archipelago (Mudie and Short, 1985).

Matsuoka (1983) described Neogene and Pleistocene dinocysts from the Niigata Sedimentary Basin on the west coast of central Honshu, along the Sea of Japan. Matsuoka (1983) reports that foraminifers, calcareous nannoplankton, diatoms, and pollen allow the upper Cenozoic formations of the Niigata District to be correlated with corresponding formations in other parts of the world, including the North Atlantic. Matsuoka (1983) recognized two Neogene to Pleistocene dinoflagellate assemblage zones in the Niigata formations: (1) a Transitional Assemblage Zone, middle Miocene to lower Pliocene, which is correlative with Harland's (1979) Zone II at Site 400 and with Costa and Downie's (1979) middle to upper Miocene Zone VIII for DSDP Sites 403 to 406; and (2) a Younger Assemblage Zone, lower upper Pliocene to upper Pleistocene, which is correlative with Harland's (1979) Zones III and IV and with Costa and Downie's (1979) Pleistocene Zone IX.

In the Japanese sections, the Miocene to lower Pliocene Transitional-Zone cyst assemblages are dominated by $O$. echigoense, Achomosphaera ramulifera, $N$. labyrinthea, Lingulodinium machaerophorum, and Spiniferites spp., as in Zone I at Sites 611 and 607. O. longispinigerum, $O$. crassum, Spiniferites mirabilis, $O$. wallii, and Impagidinium paradoxum are also present, as at Sites 607 and 611; but I. patulum is rare and other Impagidinium spp. are absent. In the lower Pliocene to upper Pleistocene Younger Assemblage Zone, O. centrocarpum, A. ramulifera, Spiniferites membranaceus, $S$. mirabilis, and Tectatodinium pellitum are abundant, and $O$. longispinigerum, $N$. labyrinthea, S. elongatum, and other Spiniferites species are common throughout the interval. Impagidinium species, including $I$. paradoxum, I. patulum, and $I$. japonicum, become common in this zone, and $I$. aculeatum and $I$. strialatum are also present, as in Zones II to III at Sites 611 and 607. Notable, however, is the absence of protoperidinioid cysts in the upper Neogene and Pleistocene sediments of the Niigata Basin.

In contrast, Bujak (1984) reports the occurrence of several protoperidinoid genera in upper Miocene to upper Pleistocene sediments from DSDP Leg 19 Sites 183 to 192 , in the Bering Sea. Bujak (1984) proposed an Eocene to Pleistocene dinocyst-acritarch zonation for these sites which was calibrated with other microfossil zonations. Four concurrent-range zones were delimited for the upper Miocene to Pleistocene sections, as follows: (1) Spiniferites ovatus Zone (upper Miocene-lower Pliocene), which includes Filisphaera filifera, Impagidinium pallidum, I. velorum, Brigantedinium spp., Selenopemphix nephroides, and Tuberculodinium vancampoae and contains the LAD of Cannosphaeropsis; (2) Impagidinium pacificum Zone (lower Pliocene-upper Pliocene), which includes the LADs of $I$. velorum, $I$. pallidum, and $T$. vancampoae; (3) Nematosphaeropsis lemniscata Zone (upper Pliocene-lower Pleistocene), which includes the LADs of Filisphaera filifera and S. nephroides; (4) Spiniferites frigidus Zone (lower Pleistocene-upper Pleis- 
tocene), which includes Spiniferites frigidus and Multispinula minuta. It is notable that the boundaries of $\mathrm{Bu}-$ jak's four zones correspond closely to the ages of the boundaries of Zones Ia/Ib, I/II, II/III, and IIIa/IIIb at Sites 607 and 611, and that the ranges of $T$. vancampoae, $F$. filifera, Brigantedinium spp., S. nephroides, $S$. frigidus, and $M$. minuta are approximately the same in the Bering Sea holes as at the high-latitude North Atlantic Site 611.

\section{PALEOECOLOGICAL INTERPRETATION}

Paleoecological interpretation of palynological data from Sites 607 and 611 is presently limited by the following constraints:

1. The taxonomic positions and evolutionary affinities of many North Atlantic Neogene dinocyst species are still too uncertain to permit detailed comparison of assemblages reported for paralic (Piasecki, 1980), shelf (Williams, 1975; Harland, 1978), continental rise (Harland, 1979), and deep-sea environments of high latitudes (Manum, 1976; Costa and Downie, 1979) and middle latitudes (Habib, 1972).

2. Palynological preparation methods, especially sieve mesh size and use of oxidants, vary among different workers and significantly affect the recovery of small species $(<20 \mu \mathrm{m})$ and the appearance of thin-walled taxa.

3 . The periodic fluctuations in palynomorph concentrations shown in Figures 3 and 5 indicate that dinocyst and pollen concentrations are strongly affected by cyclical ecological events (climatic or depositional) in upper Miocene to Quaternary deep-sea sediments of both the subtropical and subpolar sites; hence, valid comparison of paleoecological conditions in different geographic areas of the North Atlantic can be made only when it is known that the same phases of the cyclical events are being compared.

4. Although the periodic fluctuations in dinocyst and pollen concentrations observed at Sites 607 and 611 suggest a response at periodicities within the range of the eccentricity component of Milankovitch-type orbital variations, more closely spaced samples and spectral-analysis statistics are needed to verify this correlation.

Despite these limitations, a few general paleoecological comments can be made:

1. Throughout the stratigraphic sections at both Site 607 and Site 611, the most abundant and frequently occurring dinocysts are extant gonyaulacoid species that have very widespread distributions (Harland, 1983; Mudie, 1980; Mudie and Short, 1985) in modern North Atlantic deep-sea sediments: Nematosphaeropsis labyrinthea, Impagidinium aculeatum, I. paradoxum, I. patulum, Operculodinium centrocarpum, and Bitectatodinium tepikiense. These long-ranging species appear to be good markers of pelagic conditions and tolerant of a wide range of sea surface temperatures, namely, subtropical to subarctic.

2. It is notable that North Pacific species (Matsuoka, 1983; Bujak, 1984) of Impagidinium, including I. japonicum, ?I. pallidum, and I. velorum, are present in the North Atlantic in Miocene to Pliocene sediments, up to the top of the Gauss Chronozone, corresponding to about
$2.47 \mathrm{Ma}$ ), by which time they all disappeared (Figs. 4 and 6). The apparent North Atlantic extinction of the Pacific Impagidinium species may be related to the formation of the Panama Isthmus at about 3.5 Ma (Berggren, 1981).

3. A reciprocal pattern is found in the North Atlantic distributions of two widespread Bering Sea species (Bujak, 1984), Filisphaera filifera and Selenopemphix nephroides. At Sites 607 and 611 (Figs. 4 and 6), the first common occurrence of $F$. filifera and the FAD of $S$. nephroides are in the upper Pliocene (about 3.5 Ma). The FAD of $F$. filifera in the Arctic Ocean also has an age of between 3 and 4 Ma (Mudie, 1985; Aksu and Mudie, 1985). This suggests that there may have been an increased inflow of Pacific Ocean water to the North Atlantic after the formation of the Panama Isthmus and before the closure of Bering Strait during Pleistocene glaciations.

Table 3 summarizes the range data for dinoflagellate cysts at Sites 607 and 611, and it includes suggested temperature tolerances of selected taxa based on their first occurrences and/or the timing of their last occurrences at the subtropical and subarctic locations. A few species notably associated with the Messinian salinity crisis (ending at $5.3 \mathrm{Ma}$ ) are also tentatively denoted as salinitysensitive. In view of the present lack of data on ecological tolerances of North Atlantic Neogene dinocysts (Harland, 1979; Edwards, 1984), it is believed that further speculation on the ecological, phytogeographical, and evolutionary trends of the Leg 94 data is not warranted. The data in Table 3, however, clearly show that many typical deep-sea taxa (e.g., Impagidinium and Nematosphaeropsis) disappeared about 1 to $0.5 \mathrm{~m}$.y. earlier at Site 611 than at Site 607, presumably reflecting the earlier onset of cold surface-water conditions at the high-latitude site. In addition, the first appearances of $F$. filifera and several protoperidinioid taxa which are common in Recent arctic/subarctic shelf sediments (e.g., Brigantedinium spp., Multispinula spp.) occur 2.5 to 0.5 m.y. earlier at Site 611, presumably reflecting a combination of earlier surface-water cooling and sea ice cover at the high-latitude site.

\section{SYSTEMATIC DESCRIPTIONS}

Sixty-seven dinoflagellate cyst taxa were identified; all the species are listed below, with descriptions or remarks where appropriate. Synonyms for most of the species are listed in Lentin and Williams (1981). The illustrated specimens are designed with GSC number and England Finder coordinate, and are registered in the palynological collection of the Environmental Marine Geology Subdivision of the Atlantic Geoscience Centre at Bedford Institute of Oceanography, Dartmouth, Nova Scotia.

\author{
Division PYRROPHYTA Pascher, 1914 \\ Class DINOPHYCEAE Fritsch, 1929 \\ Order PERIDINIALES Haeckel, 1894
}

\section{Genus ACHOMOSPHAERA Evitt, 1963}

Achomosphaera andalousiense Jan du Chêne, 1977 (Plate 2, Fig. 6). This species was first described on the basis of abundant specimens in the lower Carmona beds (upper Miocene) of Southern Spain by Jan du Chêne (1977, p. 122, pl. 1, figs. 1-4), who distinguished it primarily by its long fine spines with very complex process tips, most of which are branched to form a closed reticular 
Table 3. Summary of dinoflagellate range data for Sites 607 and 611 , with paleoecological annotations: $(+)$ cold-sensitive; (s) salinity-sensitive; $(-)$ moderately cold-tolerant; $\left({ }^{*}\right)$ highly cold-tolerant.

\begin{tabular}{|c|c|c|}
\hline Dinocyst zone/subzone & Site 607 & Site 611 \\
\hline Zone 1: approximate age: & $7.1-4.7 \mathrm{Ma}$ & $11-4.1 \mathrm{Ma}$ \\
\hline A. andalousiense & Top & Top \\
\hline N. aquaeducta & Top & Top \\
\hline$N$, oblonga & Top & Top \\
\hline Incertae sedis sp. 1 & Top & Top \\
\hline ?Operculodinium sp. 1 & Top & Top \\
\hline$(+)$ L. truncatum & & Top \\
\hline Subzone Ib: approximate age: & $6-4.7 \mathrm{Ma}$ & $10.5-4.1 \mathrm{Ma}$ \\
\hline Nematosphaeropsis sp. 1 & Range and top & Range and top \\
\hline Cannosphaeropsis sp. 1 & Range and top & Range and top \\
\hline Platycystidia(?) sp. 1 & Range and top & Range \\
\hline (*) Pyxidiella sp. 1 & Range and top & Base \\
\hline (s) O. wallii & Top $(5.3 \mathrm{Ma})$ & Top (5.3 Ma) \\
\hline (s) ?O. echigoense & Top (5.3 Ma) & Top $(5.3 \mathrm{Ma})$ \\
\hline (s) L. machaerophorum & Top $(5.3 \mathrm{Ma})$ & Top (5.3 Ma) \\
\hline (s) T. vancampoae & & Range \\
\hline Zone II: approximate age: & $4.7-2.0 \mathrm{Ma}$ & $4.1-1.7 \mathrm{Ma}$ \\
\hline A. umbracula & Top (2.0 Ma) & Top (1.9 Ma) \\
\hline Invertocysta spp. & Top (2.48 Ma) & Top (2.75 Ma) \\
\hline (s) Paleostomocystis(?) sp. & Top (3.4 Ma) & Top (2.5 Ma) \\
\hline O. longispinigerum & Top (3.4 Ma) & Top (2.4 Ma) \\
\hline O. crassum & Top (3.4 Ma) & Top (3.4 Ma) \\
\hline (*) Pyxidiella sp. 1 & & Top \\
\hline T. simplex & Top & Top \\
\hline I. aliferum & Range & Top \\
\hline (-) I. japonicum & Range & Base \\
\hline P. zoharyi & Base (3.4 Ma) & Base (3.4 Ma) \\
\hline$(+)$ S. nephroides & Base (2.5 Ma) & Range (3-1.7 Ma) \\
\hline$(+)$ O. psilatum & Base & Range (3-2.3 Ma) \\
\hline (-) $F$. filifera & & Base (3.7 Ma) \\
\hline (-) S. membranaceus & & Base (3 Ma) \\
\hline (-) V. calvum & & Base ( $3 \mathrm{Ma})$ \\
\hline (-) Z. lenticulatum & & Base ( $3 \mathrm{Ma})$ \\
\hline (+) O. israelianum & & Base (3 Ma) \\
\hline$\left.{ }^{*}\right)$ I. multiplexum & & Base(3 Ma) \\
\hline Zone III: approximate age: & $2-0.01 \mathrm{Ma}$ & $1.7-0.01 \mathrm{Ma}$ \\
\hline Subzone IIIa: approximate age: & $2-0.95 \mathrm{Ma}$ & $1.7-1.5 \mathrm{Ma}$ \\
\hline \multirow{8}{*}{$\begin{array}{l}(-) \text { F. filifera } \\
(-) \text { I. japonicum } \\
(+) \text { I. velorum } \\
(+) \text { I. strialatum } \\
(+) \text { I. paradoxum } \\
(+) \text { O. israelianum } \\
\text { B. auranteum } \\
(-) \text { Z. lenticulatum }\end{array}$} & Range and top & Top (1.5 Ma) \\
\hline & & Top \\
\hline & Top & \\
\hline & Base & Range \\
\hline & & Top \\
\hline & Base & Top \\
\hline & Base & \\
\hline & Base & \\
\hline Subzone IIIb: approximate age: & $0.95-0.01 \mathrm{Ma}$ & $1.5-0.01 \mathrm{Ma}$ \\
\hline Piperodinium perplexum & Range & Range \\
\hline Glenodinium sp. A & Range & Range \\
\hline Multispinula minuta & Range & Range \\
\hline$(+)$ Votadinium calvum & Range & Top \\
\hline B. auranteum & & Range \\
\hline (*) M. quanta & & Range \\
\hline (+) T. pellitum & & Top \\
\hline (+) S. membranaceus & & Top \\
\hline$\left(^{*}\right)$ S. elongatus & & Range \\
\hline (*) S. frigidus & & Range \\
\hline
\end{tabular}

network. Harland (1977, pp. 103-104, pl. 1, figs. 12-18, text-fig. 4) described a similar taxon from Pleistocene to Recent sediments off Britain, which he called Spiniferites septentrionalis and which he considered might be the same species as $S$. ramuliferus (De- flandre) Reid. I have examined the holotype of Spiniferites septentrionalis and Reid's cyst form, and I agree with Harland (1983), who recently stated that $S$. septentrionalis Harland, 1977 is a junior synonym of $A$. andalousiense, and that it may be reworked in upper Pleistocene sediments along the British coast. I also agree that the specimens of $S$. ramuliferus (Deflandre) Reid belong to the species Achomosphaera ramulifera (Deflandre) Evitt. In my opinion, however, S. ramuliferus is entirely separate from $S$. septentrionalis, because $S$. ramuliferus has much sturdier spines with a relatively small number of distal branches that are not joined to form reticulate complexes.

Achomosphaera ramulifera (Deflandre) Evitt, 1963 (Plate 2, Fig. 3). Reid's material of Spiniferites ramuliferus (Deflandre) Reid, 1974 apparently extends the range top of $A$. ramulifera from middle Miocene (Davey and Williams, 1966) to Recent. The isolated occurrences of $A$. ramulifera in Recent sediments near the coast of Britain (Reid, 1974) suggest, however, that these cysts are reworked. The stratigraphic range of Cenomanian to Pliocene or lower Pleistocene reported by Habib (1972) for A ramulifera appears more likely to be correct.

\section{Genus AMICULOSPHAERA Harland, 1979}

Amiculosphaera umbracula Harland, 1979 (Plate 2, Figs. 5, 8). This may be the same species as Lophocysta sulcolimbata Manum, 1979 according to observations by S. B. Manum (personal communication, June, 1985).

\section{Genus ATAXIODINIUM Reid, 1974}

Ataxiodinium choanum Reid, 1974, pp. 588-589, pl. 1., figs. 1, 2

Genus BITECTATODINIUM Wilson, 1973

Bitectatodinium tepikiense Wilson, 1973

\section{Genus BRIGANTEDINIUM Reid, 1974}

Brigantedinium auranteum Reid, 1974 (Plate 5, Fig. 16). This species is similar to $B$. simplex Reid in its general appearance and archeopyle characteristics. B. auranteum is always distinguishable, however, by its pale brown or yellowish color, and by its hyaline appearance, which indicates the separation between its thick, transparent outer wall and thin, pigmented inner wall. The archeopyle of $B$, auranteum also tends to be more laterally elongated than in $B$. simplex. When compressed, the archeopyle of $B$. auranteum may assume a zig-zag appearance such as that depicted for "?Indet. Protoperidinium cysts" by Harland (1982, pl. 38, figs. 10-12).

Brigantedinium cariacoense Reid, 1974 (Plate 5, Fig. 22)

Brigantedinium simplex Reid, 1974 (Plate 5, Figs. 4, 9)

Brigantedinium spp. (Plate 5, Fig. 20). Numerous specimens with the characteristic brown wall color and the structure of Brigantedinium were found in the Pliocene to Recent samples from Sites 607 and 611. These cysts could not be identified at the species level, because of their variability in shape and wall surface ornamentation, and/or their damaged archeopyle areas; they are therefore, grouped here as Brigantedinium species. One representative form is illustrated in Plate 5, Figure 20.

Genus CANNOSPHAEROPSIS O. Wetzel, 1933, emend. Williams and Downie in Davey et al., 1966

?Cannosphaeropsis sp. 1 (Plate 3, Fig. 6; Plate 5, Fig. 14). This chorate cyst form showed considerable variation in shape, ranging from subrounded (Plate 5, Fig. 14) to ellipsoidal (Plate 3, Fig. 6). The dense ornamentation of branching processes, with spatulate distal branch complexes (Plate 3, Fig. 6) and interconnecting trabeculae, made it impossible to discern clearly the archeopyle and body-wall characteristics. Here this cyst form is tentatively placed in the genus Cannosphaeropsis, but these cysts may belong to the genus Adnatosphaeridium if they have an apical archeopyle. 
Genus CORRUDINIUM Matsuoka, 1983

Corrudinium harlandii Matsuoka, 1983 (Plate 3, Fig. 9a, b). Matsuoka (1983, p. 118) considers that the small proximate cysts described by Harland (1979) as ?Ellipsoidinium sp. can be assigned to $C$. harlandii. Characteristic features of $C$. harlandii are the precingular archeopyle (Plate 3, Fig. 9a), the absence of well-defined tabulation, and the presence of accessory parasutural septa (probably the vermiculate ornamentation referred to by Harland, 1979) within the apparent paraplate boundaries.

\section{Genus FILISPHAERA Bujak, 1984}

Filisphaera filifera Bujak, 1984 (Plate 4, Fig. 6). This species has not previously been reported for Atlantic Ocean sediments, although it is very common in the Pliocene interval of Site 611 and part of the Pliocene of Site 607 . The overall similarity of $F$. filifera to large specimens of Tectatodinium pellitum (e.g., Habib, 1972, pl. 18, fig. 3) suggests that some of the large cyst forms described as $T$. pellitum in Neogene deep-sea sediments from the North Atlantic may in fact be specimens of $F$. filifera.

\section{Genus Glenodinium Ehrenberg, 1837}

Glenodinium sp. A (Plate 5, Figs. 7, 10a, b). This small brown spherical cyst was first reported as Leiosphaera species A by Mudie and Short (1985, pl. 10.1, fig. h), and was described as being abundant in Recent sediments of northwestern Baffin Bay. Examination of plankton tow samples from Baffin Bay fjords later revealed the presence of a small species of Glenodinium which appears to be the thecate stage of these small Arctic cysts (Mudie and Deonarine, 1983). If this affinity can be confirmed by lab culture, then the cyst form should be renamed to indicate its glenodinioid dinoflagellate origin. Specimens from Recent sediments in Baffin Bay (Plate 5, Fig. 7) are slightly smaller and have a thicker wall than those from Sites 607 and 611 (Plate 5, Fig. 10a, b).

\section{Genus HYSTRICHOKOLPOMA Klumpp, 1953; emend. Williams} and Downie in Davey et al., 1966

Hystrichokolpoma sp. 1 (Plate 4, Figs. 10a, b, 11). This is a very small form (maximum width $15-20 \mu \mathrm{m}$ ) with an apical archeopyle and a spherical body bearing three types of processes: (1) relatively large, hollow rectangular processes which are open and fimbriate distally; (2) narrow rod-shaped paracingular processes with blunt apices; and (3) conical parasulcal processes that appear to have solid pointed apices. Scanning electron microscope (SEM) studies are needed to determine the tabulation and morphological details of these small cysts.

Genus HYSTRICHOSPHAEROPSIS Deflandre, 1935, emend. Sarjeant in Davey et al., 1966

Hystrichosphaeropsis obscurus (Habib) Stover and Evitt, 1978. Cysts resembling Hystrichosphaeridium obscurum Habib 1972 were rare in lower Pliocene samples from Site 611. Most specimens were folded and oxidized; therefore, they may be reworked at this site.

\section{Genus IMPAGIDINIUM Stover and Evitt, 1978}

Impagidinium aculeatum (Wall) Lentin and Williams, 1981 (Plate 4, Fig. 4)

Impagidinium aliferum n. sp. (Plate 4, Figs. 1a, b)

Holotype. Plate 4, Fig. 1a, b; slide AGC 84103006, K28/1; Sample 607-23-6, 49-51 cm; GSC 55329, K 28/1.

Derivation of name. Latin ala (wing) and ferro (to bear).

Diagnosis. Cyst proximochorate with an asymmetrical ovoid main body that has an apical boss and widens in the antapical region. The paraplates are outlined by hyaline septa which are highest at the paraplate junctions, where they appear to be supported by thin rods. The septa are lower between the paraplate junctions; however, the septa are high $(>10 \mu \mathrm{m})$ and finely reticulate between paraplates $1^{\prime \prime}$ and $2^{\prime \prime}$ and on the hypotract. An apparently tubular process is formed by septa surrounding the small posterior ventral paraplate. Paratabulation is gonyaulacoid $4^{\prime}, 6^{\prime \prime}, 6 \mathrm{c}, 5^{\prime \prime}, 1 \mathrm{p}, 1^{\prime \prime} \prime^{\prime}, ? 3 \mathrm{~s}$. Excystment is by loss of precingular paraplate $3^{\prime \prime}$.

Dimensions. Holotype: body length $60 \mu \mathrm{m}$, maximum width $50 \mu \mathrm{m}$, septa 13 to $20 \mu \mathrm{m}$ wide. Range in body length 55 to $60 \mu \mathrm{m}$, maximum width 53 to $48 \mu \mathrm{m}$, maximum septum height 14 to $20 \mu \mathrm{m}$. Ten specimens measured.

Description. A large ovoid species of Impaginidium which has a very wide, finely reticulate septum on the epitract between paraplates $1^{\prime \prime}$ and $2^{\prime \prime}$; similar high septa border the posterior parasulcal area, giving the cyst an asymmetrical winged appearance. The parasulcus is very wide and extends far into the epitract, so that paraplates 1' ' and $6^{\prime \prime}$ are very narrow. A small apical boss is present, and there is a tubular process surrounding the posterior ventral platelet. Excystment is by loss of paraplate $3^{\prime \prime}$, which may initially remain attached to the epitract.

Remarks. This species is very similar in morphology to I. aculeatum (Wall) Harland, from which it differs in its much larger size, the presence of an apical boss, reticulate septa, posterior ventral tubular process, and much wider septa that give the cysts an asymmetrical winged appearance. The septal expansion in this species appears to represent a morphological transition between $I$. aculeatum, which has a few, relatively narrow septal expansions, and taxa such as $I$. japonicum and $I$. velorum, which are almost entirely covered by expanded septa.

Occurrence. Upper Miocene to upper Pliocene sediments at Site 611 and lower to upper Pliocene sediments at Site 607.

Impagidinium japonicum Matsuoka, 1983 (Plate 4, Fig. 2a, b). Specimens of this species from Site 607 have less well-defined paracingular paraplates than the cysts from Japan described by Matsuoka (1983, pp. $120-121$, pl. 6, figs. 2-5, text-fig. 13). In this respect, the Site 607 cyst form resembles cysts of 1 . velorum Bujak from the Bering Sea, from which they differ primarily in their narrower, finely granulate, more undulating septa.

Impagidinium multiplexum (Wall and Dale) Lentin and Williams, 1981 (Plate 5, Fig. 15). This species was originally described as Leptodinium multiplexum by Wall and Dale (1968, pp. $318-319$, pl. 1, figs. 1-7, text-fig. 1A-D) from specimens from the Ludham Borehole, England, and it appears to be a good marker of lower Pleistocene sediments in the northeastern Atlantic.

Impagidinium(?) pallidum Bujak 1984 (Plate 3, Fig. 2) This taxon refers to large, thin-walled cysts similar to I. pallidum Bujak (1984, p. 187 , pl. 2, figs. 9-12) from the Bering Sea. At sites 607 and 611 , this cyst form was occasionally found in upper Miocene to Pliocene samples. The tabulation of these cysts and of Bujak's taxon has not yet been determined with certainty, however, so assignment to the genus Impagidinium is presently uncertain.

Impagidinium paradoxum (Wall) Stover and Evitt, 1978 Impagidinium patulum (Wall) Stover and Evitt, 1978 (Plate 4, Fig. 3)

Impagidium sp. A (Plate 4, Fig. 7a, b). This form is probably one of a group of morphologically variable small Impagidinium species $(<30 \mu \mathrm{m})$ that were noted by Edwards (1984) as being abundant in upper Miocene sediments of the Rockall Plateau. Impagidinium sp. A is distinguished from other small Impagidinium species by its relatively wide septa compared with its small body size, by the large width of its paracingular paraplates, and by its wide parasulcus with almost complete development of parasulcal paraplates (only paraplates r.s. and I.s. are incompletely developed or missing). Impaginidium sp. A appears to be similar to I. sphaericum (Wall) Harland, 1984, from which it differs in its much smaller size and wider septa, lack of an apical boss, and incomplete development of parasulcal platelet areas.

Impagidinium sp. B (Plate 4, Fig. 8a, b). This form is another member of the group of small morphologically variable Impagidinium cysts that are abundant in upper Miocene to Pliocene sediments of Sites 607 and 611. Impagidinium sp. B is similar to I. strialatum (Wall) Stover and Evitt, 1978, from which it differs in its much smaller size $(25-30 \mu \mathrm{m})$ and its narrower septa $(<4 \mu \mathrm{m})$, which are not radially striate, and in the presence of one or two apparently complex septal processes on the posterior dorsal surface (possibly at the posterior junctions of paraplates $3^{\prime \prime \prime}$ and $4^{\prime \prime} \prime$, and paraplates $3^{\prime \prime \prime}$ and $2^{\prime \prime \prime}$. 
Impagidinium sp. C (Plate 3, Fig. 14a, b). This form is similar to Impagidinium sp. B, but it is consistently smaller and has a more complete paratabulation, as indicated by the parasutural septa that almost cover the ventral surface (see Plate 4, Fig. 14b). This cyst form is also distinguished by having apparently branched processes at some of the paraplate junctions.

Impagidinium strialatum (Wall) Stover and Evitt, 1978 (Plate 4, Fig. 5)

Impagidinium velorum Bujak, 1984 (Plate 3, Fig. 5a, b)

\section{Genus IMPLETOSPHAERIDIUM Morgenroth, 1966}

Impletosphaeridium spp. (Plate 5, Figs. 12a, b; 13a, b). This category includes small cyst forms that have a spheroidal to ellipsoidal main body bearing solid spines, some of which are branched distally. The main body bears faint, discontinuous septa; a pentagonal excystment aperture could be discerned on some specimens (see Plate 5, Fig. 12a), but the most common form is ellipsoidal without a discernable archeopyle (Plate 5, Figs. 13a, b). SEM studies are required to determine the proper taxonomic affinity of these forms, which may be peridinioid cysts and which probably include more than one species.

\section{Genus INVERTOCYSTA Edwards, 1984}

Invertocysta spp. (Plate 2, Fig. 14). This category includes cysts that could be assigned with certainty to the species Invertocysta lacrymosa Edwards 1984 (Plate 2, Fig. 14) and I. tabulata Edwards 1984, as well as other specimens that were folded or oriented so that species determination was not possible.

\section{Genus LABYRINTHODINIUM Piasecki, 1980}

Labyrinthodinium truncatum Piasecki, 1980 (Plate 3, Figs. 12a, b; 13; Plate 4, Figs. 14a, b). Chorate cysts assigned to this taxon were all characterized by a small size, spherical main body, and a cyst wall ornamented by a closed or open reticulum of anastomosing septa. The wide range of septum development evident in $L$. truncatum (Piasecki, 1980, pl. 6, figs. 2, 3) and the variation in process morphology and paraplate configuration shown by cysts from Site 611 (Plate 3, Figs. 12a, b cf. Fig. 14a, b) suggest that the cyst forms from both the type locality and the North Atlantic include more than one taxon.

\section{Genus LINGULODINIUM Wall, emend. Wall, Dale, and Harada,} 1973

Lingulodinium machaerophorum (Deflandre and Cookson) Wall, 1967 (Plate 5, Fig. 18). Some specimens in samples from Sites 611 and 607 showed excystment by loss of most of the epitractal plates, as described by Wall $(1967$, p. 109 , text-fig. 6C); many specimens, however, were so folded that it was not possible to discern the archeopyle structure, and some showed archeopyles formed by the apparent loss of only two epitractal paraplates, as illustrated in Plate 5, Figure 18.

\section{Genus MULTISPINULA Bradford, 1975}

Multispinula minuta Harland and Reid in Harland et al., 1980 (Plate 5, Fig. 8)

Multispinula quanta Bradford, 1975

Multispinula sp. A (Plate 5, Fig. 19). Specimens of this peridinioid cyst from Site 611 appear to be identical to cysts called ?Protoperidinium sp. A by Mudie and Short (1985, pl. 10.1, fig. f), which are common in Recent sediments of fjord and shelf sediments in northeastern Baffin Bay.

Genus NEMATOSPHAEROPSIS Deflandre and Cookson, 1955, emend. Williams and Downie, 1966

Nematosphaeropsis aquaeducta Piasecki, 1980 (Plate 3, Figs. 7, 11a, b). Cysts described by Piasecki (1980, p. 70, pl. 2, figs. 1-3) as $N$. aquaeducta have relatively large central bodies compared with the lengths of the processes, as shown by the specimens illustrated in
Plate 3, Figure 11. These characteristics are also found in Leptodinium sp. V of Manum (1976, pl. 1, figs. 19-20), which Piasecki (1980) considers to be the same taxon, but Manum (personal communication, June, 1985) does not agree with this synonymy, and he considers that the specimens in Plate 3, Fig. 11 are probably an Impagidinium species. Samples from Sites 607 and 611 frequently also contain a Nematosphaeropsis-like cyst form (Plate 3, Fig. 7) with a much smaller main body and relatively larger, stouter processes. This form also conforms to Piasecki's description of $N$. aquaeducta, and has been included with this taxon for this report; but Manum disagrees with this assignment, and further studies must be made of the apparently wide range of morphological variation in cyst forms that have been referred to $N$. aquaeducta.

Nematosphaeropsis oblonga n. sp. (Plate 3, Figure 4a, b)

Holotype. Plate 3, Figure 4a, b; slide AGC 84031903, Z 55/1; Sample 611C-35-3, 48-50 cm; GSC 55318, Z 55/1.

Derivation of name. Latin oblonga (elongate).

Diagnosis. Cyst chorate, with a characteristically ellipsoidal central body, for which the length is at least 2 times longer than the width. The body bears numerous sturdy gonal processes at least as long as the body width. The processes terminate in short trifurcate distal branches, which are joined by double trabeculae and form undulating parallel lines. The archeopyle seems to be formed by loss of paraplate $3^{\prime \prime}$.

Dimensions. Holotype: body length $30 \mu \mathrm{m}$, width $15 \mu \mathrm{m}$, gonal spine length 12 to $17 \mu \mathrm{m}$. Range in body length 35 to $28 \mu \mathrm{m}$, width 12.5 to $15 \mu \mathrm{m}$, spine length 12 to $25 \mu \mathrm{m}$. Ten specimens measured.

Description. A relatively large ellipsoidal cyst of Nematosphaeropsis whose distinctive elongate central body bears sturdy gonal spines with trifurcate branch tips interconnected by trabeculae. The longest trabeculae form undulating parallel lines, which appear to reflect parasutural boundaries. The archeopyle is trapezoidal and formed by loss of a single paracingular plate, presumably paraplate $3^{\prime \prime}$. Tabulation cannot be clearly discerned, but the number of paraplates delimited by the trabeculae is about the same as in N. labyrinthea.

Remarks. $N$. oblonga has the overall appearance of a $N$. labyrinthea cyst that has been compressed. However, numerous specimens of this ellipsoidal taxon coexist with normal spherical forms of $N$. labyrinthea; in these samples, the specimens of $N$. oblonga do not appear to be folded, damaged, or poorly preserved, and the spines are not broken or bent as would be expected if these cyst were merely poorly preserved forms of $N$. labyrinthea. The consistency of the elliptical body shape and morphology of the trabeculae processes, and the coexistance of these ellipsoidal cysts with typical spherical cysts of $N$. labyrinthea in sediments of late Miocene to early Pliocene age, makes $N$. oblonga a cyst form worthy of specific taxonomic differentiation.

Nematosphaeropsis labyrinthea (Ostenfeld) Reid, 1974 (Plate 3, Fig. 1a, b)

Nematosphaeropsis sp. 1 (Plate 3, Fig. 3). These cyst forms have an appearance generally similar to that of $N$. labyrinthea, but they differ in the following ways: the main body has a much thinner wall, so that it is impossible to discern the archeopyle characteristics using light microscopy; the trabeculae form short pairs of ribbonlike strands only in restricted areas of the periphragm around the process tips, and the longer ?parasutral trabeculae are single strands; some of the short trabeculae at the process tips are strongly arched, forming vermiculate complexes along with the short parallel strands. The small, thin-walled main body and short arched trabeculae of Nematosphaeropsis sp. 1 resemble these features of the paratype of N. lemniscata Bujak, 1984, from the Bering Sea (see Bujak's pl. 3, fig. 6), but Nematosphaeropsis sp. 1 lacks the long parallel ribbonlike trabeculae. of $N$. lemniscata.

\section{Genus OPERCULODINIUM Wall, 1967}

Operculodinium centrocarpum (Deflandre and Cookson) Wall, 1967 (Plate 2, Fig. 4). See remarks that follow concerning the differences between $O$. centrocarpum, ?O. echigoense, and $O$. longispinigerum. Also, it is clear that specimens from the Tabiano stratotype which Habib (1971) called O. centrocarpum are more similar to $O$. wallii and $O$. echigoense than to Wall's illustrations of $O$. centrocarpum.

?Operculodinium echigoense Matsuoka, 1983 (Plate 2, Fig. 7). As described by Matsuoka (1983), this species is distinguished from $O$. 
centrocarpum by a finely reticulate or granular wall surface and relatively long, slender, hollow processes; illustrations of the paratypes (Matsuoka, 1983, pl. 7, figs. 1-5) show, however, that the processes are relatively short compared with the main body diameter, and they appear to be structurally weak, hence often vermiculate in appearance and frequently folded. All cysts from Sites 611 and 607 that were assigned to this taxon have apparently weak spines (see Plate 2, Fig. 7), and are easily distinguished from $O$. centrocarpum, which normally has erect straight spines (see Plate 2, Fig. 4). Matsuoka (1983) gives the range of $O$. echigoense as late early Miocene to late Miocene or younger.

Operculodinium crassum Harland, 1979 (Plate 2, Figure 9; Plate 5, Fig. 6)

Operculodinium israelianum (Rossignol) Wall, 1967

Operculodinium longispinigerum Matsuoka, 1983 (Plate 2, Fig. 13). Matsuoka (1983, p. 125) commented that this taxon is distinguished from $O$. centrocarpum by its smaller number of process, which have acuminate or bifurcate rather than capitate distal extremities. In my experience, however, specimens of $O$. centrocarpum in Pleistocene sediments of the North Atlantic (including specimens described by Wall, 1967; Reid, 1974; and Harland, 1977) always have slightly expanded distal process endings that are minutely bifurcate. Cysts of $O$. longispinigerum from Sites 607 and 611 also have bifurcate process tips, and they have only one-third to one-half as many processes as $O$. centrocarpum; these processes always have a somewhat crumpled appearance, and are usually doubled over toward the central body, as shown in Plate 2, Figure 13, and in illustrations of the holotype (Matsuoka, 1983, pl. 9, fig. 8a, b).

Operculodunium psilatum Wall, 1967

?Operculodinium sp. 1 (Plate 4, Fig. 9a, b). This form superficially resembles Operculodinium sp. of Jan du Chêne (1977) (see Plate 2, Fig. 12a, b), from which it is distinguished by its larger size (50$55 \mu \mathrm{m})$ and its minutely verrucate wall surface, and by the consistent presence of a conspicuous rhomboidal or hexagonal paraplate or thickened wall area that appears to be present on the ventral surface (Plate 4, Fig. 9b). In dorsal? view, the archeopyle appears to be hexagonal in outline (Plate 4, Fig. 9a). and it appears that the operculum remains attached to the anterior margin of the archeopyle after excystment and becomes folded inward against the cyst wall. SEM studies are needed to clarify the nature of this form, which may be a peridinioid species.

Operculodinium sp. of Jan du Chêne (1977) (Plate 2, Fig. 12a, b). Cysts assigned to this taxon are always small (maximum diameter $40 \mu \mathrm{m})$ with a microgranular wall surface and very short $(<4 \mu \mathrm{m})$ processes, which are conical with open ends, as described by Jan du Chêne (1977, p. 108); the specimens from Sites 611 and 607 differ, however, from Jan du Chêne illustrations in having numerous solid conical granules or very short solid spines interspersed between the tubular processes.

Operculodinium sp. of Piasecki (1980) (Plate 2, Fig. 11a, b)

Operculodinium wallii Matsuoka, 1983 (Plate 2, Fig. 1). This taxon resembles $O$. centrocarpum but has a much larger central body (>50 $\mu \mathrm{m})$ and longer processes $(12-20 \mu \mathrm{m})$ which are more flexuous and are recurved at the distal ends. Matsuoka $(1983$, p. 127) gives a late Miocene to early Pliocene range for his species. Habib (1971, pl. 1, fig. 4) illustrates a large cyst from the Tabiano stratotype which he called $O$. centrocarpum; this specimen has the typical process characteristics of $O$. wallii and is probably conspecific with it.

\section{Genus PALAEOSTOMOCYSTIS Deflandre, 1935}

Palaeostomocystis spp. of Baltes (1971) (Plate 5, Fig. 21). Two large spherical Palaeostomocystis species with a granular or microgranular wall surface and wide apical excystment aperture were described by Baltes (1971) as Palaeostomocystis sp. I and Palaeostomocystis sp. II. Similar forms were found in samples from Sites 611 and 607 , but their morphological variability precluded definite assignment to Baltes' species; therefore, they were grouped together as Palaeostomocystis spp. One representative specimen with a granular wall surface is shown in Plate 5, Figure 21; other cyst forms al- so included in this category had thinner smooth, brownish walls without any discernible ornamentation or tabulation.

\section{Genus PIPERODINIUM Mudie in Scott et al., 1984}

Remarks. A description of this genus and its type species, Piperodinium perplexum, was given in Latin by Mudie (in Scott et al., 1984, pp. 214-215, pl. 1, p. 192, figs. 1-3). An English translation is given here.

Derivation of name. Latin Piperi, in honor of the Canadian geologist David J. W. Piper.

Description. Ellipsoidal cysts, with a smooth interior wall; apices rounded before excystment; and outer cyst wall with very short spines delimiting part of the paracingulum and other paraplate margins; these spines or processes are linked by low septa. Excystment appears to occur in the apical area because of loss of a single paraplate.

Type species. Piperodinium perplexum Mudie, n. sp.

Piperodinium perplexum Mudie in Scott et al., 1984 (Plate 5, Fig. 11)

Derivation of name. Latin perplexo (to entangle, bewilder) with reference to the nature of the outer wall ornamentation and the nature of the archeopyle.

Holotype. Scott et al., 1984, pl. 1 (p. 192), figs. 1-3; slide PJM 78005-95, 0 cm; England Finder coordinates U 30/2; GSC 55369, U 30/2; Sample 78-005-95, 0-1 cm, from the upper slope of the Nova Scotian Shelf.

Description. Cyst ellipsoidal and small (about $35 \times 50 \mu \mathrm{m}$ to $50 \times$ $40 \mu \mathrm{m}$ for 10 cysts); the inner wall is thick and colorless; usually without internal bodies but rarely containing red ?oil bodies. The outer wall usually bears 20 to 30 short solid spines $(<2 \mu \mathrm{m})$ and 15 longer conical spines $(5 \mu \mathrm{m})$, which support ?parasutural septa. The paracingulum and other paraplate outlines are often discontinuous. Most of the cysts lack an archeopyle; rare cysts have an archeopyle in the apical area, with the operculum attached to one side.

Remarks. Cysts of $P$. perplexum from Sites 607 and 611 are similar to the holotype described from Recent sediments on the Scotian Slope, but the archeopyle in some specimens appears to be intercalary rather than apical (see Plate 5, Fig. 11). This suggests that more than one paraplate may be displaced during excystment; as in the holotype, however, the operculum remains attached to the margin of the archeopyle.

\section{Genus PLATYCYSTIDIA Cookson and Eisenack, 1960}

Platycystidia(?) sp. 1 of Manum (1976)

Genus POLYSPHAERIDIUM Davey and Williams, 1966, emend. Bujak et al., 1980

Polysphaeridium zoharyi (Rossignol) Bujak et al., 1980

Remarks. This species was formerly known as Hemicystodinium zoharyi (Rossignol) Wall, 1967 and Hystrichosphaeridium zoharyi Rosignol 1962.

\section{Genus PYXIDIELLA Cookson and Eisenack, 1958}

Pyxidiella sp. 1 (Plate 4, Fig. 12a, b). This small cyst $(<30 \mu \mathrm{m})$ has a large subcircular pylome or apical archeopyle (Plate 4, Fig. 12a) and a small antapical horn that protrudes toward the ventral surface. SEM studies are needed to determine whether this dinoflagellate is a peridinioid cyst.

Genus SELENOPEMPHIX Benedek, 1972, emend. Bujak, 1980

Selenopemphix nephroides Benedek, 1972, emend. Bujak, 1980 (Plate 5, Fig. 1)

Genus SPINIFERITES Mantell, 1950, emend. Sarjeant, 1970

Spiniferites bentori (Rossignol) Wall and Dale, 1970 Spiniferites elongatus Reid 1974 (Plate 4, Fig. 15)

Spiniferites frigidus Harland and Reid in Harland et al., 1980. Specimens of $S$. frigidus from Site 611 are almost identical in appear- 
ance to cyst forms from Baffin Bay illustrated by Mudie and Short (1985, pl. 10.1, fig. c).

Spiniferites membranaceus (Rossignol) Sarjeant, 1970

Spiniferites mirabilis (Rossignol) Sarjeant, 1970 (Plate 2, Fig. 10)

Spiniferites cf. pseudofurcatus (Klumpp) Sarjeant, 1970. Specimens in samples from Sites 611 and 607 that were referred to this taxon are similar to those described and illustrated by Harland (1979, pp. $536-537$, pl. 2, figs. 19, 20).

?Spiniferites splendidus Harland 1979 (Plate 4, Fig. 13). Most specimens in samples from Sites 611 and 607 that were referred to this taxon had less distinct parasutural ridges than those present in cysts described by Harland $(1979$, p. 537 , pl. 3, figs. 1, 2), and it is possible that the specimens from Sites 611 and 607 should be assigned to the genus Achomosphaera.

Spiniferites spp. (Plate 2, Fig. 2; Plate 5, Fig. 5). This category refers to a group of Spiniferites cysts that were common in upper Miocene samples from Sites 611 and 607 and which are characterized by relatively short, weakly developed spines. These cysts showed a wide variation in surface ornamentation of the cyst body, in the structure of the processes, and in their distal endings. The specimens illustrated in Plate 2, Figure 2 and Plate 5, Figure 5 demonstrate the range of variation in these cyst forms which have been grouped together as Spiniferites spp.

\section{Genus TECTATODINIUM Wall, 1967}

Tectatodinium pellitum Wall, 1967

Tectatodinium simplex (Harland) Edwards, 1984 (Plate 3, Figs. 8a, b, 10). Edwards (1984) states that the following late Miocene cyst forms are conspecific with $T$. simplex (Harland) Edwards: ?Pyxidiella simplex Harland 1979; specimens referred to as Tectatodinium psilatum Wall and Dale 1973 by Piasecki (1980, pl. 4, figs. 2, 3). In my opinion, T. simplex (Harland) Edwards also includes Pyxidiella sp. of Habib (1971, p. 492, pl. 4, fig. 1), which was considered to be a potential guide fossil for the Piacenzian Stage of the Tabiano stratotype. At Sites 607 and 611, small cysts morphologically intermediate between T. simplex (Plate 3, Fig. 10), ?Pyxidiella scrobiculata Harland 1979 (Plate 3, Fig. 8a, b), and Pyxidiella sp. 1 of Mudie (pl. 4, fig. 12) were also found. This group of taxa clearly requires more detailed study.

\section{Genus TUBERCULODINIUM Wall, 1967, emend. Wall and Dale,} 1971

Tuberculodinium vancampoae (Rossignol) Wall, 1967 (Plate 5, Fig. 17)

\section{Genus VOTADINIUM Reid, 1977}

Votadinium calvum Reid, 1977 (Plate 5, Fig. 2)

\section{Genus ZYGABIKODINIUM Loeblich and Loeblich, 1970}

Zygabikodinium lenticulatum Wall and Dale, 1968, emend. Bujak and Davies, 1983 (Plate 5, Fig. 3). This taxon refers to peridinioid cysts resembling cyst forms of Diplopeltopsis minor described by Wall and Dale $(1968$, p. 280 , pl. 4 , figs. 21,22 ; text-fig. 7$)$ and later described as Dubridinium caperatum by Reid $(1977$, p. 451$)$. Bujak and Davies (1983, p. 47) note that the correct generic name for Diplopeltopsis minor is Zygabikodinium lenticulatum.

Incertae sedis sp. I of Edwards (1984) (Plate 4, Fig. 16). This form comprises very large cysts with an ovoid main body bearing six very long $(27-60 \mu \mathrm{m})$ blunt-tipped processes which are triangular in cross-section. Edwards (1984) found one cyst with a five-sided archeopyle, but most specimens appear to have an epitractal archeopyle, as shown in Plate 4, Fig. 16.

\section{ACKNOWLEDGMENTS}

I thank Philip Hill of the Leg 94 shipboard party for enabling me to obtain the DSDP samples used for this study; Larry Ogden, Dalhousie University, for processing the samples; and Lucy Edwards, USGS, Reston, for making her DSDP Leg 81 data available to me in 1983. Robert Fensome and Jean Dabros, GSC, Atlantic Geoscience
Centre, Svein B. Manum, University of Oslo, Jonathan Bujak, Bujak Research Ltd., Calgary, and Rex Harland, British Geological Survey, reviewed this paper.

\section{REFERENCES}

Aksu, A. E., and Mudie, P. J., 1985. Magnetostratigraphy and palynology demonstrate at least 4 million years of Arctic Ocean sedimentation. Nature, 318:280-283.

Baltes, N., 1971. Pliocene Dinoflagellata and Acritarcha in Romania. In Farinacci, A. (Ed.), Proc. Second Planktonic Conf. (Rome, 1970) (Vol. 1): Rome (Edizioni Technoscienza), 1-16.

Benedek, P. N., 1972. Phytoplanktonen aus dem Mittel- und Oberoligozän von Tönisberg (Niederrheingebiet). Palaeontographica (Abt. B), 137:1-71.

Berggren, W. A., 1981. Correlation of Atlantic, Mediterranean, and Indo-Pacific Neogene stratigraphies: Geochronology and chronostratigraphy. IGCP Project 114; Int. Workshop Pacific Neogene Biostratigr. (Osaka and Kobe, Japan, Nov. 24-29), pp. 93-110.

Bradford, M. R., 1975. New dinoflagellate cyst genera from the Recent sediments of the Persian Gulf. Can. J. Botany, 53:3064-3074.

Bujak, J. P., 1980. Dinoflagellate cysts and acritarchs from the Eocene Barton Beds of southern England. In Bujak, J. P., Downie, C., Eaton, G. L., and Williams, G. L. (Eds.), Dinoflagellate Cysts and Acritarchs from the Eocene of Southern England. Palaeontol. Assoc. Spec. Pap. Palaeontol., 24:2-91.

1984. Cenozoic dinoflagellate cysts and acritarchs from the Bering Sea and northern North Pacific, DSDP Leg 19. Micropaleontology, 30:180-212.

Bujak, J. P., Barss, M. S., and Williams, G. L., 1977. Offshore East Canada's organic type and color and hydrocarbon potential. Oil Gas J., 75 (April 4, 1977): 198-202.

Bujak, J. P., and Davies, E. H., 1983. Modern and fossil Peridiniineae. Am. Assoc. Strat. Palynol. Contr., Ser. 13.

Bujak, J. P., Downie, C., Eaton, G. L., and Williams, G. L., 1980. Dinoflagellate cysts and acritarchs from the Eocene of southern England. Palaeontology Spec. Pap., 24:1-100.

Cookson, I. C., and Eisenack, A., 1958. Microplankton from Australian and New Guinea upper Mesozoic Sediments. Proc. R. Soc. Victoria, 70:19-79.

1960. Microplankton from Australian Cretaceous sediments. Micropaleontology, 6:1-18.

Costa, L. I., and Downie, C., 1979. Cenozoic dinocyst stratigraphy of Sites 403 to 406 (Rockall Plateau, IPOD, Leg 48. In Montadert, L., Roberts, D. G., et al., Init. Repts. DSDP, 48: Washington (U.S. Govt. Printing Office), 513-529.

Davey, R. J., and Williams, G. L., 1966. The genera Hystrichosphaera and Achomosphaera. In Davey, R. J., Downie, C., Sarjeant, W. A. S., and Williams, G. L. (Eds.), Studies on Mesozoic and Cainozoic Dinoflagellate Cysts. Bull. British Mus. (Nat. Hist). Geol., Suppl. $3: 28-52$.

Deflandre, G., 1935. Considérations biologiques sur les microorganisms d'origine planctonique conservés dans les silex de la craie. Bull. Biol. France Belgique, 69:213-244.

Deflandre, G., and Cookson, I. C., 1955. Fossil microplankton from Australian late Mesozoic and Tertiary sediments. Austral. J. Mar. Freshwater Res. 6:242-313, pl. 1-9.

Edwards, L. E., 1984. Miocene dinocysts from Deep Sea Drilling Project Leg 81, Rockall Plateau, eastern North Atlantic Ocean. In Roberts, D. G., Schnitker, D., et al., Init. Repts. DSDP, 81: Washington (U.S. Govt. Printing Office), 581-594.

Ehrenberg, C. G., 1837. Zusätze zur Erkenntnis grosser organischer Ausbildung in den kleinsten thierischen Organismen. Abh. Preuss. Akad. Wiss. 1835, pp. 151-180.

Evitt, W. R., 1963. A discussion and proposals concerning fossil dinoflagellates, hystrichospheres, and acritarchs, I. Proc. Nat. Acad. Sci. U.S., 49:158-164.

Fritsch, F. E., 1929. Evolutionary sequence and affinities among Protophyta. Biol. Rev., 4:103-151.

Habib, D., 1971. Dinoflagellate stratigraphy across the Miocene-Pliocene boundary, Tabiano stratotype section. In Farinacci, A. (Ed.), Proc. Second Planktonic Conf. (Rome, 1970) (Vol. 1): Rome (Edizioni Technoscienza), 591-598.

1972. Dinoflagellate stratigraphy, Leg 11, Deep Sea Drilling Project. In Hollister, C. D., Ewing, J. I. et al., Init. Repts. DSDP, 11: Washington (U.S. Govt. Printing Office), 367-425. 
1979. Sedimentary origin of North Atlantic Cretaceous palynofacies. In Talwani, M., Hay, W. W., and Ryan, W. B. F. (Eds.), Results of Deep Drilling in the Atlantic. Proc. Second Maurice Ewing Symp. (Am. Geophys. Union), pp. 420-437.

Haeckel, E., 1884. Entwurf eines natürlichen Systems de Organismen auf Grund ihrer Stammegeschichte Erster Teil. Systematische Phylogenie der Protiste und Pflanzen: Berlin (Georg Reimer).

Harland, R., 1977. Recent and late Quaternary (Flandrian and Devensian) dinoflagellate cysts from marine continental shelf sediments around the British Isles. Palaeontographica (Abt. B.), 164:87-126. 1978. Quaternary and Neogene dinoflagellate cysts. In Thusu, B. (Ed.), Distribution of Biostratigraphically Diagnostic Dinoflagellate Cysts and Miospores for the Northwest European Continental Shelf and Adjacent Areas. Continental Shelf Inst. Publ. 100:7-17.

1979. Dinoflagellate biostratigraphy of Neogene and Quaternary sediments at Holes $400 / 400 \mathrm{~A}$ in the Bay of Biscay (Deep Sea Drilling Project Leg 48). In Montadert, L., Roberts, D. G., et al., Init. Repts. DSDP, 48: Washington (U.S. Govt. Printing Office), 531-545.

1982. A review of Recent and Quaternary organic-walled dinoflagellate cysts of the genus Protoperidinium. Palaeontology, 25:369-397.

1983. Distribution maps of recent dinoflagellate cysts in bottom sediments from the North Atlantic Ocean and adjacent seas. Palaeontology, 26:321-387.

Harland, R., Reid, P. C., Dobell, P., and Norris, G., 1980. Recent and sub-Recent dinoflagellate cysts from the Beaufort Sea, Canadian Arctic. Grana, 9:211-225.

Harland, W. B., Cox, A. V., Llewellyn, P. G., Pickton, C. A. G., Smith, A. C. T., and Walters, R., 1982. A Geologic Time Scale: Cambridge (Cambridge University Press).

Jan du Chêne, R., 1977. Etude palynologique du Miocene supérieur Andalou (Espagne). Rev. Espan. Micropaleontol., 9:97-114.

Lentin, J. K., and Williams, G. L., 1981. Fossil Dinoflagellates: Index to Genera and Species, (1981 edition). Bedford Inst. Oceanogr. Rept. Ser. BI-R-81-12/August 1981.

Loeblich, A. R., Jr., and Loeblich A. R. III, 1970. Index to the genera, subgenera, and sections of the Pyrrhophyta, IV. J. Paleontol., 44:536-543.

Manum, S. B., 1976. Dinocysts in Tertiary Norwegian-Greenland Sea sediments (Deep Sea Drilling Project Leg 38), with observations on palynomorphs and palynodebris in relation to environment. In Talwani, M., Udintsev, G., et al., Init. Repts. DSDP, 38: Washington (U.S. Govt. Printing Office), 897-919.

1979. Two new Tertiary dinocyst genera from the Norwegian sea: Lophocysta and Evittosphaerula. Rev. Palaeobot. Palynol., 28:237-248.

Matsuoka, K., 1983. Late Cenozoic dinoflagellates and acritarchs in the Niigata District, Central Japan. Palaeontographica (Abt. B), $187: 89-154$.

Morgenroth, P., 1966. Mikrofossilien und Konkretionen des nordwesteuropäischen Untereozäns. Paleontographica (Abt. B), 119:1-53.

Mudie, P. J., 1980. Palynology of late Quaternary marine sediments, eastern Canada [Ph.D thesis]. Dalhousie University, Halifax, Nova Scotia.

1985. Palynology of the CESAR cores, Alpha Ridge. In Jackson, H. R., Mudie, P. J., and Blasco, S. M. (Eds.), Initial Geological Report on CESAR-the Canadian Expedition to Study the Alpha Ridge, Arctic Ocean. Geol. Surv. Pap. (Geol. Surv. Can.), 84-22, Ch. 11.

Mudie, P. J., and Deonarine, B., 1983. Dinoflagellates and pollen from plankton tows and surface sediments, Baffin Island fjords. In Syvitski, J. M., and Blakeney, C. (Eds.), SAFE Initial Data Report (Vol.
1), pp. 20-1 to 20-7; Canadian Data Report of Hydrography and Ocean Series (No. 12), pp. 20-1 to 20-7.

Mudie, P. J., and Short, S. K., 1985. Marine palynology of Baffin Bay. In Andrews, J. T. (Ed.), Quaternary Environments: Eastern Canadian Arctic, Baffin Bay and West Greenland: London (George Allen and Unwin Ltd.), pp. 263-308.

Pascher, A., 1914. Über Flagellaten und Algen. Deutsch. Bot. Gesell. Ber., 36:136-160.

Perconig, E., 1964. La estratigráfia del Mioceno en Andalucia occidental (España). El limite Ogligoceno-Mioceno y la fase terminal marina del Mioceno. Cursillos Conf. Inst. "Lucas Mallada" Est. Geol., 9:219-228.

1973. El estratotipo del Andaluciense. In Perconig, E., and Granados, L. F. (Eds.), Livret guide du XIII européen de Micropaléontologie: Lyon, France (Comité Mediterranean de Stratigraphie du Néogène), pp. 247-251.

Piasecki, S., 1980. Dinoflagellate cyst stratigraphy of the Miocene Hodde and Gram formations, Denmark. Bull. Geol. Soc. Denmark, 29:53-76.

Reid, P. C., 1974. Gonyaulacacean dinoflagellate cysts from the British Isles. Nova Hedwigia, 25:579-637.

1977. Peridiniacean and Glenodiniacean dinoflagellate cysts from the British Isles. Nova Hedwigia, 29:429-462.

Rossignol, M., 1962. Analyse pollinique de sédiments marins Quaternaires en Israel. II. Sédiments Pleistocènes. Pollen et Spores, 4: 121-148.

Sarjeant, W. A. S., 1970. The genus Spiniferites Mantell, 1950 (Dinophyceae). Grana, 10:74-78.

Scott, D. B., Mudie, P. J., Vilks, G., and Younger, D. C., 1984. Latest Pleistocene-Holocene paleoceanographic trends on the continental margin of eastern Canada: Foraminiferal, dinoflagellate and pollen evidence. Mar. Micropaleontol., 9:181-218.

Stockmarr, J., 1971. Tablets with spores used in absolute pollen analysis. Pollen et Spores, 13:615-621.

Stover, L. E., and Evitt, W. R., 1978. Analyses of pre-Pleistocene organic-walled dinoflagellates Stanford Univ. Publications, Geol. Sci., 15:1-300.

Wall, D., 1967. Fossil microplankton in deep-sea cores from the Caribbean Sea. Palaeontology, 10:95-103.

Wall, D., and Dale, B., 1968. Early Pleistocene dinoflagellates from the Royal Society borehole at Ludham, Norfolk. New Phytol., 67: 315-326.

1970. Living hystrichosphaerid dinoflagellate spores from Bermuda and Puerto Rico. Micropaleontology, 16:47-58.

1971. A reconsideration of living and fossil Pyrophycus Stein, 1883 (Dinophyceae). J. Phycol., 7:221-235.

Wall, D., Dale, B., and Harada, K., 1973. Descriptions of new fossil dinoflagellates from the late Quaternary of the Black Sea. Micropaleontology, 19:18-31.

Williams, G. L., 1975. Dinoflagellate and spore stratigraphy of the Mesozoic-Cenozoic, offshore eastern Canada. Geol. Surv. Pap. (Geol. Surv. Can.), 74-30 (Vol. 2):107-161.

Williams, G. L., and Downie, C., 1966. Further dinoflagellate cysts from the London Clay. In Davey, R. J., Downie, C., Sarjeant, W. A. S., and Williams, G. L. (Eds.), Studies on Mesozoic and Cainozoic Dinoflagellate Cysts. Bull. British Mus. (Nat. Hist.) Geol. Suppl. 3:215-235.

Wilson, G. J., 1973. Palynology of the middle Pleistocene Te Piki bed, Cape Runaway, New Zealand. New Zealand J. Geol. Geophys., $16: 345-354$.

Date of Initial Receipt: 26 March 1985

Date of Acceptance: 29 May 1985 

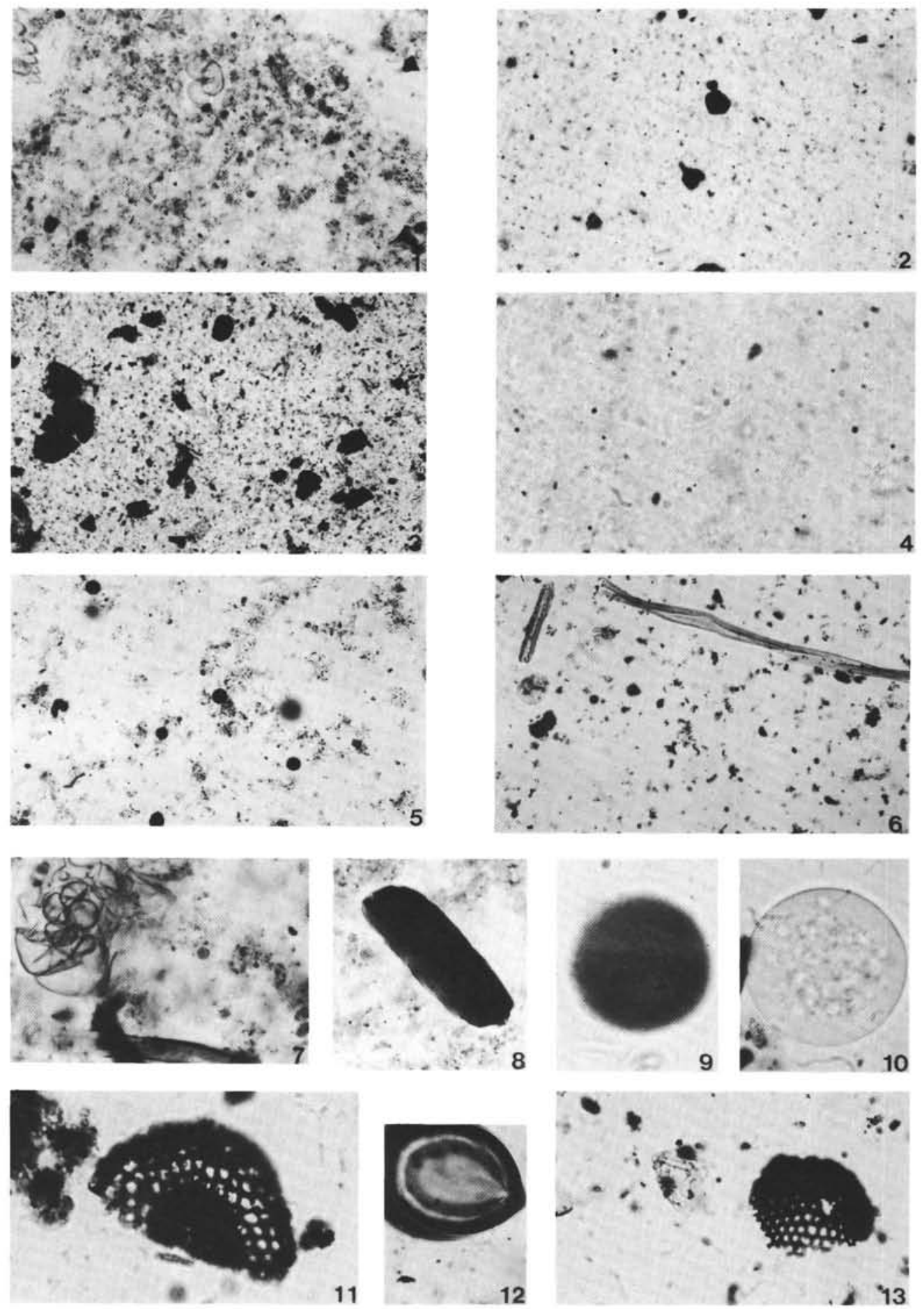

Plate 1. (Magnification is $\times 110$ for Figs. $1-6 ; \times 300$ for Figs. 7, 8, and 11-13; $\times 700$ for Figs. 10, 11. All photographs taken in normal transmitted light.) 1,7,8. Sample 607-1-1, 49-51 cm, (1) abundant amorphogen, typical of Pleistocene interglacial sediment, AGC 85022501, (7) chitinous foraminiferal lining, AGC 85022503, (8) brown wood fragment, AGC 85022502. 2. Abundant, finely divided mineral detritus and poorly sorted carbonized (coaly) particles, typical of Pleistocene glacial sediment, AGC 85022504 , Sample $611-2-1,58-60 \mathrm{~cm}$. 3. Abundant, poorly sorted carbonized organic fragments, AGC 8502222 , Sample $611-8-4,48-50 \mathrm{~cm}$. 4. Abundant silt- to clay-sized mineral grains and frequent coaly particles typical of Pliocene glacial sediments, Site 611, AGC 84110110 , Sample 611C-17-1, 38-40 cm. 5. Amorphogen flocs with finely divided pyrite and abundant black (pyritized) spores, typical of upper Miocene-lower Pliocene sediments, AGC 8502218, Sample 611C-41-5, 92-94 cm. 6. Well-dispersed amorphogen and pyrite, with common translucent plant fibers, AGC 8502221, Sample 611C-44-1, 61-63 cm. 9. Black algal spore, AGC 84103013, S30/3, Sample 607-9-1, 49-51 cm. 10. Translucent algal spore, AGC 85022505 , Sample 611-29-5, 48-50 cm. 11, 13. Sample 607-12-6, 49-51 cm, (11) pyritized diatom, AGC 84103025, (13) diatom fragment and pyrite flocs, AGC 84103024, R48/4. 12. Microtectite, AGC 85012402, Sample 607-23-4, 49-51 cm. 

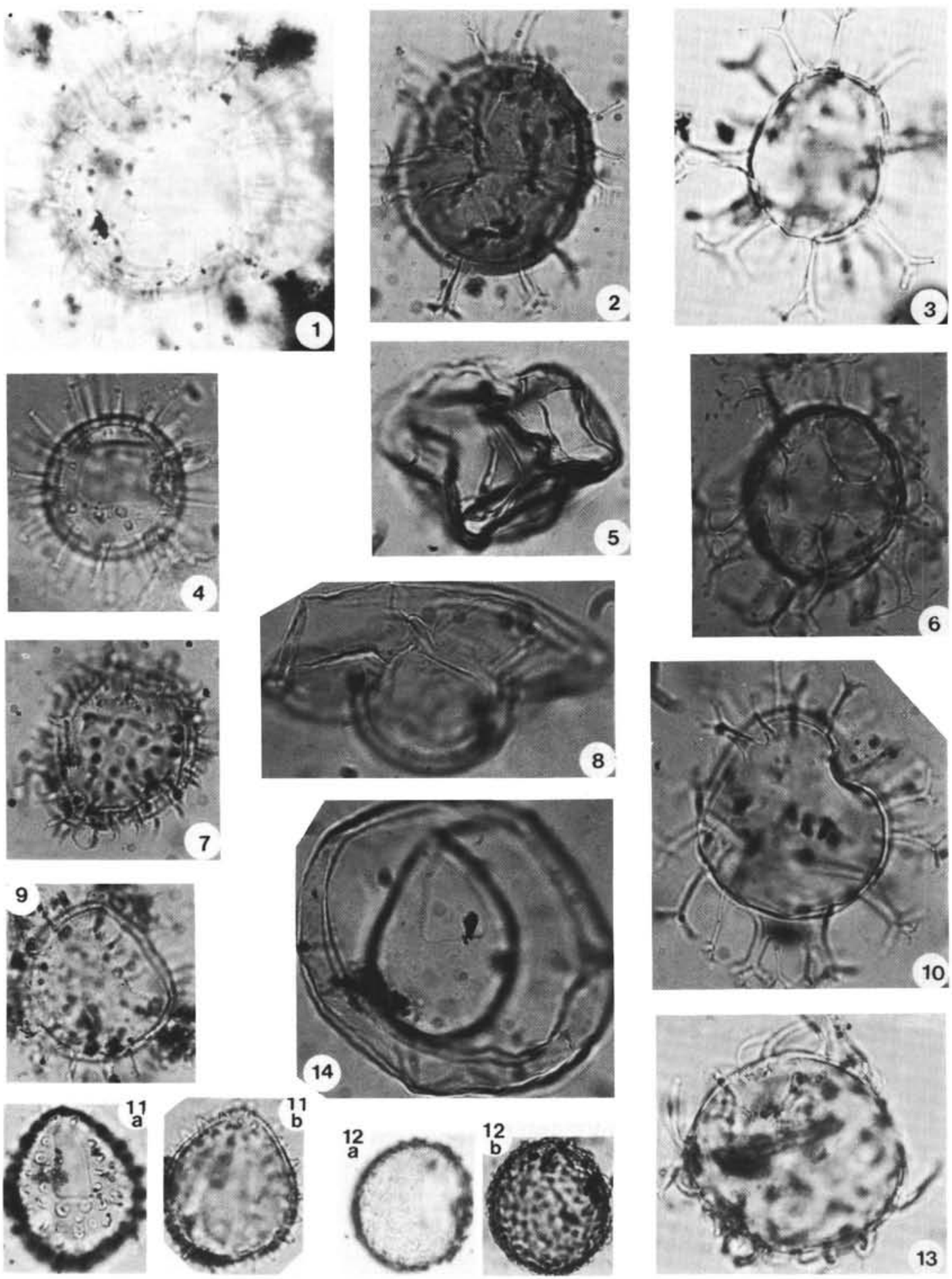

Plate 2. (Magnification $\times 700$, except Fig. 12a, b which is $\times 300$; all figures photographed in normal transmitted light.) 1, 13. Sample 607-27-4, 49-51 cm, (1) Operculodinium wallii Matsuoka, 1983, oblique dorsal view, GSC 55302, W15/3, (13) Operculodinium longispinigerum Matsuoka, 1983, optical section, GSC 55314, Y24/2 2 2, 7. Sample 611C-47-6, 38-40 cm, (2) Spiniferites sp., optical section, GSC 55310, P39/4, (7) ?Operculodinium echigoense Matsuoka, 1983, optical section, GSC 55308, N42/1. 3, 5. Sample 607-27-1, 49-51 cm, (3) Achomosphaera ramulifera (Deflandre) Evitt, 1963, optical section, GSC 55304, P34/4, (5) Amiculosphaera umbracula Harland, 1979, apical view, with archeopyle at upper right, GSC 55303, V39/3. 4. Operculodinium centrocarpum (Deflandre and Cookson) Wall, 1967, dorsal view, GSC 55305, H42/4, Sample 611-14-2, 48-50 cm. 6. Achomosphaera andalousiense Jan du Chêne, 1977, optical section, GSC 55307, T13/2, Sample 611C-45-1, 79$81 \mathrm{~cm}$. 8, 14. Sample 607-23-6, 49-51 cm, (8) Amiculosphaera umbracula Harland, 1979, lateral view showing expanded epitract and apical process, GSC 55366, J 33/3, (14) Invertocysta lacrymosa Edwards, 1984, dorsal view, with archeopyle in focus, GSC 55306, G24/0. 9. Operculodinium crassum Harland, 1979, optical section, with archeopyle at upper right, GSC 55309, J49/3, Sample 611C-24-4, 48-50 cm. 10. Spiniferites mirabilis (Rossignol). Sarjeant, 1970, optical section, GSC 55311, M30/0, Sample 607-16-1, 49-51 cm. 11. Operculodinium sp. of Piasecki (1980), GSC 55312, X56/0, Sample 607-30-1, 53-55 cm, (a) dorsal surface and archeopyle, (b) optical cross-section. 12. Operculodinium sp. of Jan du Chêne (1977), GSC 55313, X53/3, Sample 607-23-4, 49-51 cm, (a) oblique dorsal view, with archeopyle, (b) optical cross-section. 

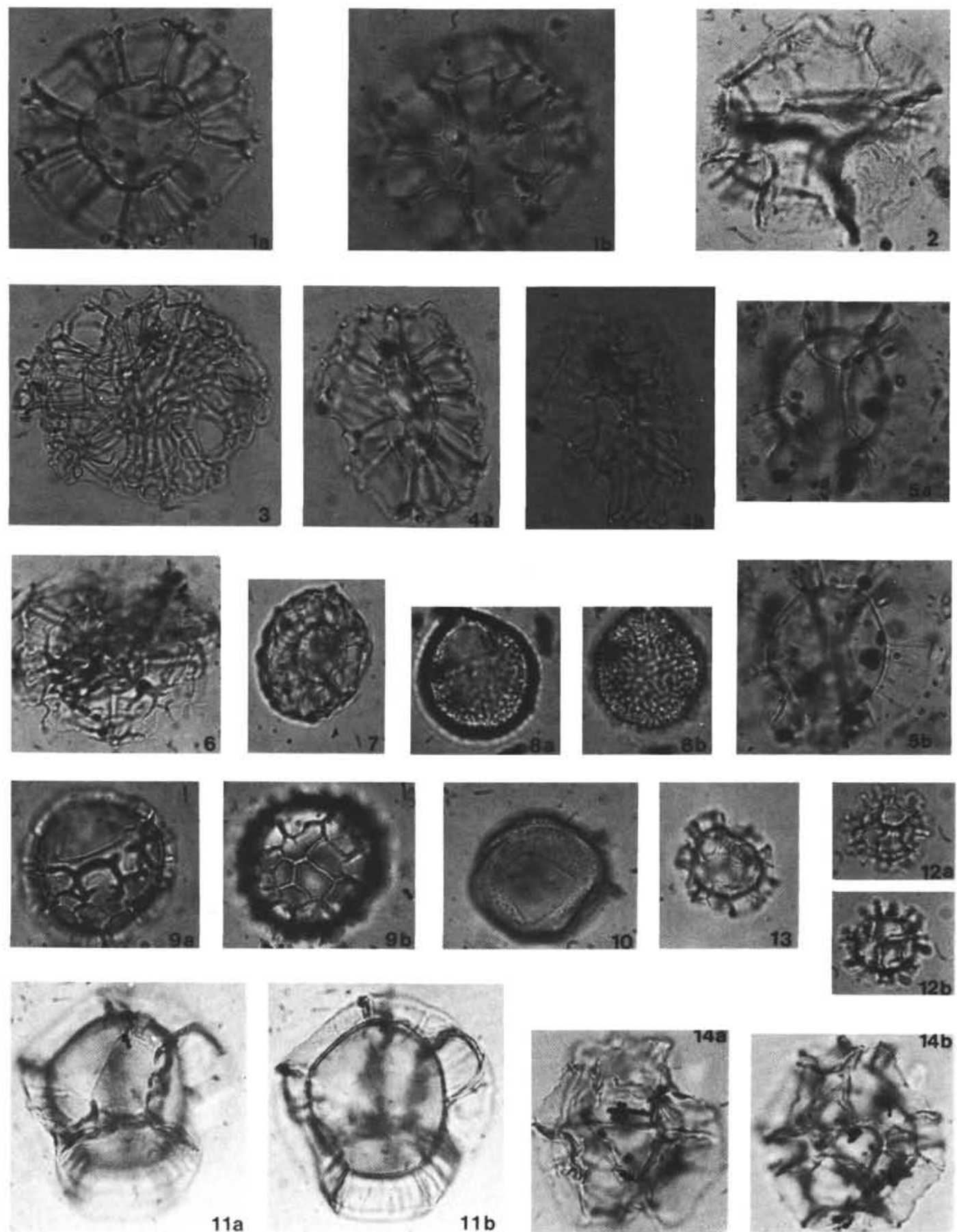

Plate 3. (All magnifications $\times 665$; photographs taken in normal transmitted light.) 1, 3, 4. Sample 611C-35-3, 48-50 cm, (1) Nematosphaeropsis labyrinthea (Ostenfeld) Reid, 1974, GSC 55315, Z56/1 (a, optical section showing central body with archeopyle outline, and rod-like processes; b, surface focus, showing ribbonlike pairs of trabeculae), (3) Nematosphaeropsis sp. 1, surface view, GSC 55317, Y19/1, (4) Nematosphaeropsis oblonga $\mathrm{n}$. sp., GSC 55318, Z55/1 (a, optical section; b, surface of periphragm, showing paired trabeculae). 2. ?Impagidinium pallidum Bujak, 1984, dorsal view, GSC 55316, J52/1, Sample 611C-44-5, 47-49 cm. 5. Impagidinium velorum Bujak, 1984, GSC 55319, H23/1, Sample 607-11-1A, 49-51 cm, (a) dorsal surface and archeopyle outline, (b) optical section showing wide membranous septa supported by thin rod-like processes. 6. ?Cannosphaeropsis sp. 1, surface focus, with spatulate process ending illustrated at bottom right, GSC 55320, Y54/4, Sample $611 C-44-5,47-49 \mathrm{~cm}$. 7. ?Nematosphaeropsis aquaeducta Piasecki, 1980, optical section, GSC 55321, W23/0, Sample 611C-26-2, 48-50 cm. 8, 10. Tectatodinium simplex (Harland) Edwards, 1984, (8) GSC 55322, Y30/2, Sample 611C-33-1, 48-5 cm (a, dorsal surface and archeopyle; b, ventral surface), (10) GSC 55324, Sample 611C-44-5, 47-49 cm, oblique dorsal view showing archeopyle outline. 9. Corrudinium harlandii Matsuoka, 1983, GSC 55323, P15/3, Sample 611C-23-2, 38-40 cm, (a) oblique dorsal view, with part of archeopyle in focus, (b) ventral view. 11. Nematosphaeropsis aquaeducta Piasecki, 1980, GSC 55325, X54/4, Sample 607A-24-1, 49-51 cm, (a) oblique dorsal view with archeopyle at upper left, (b) optical section showing arch-like processes. 12, 13. Labyrinthodinium truncatum Piasecki, 1980, (12) GSC 55326, U31/4, Sample 611C-47-6, 38-40 cm (a, dorsal view showing apical archeopyle; b, ventral surface), (13) GSC 55327, U31/4, Sample 611C-47-6, 38-40 cm, optical section. 14. GSC 55342, Z50/2, Sample $611-35-3,48-50 \mathrm{~cm}$ (a, oblique dorsal view, with irregular outline of apical archeopyle at top center; b, optical section showing unbranched processes). 

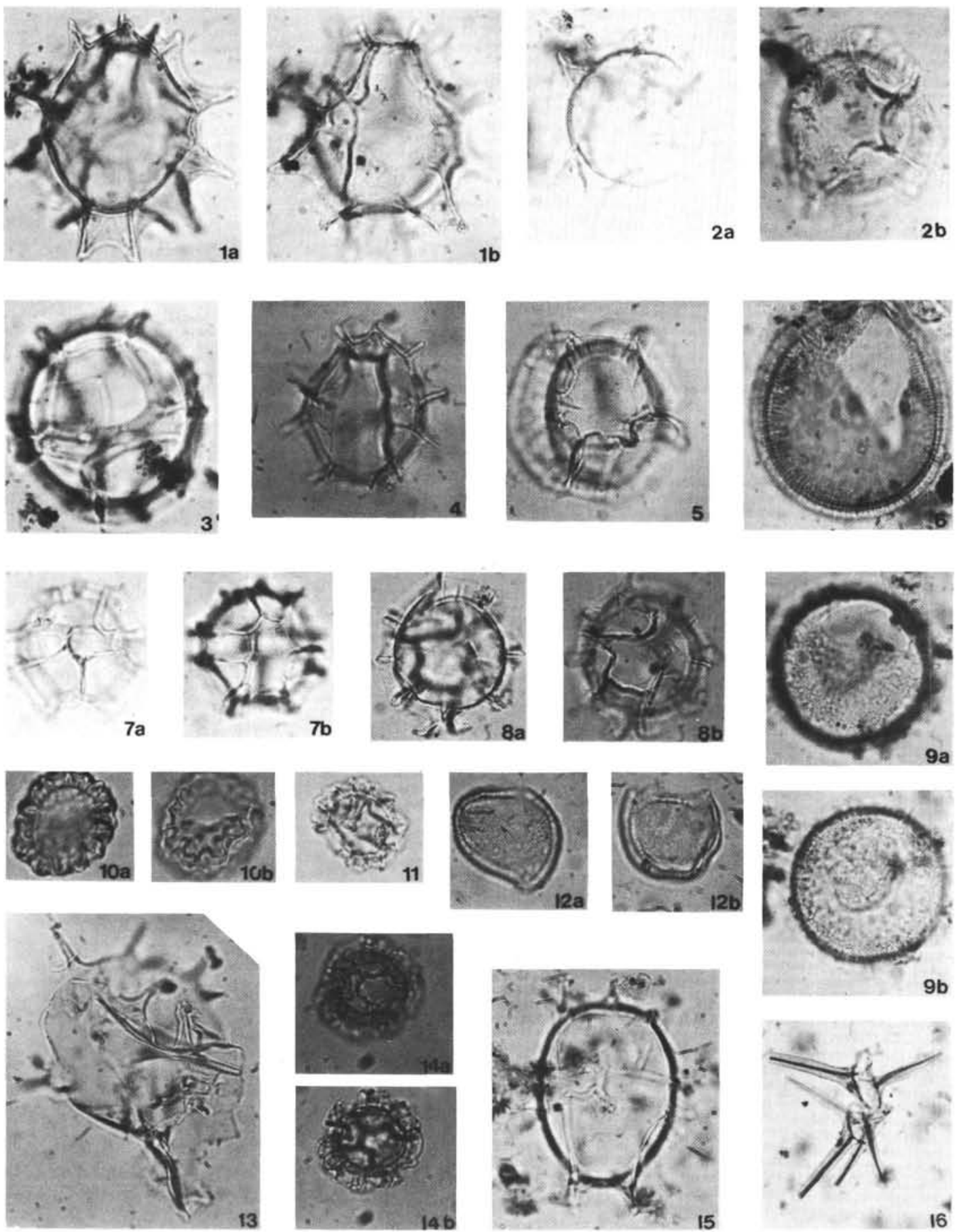

Plate 4. (Magnification $\times 665$, except Fig. 16, which is $\times 285$; all figures photographed in normal transmitted light.) 1, 3, 9. Sample 607-23-6, 49-51 cm, (1) Impagidinium aliferum n. sp., GSC 55329, K28/1 (a, optical cross-section; b, ventral surface, showing wide parasulcus and tubular epitractal process), (3) Impagidinium patulum (Wall) Stover and Evitt, 1978, GSC 55331, U7/0, showing archeopyle with operculum still attached, (9) ?Operculodinium sp. 1, GSC 55337, D54/1 (a, oblique dorsal view of archeopyle; b, optical cross-section, showing operculum apparently still attached). 2. Impagidinium japonicum Matsuoka 1983, GSC 55330, V16/2, Sample 607-9-3, 49-51 cm, (a) optical cross-section, (b) dorsal surface. 4. Impagidinium aculeatum (Wall) Lentin and Williams, 1981, ventral surface, GSC 55332, X27/1, Sample 607-2-5, 49-51 cm. 5, 13. Sample 607-1-4, 49-51 cm, (5) Impagidinium strialatum (Wall) Stover and Evitt, 1978, dorsal surface, GSC 55333, Z43/1, (13) Spiniferites splendidus Harland, 1979, olique dorsal view, GSC 55341, E17/2. 6. Filisphaera filifera Bujak, 1984, dorsal surface, oblique orientation, GSC 55334, R51/3, Sample 607-12-3, 49-51 cm. 7. Impagidinium sp. A, GSC 55335, H38/1, Sample 607-22-2, 49-51 cm, (a) dorsal surface, (b) ventral surface. 8. Impagidinium sp. B., GSC 55336, L44/0, Sample 607-30-1, 53-55 cm, (a) optical cross-section, (b) ?dorsal surface. 10,11. Hystrichokolpoma sp. 1, (10) GSC 55338, Q11/2, Sample 611C-30-5, 48-50 cm (a, optical cross-section; b, archeopyle view), (11) GSC 55339, Q44/2, Sample 611C-30-5, 48-50 cm, ?ventral surface. 12. Pyxidiella sp. 1, (a) oblique view with archeopyle, GSC 55340, T43/0, Sample 611-35-3, 48-50 cm., (b) surface focus, GSC 55365, T32/3, Sample 611-35-3, 48-50 cm. 14. Labyrinthodinium truncatum Piasecki, 1980, GSC 55328, N46/8, Sample 611C-24-6, 48-50 cm, (a) surface view, (b) optical cross-section. 15. Spiniferites elongatus Reid, 1974, dorsal view, GSC 55343, R47/0, Sample 607-1-3, 49-51 cm. 16. Incertae sedis sp. I of Edwards, 1984, specimen damaged, with epitract weakly attached to hypotract, GSC 55344, M48/3, Sample 607-19-6, 49-51 cm. 

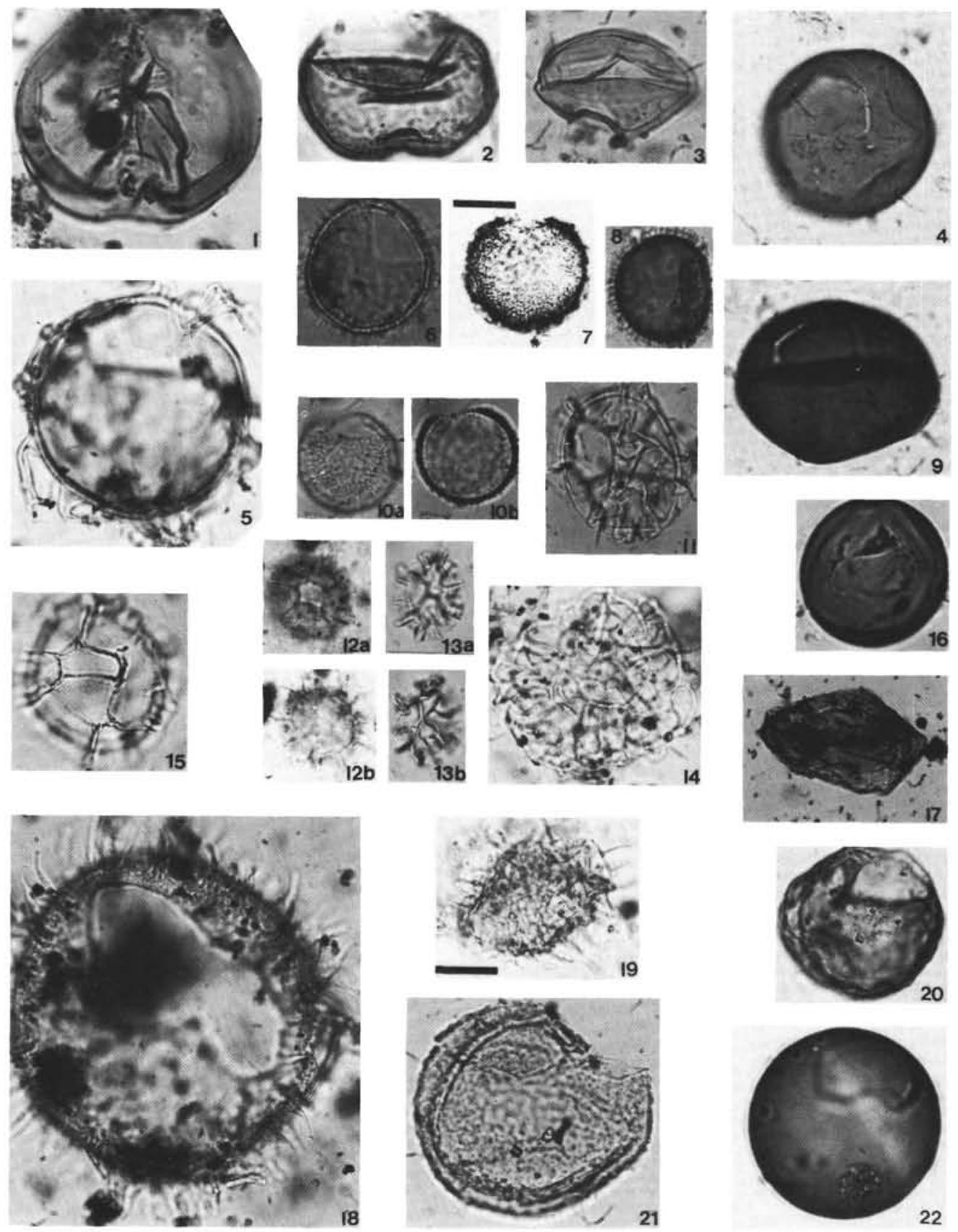

Plate 5. (Magnification $\times 623$, except Fig. 7, which is $\times 267$, and Figs. 6 and 16, where the bar scale is $10 \mu$ m; all figures photographed in normal transmitted light.) 1. Selenopemphix nephroides Benedek, 1972, emend. Bujak, 1980. apical view, GSC 55345, Z20/3, Sample 607-2-6, 49$51 \mathrm{~cm} .2,4,8,9,16,20,22$. Sample 607-1-1, 49-51 cm, (2) Votadinium calvum Reid, 1977, ?ventral view, GSC 55346, E25/2, (4, 9) Brigantedinium simplex Reid, 1974. (4, oblique dorsal view, with archeopyle outline, GSC 55348, K23/0; 9, dorsal surface, archeopyle still attached, GSC 55352, K37/3), (8) Multispinula minuta Harland and Reid in Harland et al., 1980, optical section, with surface of fold on right, GSC 55368, G25/2. (16) Brigantedinium auranteum Reid, 1974, oblique dorsal view, with irregular archeopyle outline, GSC 55350, M48/4, (20) Brigantedinium sp., dorsal view, GSC 55359, O33/1, (22) Brigantedinium cariacoense Reid, 1974, dorsal view with archeopyle still attached, GSC 55349, E49/1. 3. Zygabikodinium lenticulatum Wall and Dale, 1968, emend. Bujak and Davies, 1983, dorsal view, GSC 55347, Y27/0, Sample 611-25, 48-50 cm. 5. Spiniferites sp., optical section, GSC 55353, V49/0, Sample 607A-26-3, 49-51 cm. 6. Operculodinium crassum Harland, 1979, lateral view with archeopyle upper right, GSC 55367, F7/2, Sample 611C-26-2, 48-50 cm. 7. Leiosphaera sp. A of Mudie and Short (1985), GSC 55351; Slide PJM 78-029-24G, 0-1 cm; Sample 78-029-24G, 0-1 cm, from Recent sediment, Baffin Bay. 10, 11. Sample 607-5-6, 49-51 cm, (10) Glenodinium sp. A, GSC 55354, V22/4, Sample 607-5-6, 49-51 cm (a, surface focus; b, optical section), (11) Piperodinium perplexum Mudie, in Scott et al., 1984, surface view, GSC 55355, U55/4. 12-13. Impletosphaeridium spp., (12) GSC 55356, R33/4, Sample 611C20-1, 81-83 cm (a, archeopyle view; b, optical cross-section), (13) GSC 55357, Q51/4, Sample 607-12-3, 49-51 cm (a, optical section; b, surface ornamentation). 14. ?Cannosphaeropsis sp. 1, optical section, GSC 55358, T54/0, Sample 611C-41-5, 92-94 cm. 15. Impagidinium multiplexum (Wall and Dale) Lentin and Williams, 1981, oblique ventral view, GSC 55364, N33/3, Sample 611-7-1, 48-50 cm. 17. Tuberculodinium vancampoae (Rossignol) Wall, 1967, GSC 55362, V43/2, Sample 611C-47-5, 48-50 cm. 18. Lingulodinium machaerophorum (Deflandre and Cookson) Wall, 1967, dorsal view, GSC 55360, L45/4, Sample 607-27-3, 49-51 cm. 19. Multispinula sp. A, optical section, GSC 55361 Slide PJM 70-090B, Sample 70-090B, grab sample from Lancaster Sound, Baffin Bay. 21. ?Palaeostomocystis sp. of Baltes (1971), surface view, with outline of apical archeopyle, GSC 55363, Y43/4, Sample $611 \mathrm{C}-45-1,79-81 \mathrm{~cm}$. 